University of Louisville

ThinkIR: The University of Louisville's Institutional Repository

Electronic Theses and Dissertations

$5-2014$

\title{
Inflammation-resolving lipid mediators promote revascularization to enhance wound healing.
}

Michael Jingyuan Zhang

University of Louisville

Follow this and additional works at: https://ir.library.louisville.edu/etd

Part of the Biophysics Commons, and the Physiology Commons

\section{Recommended Citation}

Zhang, Michael Jingyuan, "Inflammation-resolving lipid mediators promote revascularization to enhance wound healing." (2014). Electronic Theses and Dissertations. Paper 1643.

https://doi.org/10.18297/etd/1643

This Doctoral Dissertation is brought to you for free and open access by ThinkIR: The University of Louisville's Institutional Repository. It has been accepted for inclusion in Electronic Theses and Dissertations by an authorized administrator of ThinkIR: The University of Louisville's Institutional Repository. This title appears here courtesy of the author, who has retained all other copyrights. For more information, please contact thinkir@louisville.edu. 


\title{
INFLAMMATION-RESOLVING LIPID MEDIATORS PROMOTE REVASCULARIZATION TO ENHANCE WOUND HEALING
}

\author{
By \\ Michael Jingyuan Zhang \\ B.S., University of California, Los Angeles, 2008
}

\begin{abstract}
A Dissertation
Submitted to the Faculty of the University of Louisville School of Medicine in Partial Fulfillment of the Requirements for the Degree of

Doctor of Philosophy

Department of Physiology and Biophysics

University of Louisville

Louisville, Kentucky
\end{abstract}

May 2014 



\title{
INFLAMMATION-RESOLVING LIPID MEDIATORS PROMOTE REVASCULARIZATION TO ENHANCE WOUND HEALING
}

\author{
By \\ Michael Jingyuan Zhang \\ B.S., University of California, Los Angeles, 2008 \\ A Dissertation Approved on \\ April 16, 2014 \\ By the following Dissertation Committee:
}

Advisor: Aruni Bhatnagar, Ph.D.

Co-Advisor: Matthew Spite, Ph.D.

Daniel Conklin, Ph.D.

Dale Schuschke, Ph.D.

Stanley D'Souza, Ph.D.

William Wead, Ph.D. 


\section{ACKNOWLEDGEMENTS}

I have had the privilege of guidance from incredible mentors throughout my training. In chronological order, they are my parents, Peipei Ping, Thomas Vondriska, Kym Faull, Aruni Bhatnagar and Matthew Spite. My future career will be built upon the comprehensive foundation they have helped me established. I will be always grateful for their wisdom, patience, and trust. 


\section{ABSTRACT \\ INFLAMMATION-RESOLVING LIPID MEDIATORS PROMOTE REVASCULARIZATION TO ENHANCE WOUND HEALING}

Michael J. Zhang

May 10, 2014

Wound healing is a highly concerted cellular process that begins with inflammation and proceeds to resolution to revascularize the site of injury. Although inflammation is essential to revascularization during wound healing, it is now recognized that resolution is an active process that is equally important. Other investigations have implicated a beneficial effect of resolving inflammation and promoting resolution in the remission of inflammatory pathologies. Recently investigations have yielded a novel class of $\omega-3$ fatty acid derived lipid mediators, biosynthesized by leukocytes, which are capable of resolving inflammation and promoting resolution. We therefore hypothesized that these leukocyte-derived pro-resolving lipid mediators can promote revascularization to enhance wound healing. In the following studies, we provide evidence supporting this hypothesis: 1 ) that the $\omega-3$ fatty acid derived pro-resolution lipid mediator Resolvin D2 enhances perfusion recovery of ischemic tissue 2) that inflammatory monocytes, which increase during revascularization, synthesize Resolvin D2 through the 12/15-lipoxygenase pathway 3) and that resolvin D2 does not stimulate angiogenesis, but stimulates arteriogenesis through the promotion of endothelial cell migration. These results suggest that Resolvin D2 can enhance revascularization and may be an effective therapeutic agent. 


\section{TABLE OF CONTENTS}

ACKNOWLEDGEMENTS .....................................................................................................................II

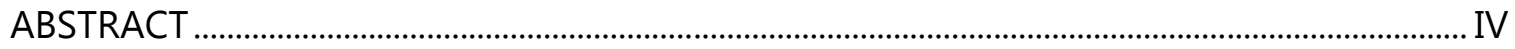

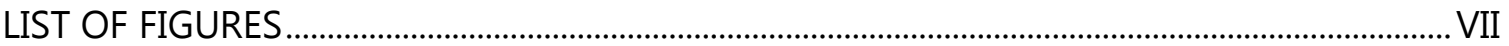

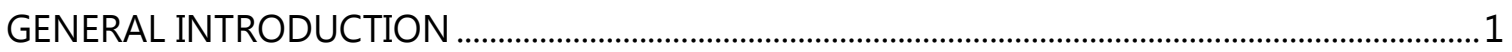

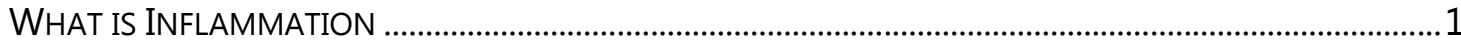

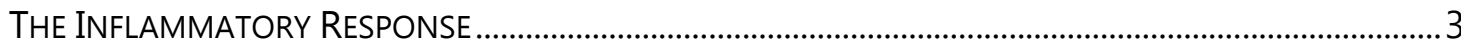

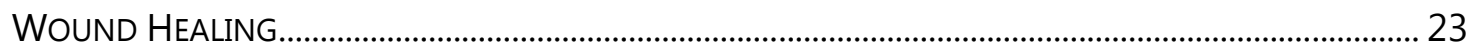

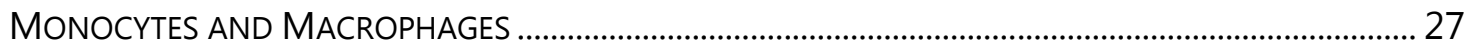

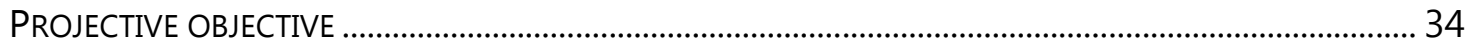

RESOLVIN D2 ENHANCES POST-OCCLUSION REVASCULARIZATION ....................................... 37

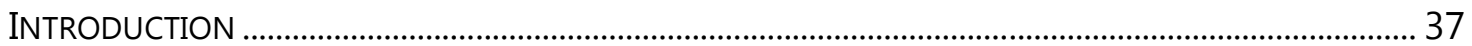

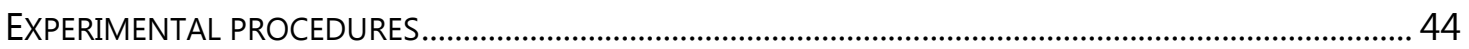

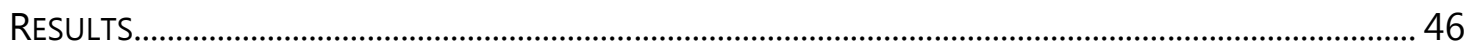

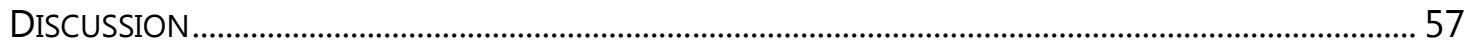

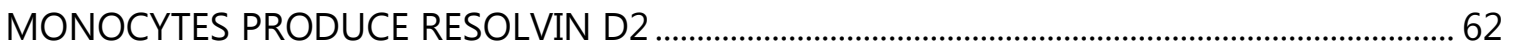

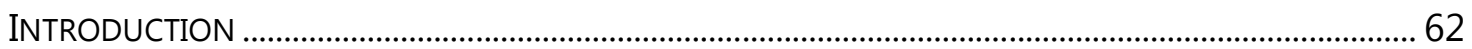

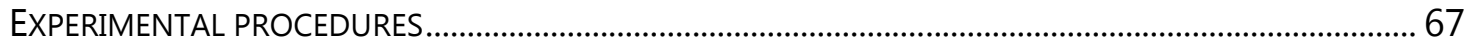


DISCUSSION

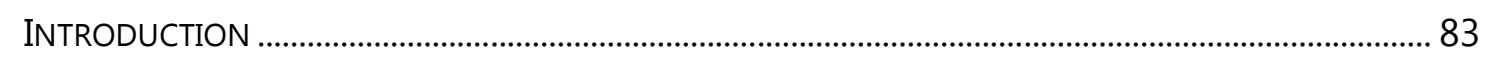

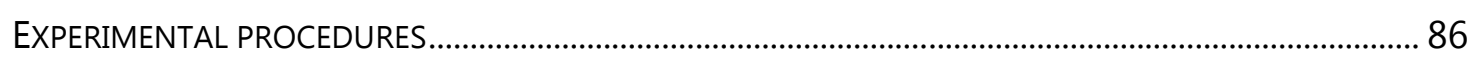

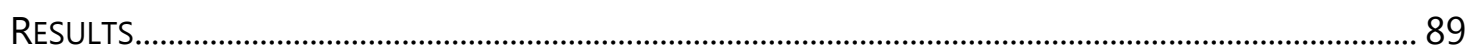

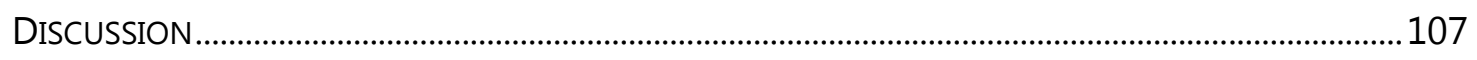

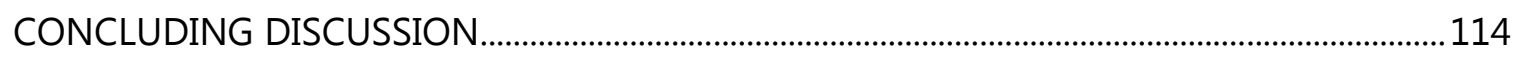

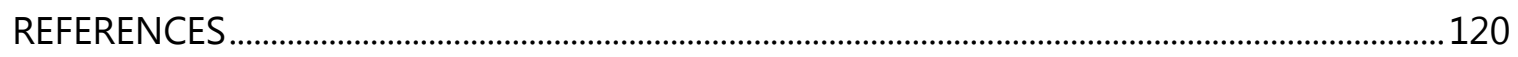

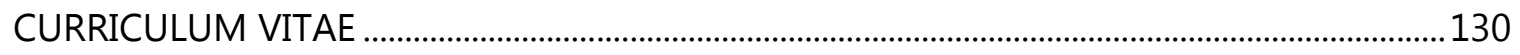




\section{LIST OF FIGURES}

FIGURE

PAGE

1. Laser doppler perfusion imaging (LDPI) of mouse hind limb .50

2. MicroCT of Microfil-casted limbs, severe HLI model .52

3. Whole mount and microCT images of Microfil-casted limbs .54

4. Histology of hamstring muscles, severe model of hind limb ischemia .56

5. 12/15-lipoxygenase biosynthesis products during revascularization .73

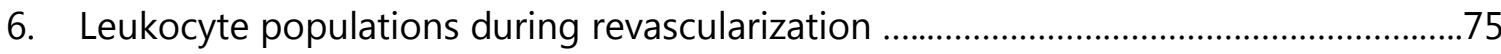

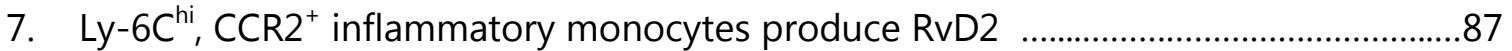

8. Leukocytes numbers in ischemic muscles are not altered by RvD2 ...........................94

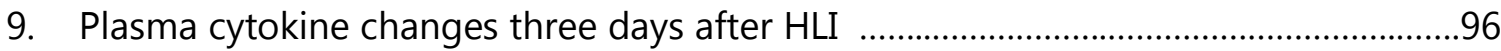

10. mRNA expression changes in hamstring muscles $3 \mathrm{~d}$ after HLI ....................................98

11. mRNA changes in RvD2-stimulated peritoneal macrophages ...................................100

12. RvD2 stimulates endothelial migration but not proliferation ......................................102

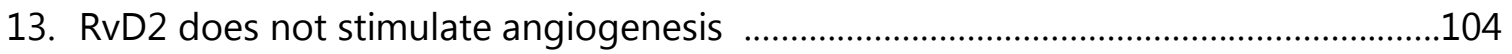

14. RvD2 stimulates migration through eNOS and Rac1 …...........................................116 


\section{CHAPTER I}

\section{GENERAL INTRODUCTION}

\section{What is Inflammation}

\section{Historical perspective on inflammation}

One of the defining characteristics of biological systems is the capacity to maintain oneself through self-repair. Unicellular organisms, such as bacteria, are capable of repairing damaged DNA ${ }^{1}$. Multicellular invertebrates such as annelids can regenerate entire body segments ${ }^{2}$. Important agronomic plants can inosculate their vasculature following graftage ${ }^{3}$. Humans can repair skin tissue after traumatic abrasions ${ }^{4}$. All of these reparative processes require tightly orchestrated molecular and cellular actions. While the amoeba is confined to autologous repair mechanisms ${ }^{5}$, multicellular organisms of greater evolutionary complexity have the luxury of specialized cells that concentrate on ameliorating injury.

A common injury that afflicts multicellular organisms, such as humans, is bacterial infection. The body's reparative response to this attack is broadly termed inflammation. In the Smith Papyrus, a scroll written around 1650 BCE in Egypt, a hieroglyphic word used in connection with infected wounds contained a flaming brazier, to convey the idea of a "hot thing" ${ }^{\prime 6}$. Later in Greek medicine, infected wounds were described with the word phlegmoné, roughly translated as "the burning thing". Finally the Roman, Celsus, established the precise clinical definition rubor et tumor cum calore et dolore, "redness and swelling with heat and pain" as the cardinal signs of inflammation ${ }^{7}$. Centuries later, Julius Cohnheim published a key manuscript describing in vivo evidence of these cardinal signs in frog mesentery and tongue ${ }^{6}$. He exposed this tissue in the 
living frog under a microscope, causing irritation. This inflamed state immediately produced arteriolar dilation and accelerated blood flow in the entire vascular network. Minutes later, blood flow slowed and some white blood cells began to adhere to the venule walls. Then, slowly, the adhered cells began to squeeze through venule walls and occupy extravascular space. Finally, blood flow halted in some venules that appeared collapsed with tight aggregations of red blood cells. Cohnheim made the inference that plasma had escaped the venule into the tissue due to increased vascular permeability. He had also made the first explanation of the cardinal signs of inflammation: vasodilation caused rubor; increased blood flow generated calore; transudation of white blood cells and exudation of plasma produced tumor; and surely the frog was not spared of dolore. This series of observations and insights remains a foundational description of inflammation: the highly concerted and multi-modal sequence of blood-borne and tissueresident responses elicited by endogenous tissue damage or exogenous pathogenic assault.

The fundamental role of this complex response sequence is to protect the host by limiting tissue damage and then facilitating tissue repair ${ }^{7}$. Tissue damage encompasses bacterial infections, but also includes other microbial pathogens, traumatic wounds, ischemic damage and even autoimmune attacks. These insults must be swiftly contained to prevent festering of the damage. Tissue-resident macrophages are capable of phagocytosing microbial pathogens and thus trapping them in digestive lysosomes ${ }^{8}$. Infiltrating neutrophils will palisade around large clusters of assailants to form quarantining granulomas ${ }^{9}$. Once the damage is contained, inflammation then transitions to prevent future damage and regenerate the injured tissue ${ }^{7}$. Lymphocytes are recruited to recognize, then memorize, the offending pathogens and patrol the host for future attacks. Macrophages complete the phagocytic cleanup of the necrotic or apoptotic detritus, and also digest the extracellular matrix to create virgin space for regenerating cells. Tissue homeostasis can then be restored with the arrival of endothelial and smooth muscle 
cells to rebuild vasculature, fibroblasts to reconstruct the collagen matrix, and the differentiation of stem cells to specialized parenchymal cells. The renowned pathologist Rudolf Virchow recognized a fifth cardinal sign of inflammation, functio laesa, disturbed function ${ }^{10}$. While this is certainly true during the inflammatory response, the desired endpoint of inflammation is complete elimination of noxious stimuli and the reestablishment of tissue function.

This ability to self-repair is what differentiates biological from mechanical systems, as a result they are infinitely more adaptable and enduring. The caveat of this longevity is competition among biological systems (animals vs. plants vs. microbes) for scarce resources. However, one outcome of this struggle appears to be a permanent alliance: a presumed inflammatory reaction in a eukaryote resulted in the phagocytosis of a prokaryote that symbiotically specialized into mitochondria $^{11}$. Humans are the aggregates of successive alliances between a highly specific social order of cells ${ }^{12}$. Inflammation is one such alliance that empowers humans to overcome external insults and repair internal wounds.

\section{The Inflammatory Response}

Given the remarkable capabilities of the human inflammatory response, it is the subject of intensive scientific investigation. Since Cohnheim's significant observations, the organular, cellular and molecular timecourse of inflammation has been comprehensively documented in both humans and model organisms such as mus musculus. The timecourse first begins with the triggering of inflammation; this is precipitated by exogenous microbes or endogenous necrosis ${ }^{11}$. The onset of inflammation is then rapid, typically minutes, and then escalates over the course of hours. Waves of infiltrating leukocytes and perfused protein and lipid mediators from the blood work in concert with resident cells to cordon, then neutralize, the source of injury. This phase of inflammation is variable in length corresponding to the severity of damage. Eventually the stimuli 
of inflammation subside and the escalated response shifts to resolution of inflammation phase. Now a new group of leukocytes and mediators prevail to quench the initial inflammatory escalate and debride the damage site. This cleansing of damage then facilitates the final proliferation and remodelling phase where vascular, structural and parenchymal cells repopulate. Inflammation thus abates with the return of the injured tissue to functional homeostasis.

Initiation of inflammation

Exogenous pathogens can easily tilt the delicate poise of homeostasis to trigger inflammation through stimulation of cellular pattern recognition receptors. Viruses, bacteria, fungi and parasites all produce virulence factors designed to invade a host, colonize a niche and then reproduce progeny. The inflammatory response is able to neutralize these factors with an arsenal of molecular sensors. One large class of sensors is the pattern recognition receptors (PRR) ${ }^{7}$. These receptors bind to unique moieties of pathogens that are not present in the host. Toll-like receptors, named after the drosophila gene Toll, are a large sub-class of PRR with a common receptor domain mostly on leukocyte cell surfaces that recognize a wide range of bacterial and fungal lipoproteins, glycolipids, and unmethylated DNA sections. C-type lectin receptors are another large class of PRRs existing predominantly on dendritic cells and macrophages that bind primarily to microbial carbohydrates in a calcium dependent manner. Ligands for these receptors have been discovered on HIV virus, mycobacteria, candida and leishmania. Nucleotide-binding oligomerization domain receptors (NOD-like receptors) are cytosolic PPRs that recognize various constituents of the bacterial peptidoglycan layer. Retinoic acid-inducible gene 1-like receptors (RIG-I-like receptors) also exist in the cytoplasm and detect double-stranded RNA from viruses. All of these pattern recognition receptors are able to identify their microbial targets and then interact with host genes and proteins to escalate the inflammatory response. Another large class of 
exogenous pathogen sensors is the immunoglobulins that circulate in the blood or anchor to lymphocyte cell surfaces. Through exceedingly diverse VDJ genetic recombination and somatic hypermutation, $\mathrm{B}$ and $\mathrm{T}$ lymphocytes produce immunoglobulins that can recognize any antigen ${ }^{13}$. Although they are produced initially in the later periods of inflammation, the immunoglobulins that bind antigen with the highest affinity are retained. They then patrol the host to sense future attacks by the same pathogen. Once the returning pathogen is recognized, immunoglobulins will attach en masse and signal immune cells or the complement system to initiate inflammation ${ }^{14}$. This latter system is also able to sense pathogens independently. While the classical pathway of complement is activated by immunoglobulins, a lectin and alternative pathway can also bind to non-host moieties like pattern recognition receptors and then stimulate inflammation. The final group of pathogen sensors are inadvertent and serve a primary purpose in the host body but can be commandeered by pathogens for their own use. One example is the staphylococcal enterotoxin B and its interaction with host major histocompatibility complex class II (MHC II) receptor $^{15}$. MHC II receptors are normally present on antigen-presenting cells and B-lymphocytes, and interact with T-lymphocytes to select high affinity immunoglobulins. Enterotoxin B binds to the MHC II receptor directly, and stimulates a violent and non-specific inflammatory response by the antigen-presenting cells. This resulting torrent of inflammation presents clinically as toxic shock syndrome. Fortunately most sensors of exogenous pathogens are highly specific and only stimulate tightly controlled inflammatory responses.

Not all signals of inflammation derive from exogenous pathogens; endogenous injuries must be rapidly identified as well. In the case of traumatic wounds, much of the injury is to endogenous tissue and vasculature. Cells in these wounds do not apoptose in an inert fashion but instead rupture necrotically to spew their protein and nucleic acid contents into the tissue or blood $^{7}$. Some of these molecules directly stimulate inflammation once released. For example, high 
mobility group protein 1 (HMGB1) is an abundantly expressed nucleosome protein ${ }^{16}$. When it is discharged from the cell during necrosis, it can directly stimulate leukocytes to generate inflammatory mediators. Uric acid is a ubiquitous metabolite of cellular purine recycling. When it is unintentionally released outside the cell, it combines with higher levels of free sodium to form monosodium urate microcrystals. These crystals are potent stimulators of inflammation, evidenced during an episode of gout. Many more highly expressed intracellular proteins are directly inflammatory; these include the heat shock proteins, galectins, S100 proteins, and mitochondrial $\mathrm{N}$-formyl peptides ${ }^{17}$. However since cells produce an extensive collection of proteins, inflammatory signals from necrotic cells are still being actively catalogued. Another common injury occurring in tissue is hypoxia. This occurs when uncompensated coagulopathies cause ischemia and deprive tissues of oxygen. The master sensor Hypoxia inducible factor 1 (HIF1) is constitutively expressed, but also constantly prolyl hydroxylated in an oxygen-dependent manner that targets the active alpha subunit of HIF-1 into the proteasomal degradation pathway ${ }^{18}$. Upon hypoxia, prolyl hydroxylation is inhibited and the alpha subunit of HIF-1 is stabilized. It then translocates to the nucleus to stimulate the nuclear factor $\mathrm{kB}(\mathrm{NF}-\mathrm{kB})$ family inflammatory transcription factors. Because tissue damage is a frequent sequelae of tissue hypoxia, constant turnover of HIF-1 allows cells to rapidly respond to oxygen deprivation and trigger repair. Overall, the consequences of internal tissue damage can be just as noxious as external pathogenic invasion, thus animals have evolved numerous sensors to identify a disturbance of homeostasis and initiate an inflammatory response.

\section{Escalation of inflammation}

Once inflammation is triggered, the reaction is escalated with the participation of multiple modalities. These include tissue responses, cellular responses and secreted mediator responses; 
some are indiscriminate, others are highly specific. In general the rapid onset of inflammation, acute inflammation, proceeds in a broadly tri-phasic sequence: vascular caliber is altered to increase blood flow; structural changes in the vasculature allow leukocytes and secreted mediators to leave the circulation; extravasation of these cells and diffusion of these molecules into the locus of injury to contain the damage ${ }^{7}$.

The existence of vasculature, with its circulating leukocytes and secreted mediators, allows the inflammatory response to target any site where they may be required ${ }^{6}$. When the vessels perfusing the area of damage are activated by sensors of inflammation, the immediate reaction is sometimes a vasospasm lasting mere seconds, but always followed by prolonged vasodilation $^{7}$. First the arterioles expand and then the downstream capillary beds increase blood flow. This increased perfusion is caused by separation of endothelial cells from stimulation by histamine, a nitrogenous compound produced and stored in granules in mast cells and basophils, and vascular smooth muscle relaxation from nitric oxide, a volatile gas generated by nitric oxide synthase in endothelial cells and leukocytes. Along with vasodilation there is an increase in vascular permeability from the activation of endothelial cells. Secreted mediators such as histamine, bradykinin, leukotrienes, and neuropeptide substance P cause an immediate transient contraction of endothelial cells, thus opening their tight junctions and allowing blood contents to enter the extracellular space ${ }^{7}$. Direct physical injury to endothelial cells will result in cell necrosis and detachment, also forming more vascular leakage sites. This combination of dilation and permeability results in fluid loss to the extracellular space and a retardation of blood flow ${ }^{7}$. Erythrocytes accumulate as a result, increasing the local viscosity of blood in already dilated vessels, and producing a local rubor ${ }^{7}$. More importantly, leukocytes, platelets and secreted mediators all accumulate as well.

The predominant first-responding leukocyte in any inflammatory event is the 
neutrophil ${ }^{14}$. These cells arise from hematopoetic stem cells in the bone marrow and bear unusually lobular and segmented nuclei. As stasis develops in dilated and permeablized vasculature, neutrophils begin to migrate away from the center of blood vessels towards the margins near the endothelial surface. Specific adhesion molecules, such as selectins and integrins, produced by both neutrophils and activated endothelial cells allow neutrophils to slow, roll, and then arrest on the endothelial surface. Neutrophils produce L-selectin and endothelial cells produce E-selectin and $\mathrm{P}$-selectin to facilitate the slowing and rolling process ${ }^{19}$. These selectins are normally stored in intracellular granules, but redistribute to the cell surface following histamine and other secreted mediators to allow binding of both cell types to various sialylated oligosaccharide-linked mucin-like glycoprotein backbones. Subsequently, neutrophils produce integrin $\alpha 4 \beta 1$ and integrin $\alpha L \beta 2$, while endothelial cells correspondingly produce vascular cell adhesion molecule 1 (VCAM-1) and intercellular adhesion molecule-1 (ICAM-1) to consolidate the neutrophil-endothelium adhesion ${ }^{19}$. Once adhered, the neutrophil rearranges its cytoskeleton and spreads out on the endothelium towards secreted mediator gradients that direct it past the endothelial layer, through the basement membrane and into the damaged tissue.

Once infiltrated into the inflamed site, neutrophils begin the process of wound debridement. They surround loci of invading pathogens or necrotic tissue by palisading and begin the complex process of phagocytosis ${ }^{7}$. This involves three sequential stages: recognition and attachment to tissue injury agents; engulfment of these agents into a phagolysosome; and finally the digestion or degradation of ingested contents. The three primary recognition receptors involved in the process are mannose, scavenger, and opsonin receptors. Mannose receptors bind to glycoproteins and glycolipids found on microbial cell walls but not mammalian membranes ${ }^{20}$. Scavenger receptors were originally characterized as gatherers of oxidized or acetylated lowdensity lipoprotein particles incapable of interaction with the normal LDL receptor. However 
scavenger receptors have now been found to also bind a variety of pathogenic microbes ${ }^{21}$. Fc receptors are the largest group of opsonin receptors ${ }^{22}$. They are members of the immunoglobulin superfamily and recognize various Fc regions of different antibody classes. After circulating antibodies bind to pathogenic targets, leukocytes attach via Fc receptors, or mannose and scavenger receptors independently, and begin the engulfment process ${ }^{7}$. Pseudopodic extensions of leukocyte cytoplasm, formed by small guanine triphosphate hydrolases (GTPases) stimulated actin filament polymerization, surround the microbe and pinch off to form an intracellular phagocytic vesicle (phagosome). This phagosome then fuses with a digestion enzyme-containing lysosome and becomes a phagolysosome. Next, the multiprotein NADPH oxidase complex translocates to the phagolysosomal membrane and releases reactive oxygen species within the isolated phagolysosome. This is not sufficient to neutralize pathogens completely however. Myeloperoxidase (MPO) contained within neutrophil granules then combine with reactive oxygen species, in combination with intracellular chloride, to form hypochlorite. This is the most potent antimicrobial agent due its non-specific covalent halogenation and oxidation of microbial cell constituents. The destroyed microbes are either fully digested by the fused lysosomal enzymes or released into the interstitium via the plasma membrane. Dead host cells can be digested in this same process, however their recognition is, by necessity, different from pathogens. These dead cells are identified by phagocytes through a set of colloquially termed find me and eat me signals $^{23}$. Find me signals include fractalkine, lysophosphatidylcholines, sphingosine-1-phosphate and P2Y-binding nucleotides. Eat me signals include phosphatidylserine, oxidized LDL, calreticulin and altered cell surface glycoproteins. Cells that produce these signals can be damaged parenchymal cells and vascular cells, but also includes recently infiltrated neutrophils that have already phagocytosed microbes or debris and are apoptosing themselves. Finally, concomitant with the engulfment of microbes and cells is the release of secreted mediators of inflammation 
that further propagate inflammation by recruiting more leukocytes.

The secreted mediators of inflammation are a hugely diverse group of molecules that are produced in a highly coordinated manner that largely determines the onset, magnitude and duration of the reaction. Inflammation mediators are produced by cells or assembled from plasma proteins ${ }^{7}$. Cell-derived mediators are either stockpiled a priori in intracellular granules for rapid secretion (e.g., histamine in mast cell granules) or generated specifically in response to inflammatory stimuli (e.g., prostaglandins, cytokines). The primary producers of these mediators during inflammation are platelets, neutrophils, monocytes, macrophages, mast cells, endothelial cells, smooth muscle cells and fibroblasts. Plasma-derived mediators, such as the complement system and coagulation system, are released from the liver as inactive zymogens circulating in the blood that must be activated through proteolytic cleavage during inflammation. Both cell and plasma derived mediators are only activated by sensors of inflammation to ensure both a highly specific and timely response to tissue injury. Once activated, mediators cascade by stimulating the release of other mediators to amplify the original sensor response. These mediators affect assortment of cellular targets; they can affect several different types of cells simultaneously or effect different responses in different cells. And finally, once activated, most mediators are then rapidly inactivated through enzymatic degradation (arachidonic acid metabolites), molecular scavenging (reactive oxygen species) or molecular inhibition (antithrombin) to limit the inflammatory response to only what is sufficient to address the injury.

Histamine and serotonin are the two major vasoactive amines that act as secreted mediators of inflammation. Both molecules are preformed and stored in intracellular granules for rapid secretion, typically on the precipice of inflammation. Histamine is produced by both immune cells and cells of the digestive system, but during inflammation, their primary reservoir is the mast cell ${ }^{24}$. Physical trauma or temperature extremes, membrane crosslinking by antibodies, 
C3a and C5a anaphylatoxins of the complement system, neuropeptides, and cytokines and chemokines, can all stimulate mast cell degranulation of histamine. Once released, histamine binds to $\mathrm{H} 1 \mathrm{G}$ protein-coupled receptors on endothelial cells to increase interendothelial gaps and thus more vascular permeability. Meanwhile, serotonin stimulates this same effect from release by platelet degranulation after contact with collagen, thrombin, adenosine diphosphate or certain antibody-antigen complexes ${ }^{25}$. In fact, serotonin can mediate the transformation of the desert locust, Schistocerca, from a benign solitary state to the gregarious phase responsible for sky-darkening and crop-destroying swarms; perhaps there are some fractal analogies in serotonin-signaled processes ${ }^{26}$. While these two amines are often the first initiators of vasodilation due to their preformed nature, many more inflammatory vasodilators are produced.

Nitric oxide (NO) is another nitrogen-containing vasoactive mediator. NO is a volatile and highly soluble gas produced by three separate enzymes; endothelial nitric oxide synthase (eNOS) in endothelial cells; inducible nitric oxide synthase (iNOS) in leukocytes; and neuronal nitric oxide synthase $(\mathrm{nNOS})^{27}$. NO has diverse cellular roles. During bacterial infections, NO in leukocytes serves a microbicidal role by interfering with metal centers and thiols necessary for respiration and DNA replication. Extracellularly, due to the mere-seconds half-life of NO, it only acts in a paracrine manner on neighboring cells and principally through the stimulation of cyclic guanosine monophosphate (cGMP). cGMP then initiates a series of intracellular pathways that stimulate vascular smooth muscle relaxation, platelet aggregation and adhesion reduction, mast cell stabilization and leukocyte infiltration attenuation. Thus NO not only stimulates inflammation, through smooth muscle vasodilation, but also inhibits inflammation, through blood cell inhibition.

Reactive oxygen species (ROS) are another group of volatile secreted mediators of inflammation. They include the superoxide anion, hydrogen peroxide and hydroxyl radicals ${ }^{28}$. Additionally, ROS can combine with NO to form reactive nitrogen species. These ROS are 
generated by the NADPH oxidase system in leukocytes after exposure to microbes, antibody complexes or other secreted inflammatory mediators. The primary objective of ROS generation is to neutralize phagocytosed microbes, but due to their highly diffusive nature, they are also capable of damaging other cells and molecules. Damage to endothelial cells will further increase vascular permeability; inactivation of $\alpha 1$-antitrypsin can cause inadvertent extracellular matrix destruction through unopposed protease activity. However, not all highly diffusive inflammatory mediators are directly cytotoxic.

Lipid derived inflammatory mediators are also able to readily cross cellular membranes to act as soluble mediators of inflammation. The largest and most diverse group is the polyunsaturated fatty acids (PUFA) such as linoleic acid, arachidonic acid, eicosapentaenoic acid, and docosahexaenoic acid ${ }^{14}$. These PUFAs are normally sequestered in esterified form within plasma membrane phospholipids. During inflammation, cellular influx of calcium or other mediators stimulate phospholipase (primarily A2) to liberate the PUFAs from the membrane to be converted by cyclooxygenases into prostaglandins and lipoxygenases into leukotrienes. Cyclooxygenase 2 is induced during inflammation and biosynthesizes prostaglandin lipid mediators $^{14}$. Various lipoxygenase enzymes operate in succession to produce an even larger class of mediators that include leukotrienes and lipoxins ${ }^{29}$. Despite their varying biosynthetic pathways, all lipid mediators interact with $G$ protein-coupled receptors, as agonists or antagonists (or inverse agonists) to direct or influence all phases of inflammation. Because they were discovered first, prostaglandins have been the most extensively characterized. They are biosynthesized by many cell types, including mast cells, neutrophils, macrophages and endothelial cells. The most abundant products generated during inflammation are prostaglandin $E_{2}\left(P_{G} E_{2}\right), P G D_{2}, P G I_{2}$ and thromboxane $A_{2}\left(T x A_{2}\right)^{14}$. Mast cells generate abundant $P G D_{2}$ to stimulate vasodilation, increase post-capillary venule permeability, and enhance neutrophil chemotaxis. $\mathrm{PGI}_{2}$ is also a potent 
vasodilator and it inhibits platelet aggregation. Conversely, $\mathrm{TxA}_{2}$ is synthesized by platelets, and stimulates platelet aggregation and promotes vasoconstriction. $\mathrm{PGE}_{2}$ is the most widely distributed and most abundantly generated cyclooxygenase product, however the diverse actions of $\mathrm{PGE}_{2}$ are still under intense investigation ${ }^{30}$. Like its isomer $\mathrm{PGD}_{2}, \mathrm{PGE}_{2}$ can stimulate mast cell degranulation to cause vasodilation or it can stimulate vasodilation directly through four EP receptors. $\mathrm{PGE}_{2}$ can enhance neutrophil infiltration and promotes activation, maturation and migration of dendritic cells. However, paradoxically, $\mathrm{PGE}_{2}$ can limit the phagocytic capability of alveolar macrophages. It can suppress some of the cytolytic capabilities of natural killer cells. $\mathrm{PGE}_{2}$ can also indirectly propagate inflammatory pain and systemic fever. Finally, $\mathrm{PGE}_{2}$ can stimulate the anti-inflammatory cytokine, IL-10 as well. In short, $\mathrm{PGE}_{2}$ has different effects on different cells, and more recently appreciated, has different effects at different time points during inflammation. The production, targeting and effects of $\mathrm{PGE}_{2}$ are thus highly context dependent. Because eicosanoids readily traverse cell membranes, have minute but highly specific steric differences, and act mostly in a local paracrine mode, there are major technical limitations in monitoring their biosynthesis and movement spatially and in real-time ${ }^{31}$. However, as previously alluded, one major conceptual realization recently has been temporal importance of their biosynthesis ${ }^{32}$. $\mathrm{TXA}_{2}$ and $\mathrm{PGI}_{2}$ are produced primarily during the initiating phases of inflammation. Then eicosanoid production shifts to another class, leukotrienes (described later) to stimulate leukocyte infiltration. The later phases of inflammation, pathogenic and cellular debris cleanup, and parenchymal reestablishment, are influenced by another set of eicosanoids, lipoxins and resolvins. Therefore the context dependent paradoxical actions of $\mathrm{PGE}_{2}$ could be simply the result of temporal synthesis differences. As detection technologies improve for eicosanoids, this temporal factor will become better characterized and understood.

The other major group of eicosanoids is the lipoxygenase enzyme products and they act 
as soluble mediators of inflammation as well ${ }^{33}$. There are many different isoforms of lipoxygenases, resulting in a huge variety of biosynthetic products. The most extensively characterized are the 5-lipoxygenase products leukotriene B4 (LTB4) and cysteine residuecontaining LTC4, LTD4 and LTE4. The cysteinyl leukotrienes are powerful vasoconstrictors that cause bronchospasms, but they also increase vascular permeability and stimulate endothelial cells and many different leukocytes. LTB4 has similar actions and is also an extremely potent neutrophil chemoattractant. It is generated most abundantly after the initial edema phase of inflammation during which neutrophil infiltration is at its peak. Many different types of leukocytes can produce LTB4, including the neutrophil itself. Furthermore, LTB4 production is not always confined to a single cell. In vitro studies of coincubated leukocytes have demonstrated that biosynthetic enzymes of the LTB4 pathway in different cells can operate synergistically to produce additional LTB4 product. Thus in addition to specific temporal generation, eicosanoids are capable of transcellular synthesis. Undoubtedly, much remains to be elucidated in eicosanoid biology.

The final group of cell-derived inflammatory mediators, cytokines and chemokines, is the most diverse, most investigated, most targeted pharmaceutically, and yet remains incompletely understood. The majority of cytokines are involved in inflammation primarily through their utilization by the adaptive immune system are thus not directly involved in the acute inflammatory response ${ }^{7}$. Tumor necrosis factor alpha (TNFa) and interleukin-1 (IL-1), however, are primary mediators of inflammation ${ }^{7}$. In general, TNFa and IL-1 are produced by activated monocytes and macrophages in response to stimulation by bacterial toxins, immune complexes and physical injury. Unlike NO and eicosanoids, the effects of TNFa and IL-1 are both local and systemic. TNFa can affect the hypothalamus, and induce fever and suppress appetite. It can also stimulate neutrophil infiltration and macrophage phagocytosis. Along with IL-1, it can activate the endothelium to increase endothelial adhesion molecule expression, it can increase the 
thrombogenicity of the endothelium, and it can increase the production of other cytokines, chemokines, growth factors, eicosanoids, nitric oxide and matrix remodeling enzymes. Due to their far-reaching inflammatory effects, TNFa and IL-1 are both targets of several relatively novel anti-inflammatory drugs. Aside from these two major cytokines, Interleukin- 6 is also the subject of intensive investigation. Again it is primarily secreted by macrophages and is involved in the initiation of fever by crossing the blood brain barrier and stimulating the hypothalamus. Interleukin-17 is a relatively novel cytokine ${ }^{34}$. Unlike many other cytokines, it is produced by Thelper lymphocytes, and it stimulates the production of numerous other cytokines and chemokines by other leukocytes. Uniquely, it is often secreted during autoimmune diseases, leading investigators to propose IL-17 as a therapeutic target. The list of cytokines implicated in inflammation is large and expanding; intense interest in their role as therapeutic targets only reinforces their critical importance in inflammation.

Chemokines are a specialized subgroup of cytokines. They are small proteins, eight to ten kilodalton in size, that behave primarily as chemoattractants for different types of leukocytes ${ }^{7}$. To date, more than forty chemokines, overlapping over twenty corresponding receptors, have been described. They are classified according to the arrangement of their one or two pairs of crosslinked cystine residues. The $\mathrm{C}-\mathrm{X}-\mathrm{C}$ chemokines are primarily neutrophil chemoattractants; CXCL8 (formerly known as interleukin-8) is a prototypical member ${ }^{35}$. It is secreted by activated endothelial cells and macrophages and binds the CXCR1 and CXCR2 G protein-coupled receptors on neutrophils to stimulate migration and phagocytosis. The $\mathrm{C}-\mathrm{C}$ chemokines are more diverse are attract a range of leukocytes except neutrophils. Monocyte chemoattractant protein 1 (aka CCL2) is an essential recruiter of blood monocytes to sites of inflammation ${ }^{36}$. Eotaxin (aka CCL11) is unique in its ability to attract eosinophils, a rare blood-borne granulocyte important in airway inflammation and parasitic infections ${ }^{37}$. Other important family members include RANTES 
(regulated and normal T-cell expressed and secreted, CCL5) and macrophage inflammatory protein-1 $\alpha(C C L 3)^{38}$. The $C$ chemokines contain only one pair of cysteine crosslinks. Only two family members have been discovered and both primarily attract lymphocytes. The CX3C chemokine family contains only one member, fractalkine $(C X 3 C L 1)^{39}$. It is an unusually large protein at 42 kilodaltons and exists as both cell surface-bound or soluble forms. CX3CL1 is produced by endothelial and smooth muscle cells during inflammation to stimulate migration, adhesion and proliferation of monocytes, natural killer cells and T-lymphocytes. Overall, chemokines can be secreted constitutively in tissues under homeostatic conditions simply to organize different cells to different organ systems; however they are also strongly induced during inflammation and participate in eliciting leukocytes to sites of injury.

A majority of secreted inflammatory mediators is cell-derived, but the complement system and the coagulation system are two very specific systems of plasma-derived mediators that play an integral role in the escalation of inflammation ${ }^{14}$. Both systems are comprised of proteins expressed primarily in the liver and then circulated through the blood. The complement system consists of approximately twenty proteins numbered C1 through C9. While its primary functions are in the innate and adaptive immune response to microbial pathogens, its collateral effects during inflammation are profound. Three separate pathways exist: the classic pathway is triggered by binding of $\mathrm{C} 1$ to antibody-antigen complexes; the alternative pathway is activated by microbial surface antigens; the lectin pathway is stimulated by microbial surface carbohydrates ${ }^{11}$. All three pathways converge on the formation of enzyme C3 convertase which cleaves specific complement system proteins to form C3a, C3b and C5a. C3a exerts a major effect on inflammation through the direct stimulation of histamine release from mast cells. C3b can affix to microbial cell walls and act as opsonins to attract phagocytosing neutrophils and macrophages. C5a can activate lipoxygenases to synthesize powerful leukocyte-directing lipid mediators. Thus 
the complement system can produce certain proteins that contribute to the orchestration of inflammation. Separately, the coagulation system is also closely intertwined with inflammation ${ }^{40}$. The vasculature is normally a closed system, but physical rupture of epithelial surfaces such as the skin is both the primary opportunity for pathogenic invasion and most common active site for coagulation. The first phase of coagulation is platelet activation to form a platelet plug to stem hemorrhaging. Platelets degranulate serotonin, platelet activating factor and CXCL4, and generate $\mathrm{ADP}$ and $\mathrm{TxB}_{2}$, all secreted mediators described previously. Additionally, active thrombin, generated during the extrinsic clotting pathway, can enhance inflammation through proteaseactivated receptors (PARs) expressed on platelets, endothelial and smooth muscle cells to increase leukocyte adhesion receptor expression, cytokine and chemokine production, and NO and PAF generation. Additionally, the fragment fibrin byproducts of plasmin-catalyzed fibrinolysis can also enhance inflammation through interactions with $C 3$ in the complement system. In summary, the complement and coagulation systems are major plasma-derived biological processes with many common mediators of inflammation.

\section{Resolution of inflammation}

Once the original offending agents are contained, inflammation must resolve in order to allow tissue regeneration and restoration of homeostatic normality ${ }^{41}$. Historically, many well characterized mediators of inflammation were found to appear transiently; NO has a half-life of mere seconds, TxA2 is rapidly degraded as well, even the neutrophil is only present at inflammatory sites for one to two days before undergoing apoptosis ${ }^{7}$. Therefore it appeared selfevident that inflammation was a self-limiting process wherein mediators passively subsided once the source of injury was neutralized. However, the discovery of inflammatory mediators that only appear in the later phases of inflammation, such as IL-10 and lipoxin A4 (LxA4) has prompted the 
field to realign definitions and update terminology ${ }^{42}$. Two major classes of inflammation suppressing drug classes are the cyclooxygenase-inhibiting nonsteroidal anti-inflammatory drugs (NSAIDs) and the immunosuppressing corticosteroids ${ }^{40}$. By inhibiting cyclooxygenase, NSAIDs reduce the production of all eicosanoids, and by disrupting leukocyte maturation, proliferation and activation, corticosteroids diminish the synthesis of cytokines and chemokines. Since both classes of drugs actively inhibit the process of inflammation, a negative event, they are appropriately termed anti-inflammatory. However, the production of IL-10 and LXA4 are positive events $^{43}$. Therefore while they also oppose the propagation of inflammation, they are not just anti-inflammatory, but also pro-resolving, or promote resolution of inflammation. Within this new framework, the phagocytosis of microbes and necrotic and apoptotic cells, the egress of macrophages and dendritic cells from inflammatory sites to lymph nodes and the spleen, and the production of late-phase secreted mediators, are all pro-resolving actions occurring during the resolution phase of inflammation. Excessive production of inflammatory mediators, like reactive oxygen species, fever-inducing TNF $\alpha$, and excessive hemostatic plugs can exacerbate the original tissue injury, negating any positive effect of inflammation ${ }^{43}$. Thus resolution of inflammation must be timely implemented to tilt the inflammatory poise toward eventual restoration of tissue homeostasis.

A primary event in resolution is the secondary engulfment of apoptotic neutrophils that have already phagocytosed wound contents by monocyte-derived cells $s^{44}$. Due to their relative abundance circulating in blood, and their targeting by chemokines and adhesion molecules, neutrophils are the initial infiltrating cell type to inflammatory sites and thus the first to begin phagocytosing. Monocytes must be recruited from the splenic reservoir or differentiated from the bone marrow, thus they arrive approximately twenty-four hours later ${ }^{45}$. Although they also possess phagocytic capacity, monocytes can proliferate and differentiate into macrophages and 
dendritic cells that not only phagocytose but then egress to physically remove inflammatory debris. This collective function is termed efferocytosis ${ }^{46}$. What differentiates this secondary phagocytic activity from primary neutrophil phagocytosis of invading pathogens or dead cells is the lack of concurrent pro-inflammatory mediator release. When monocyte-derived cells ingest apoptotic neutrophil, they do not secrete cytokines or chemokines that recruit more leukocytes, such as TNF $\alpha$ or IL-8. They are instead non-phlogistic; they do not stimulate inflammation further ${ }^{47}$. As a result, monocyte-derived macrophages and dendritic cells, which appear later than neutrophils, are the ultimate cleansers of the damage site, blanching it of any cells or mediators that can elicit further inflammation. After phagocytic cleanup, they can migrate via blood or lymphatics to lymph nodes or the spleen for antigen presentation or complete degradation.

Corresponding to inflammation, resolution is also mediated by secreted mediators. Of these, IL-10 is the most prominent example of a resolution phase cytokine ${ }^{48}$. It is expressed primarily by monocytes and macrophages, and it elicits a host of effects that generally quell the propagation of inflammation. It interferes with the pluripotent inflammatory transcription factor nuclear factor kappa B (NF-kB) transcription pathway in leukocytes, thus severely diminishing the production of inflammatory mediators such as IFN- $\gamma$, TNF $\alpha$, IL-2, and GM-CS. IL-10 can also modulate the expression of cyclooxygenase 2, and thereby reduce synthesis of inflammatory prostaglandins and leukotrienes. Another important pro-resolving mediator is transforming growth factor $\beta(\text { TGF } \beta)^{49}$. Macrophage secreted TGF $\beta$ can inhibit inflammatory signaling of Tolllike receptors, which are important pattern recognition sensors of microbes. TGF $\beta$ can reduce the production of ROS by neutrophils. TGF $\beta$ can inhibit proliferation of IL-2 dependent T-cells and activation of cytotoxic T-cells. Finally, TGF $\beta$ can stimulate extracellular matrix deposition by mesenchymal cells; this is critical for repairing the pathogen, trauma and inflammation-damaged interstitium. However, the cellular actions of TGF $\beta$ are pleiotropic and not entirely pro-resolving, 
but the reason for this heterogeneity is still not well understood. Another pro-resolving mediator is the relatively novel Annexin $\mathrm{A} 1^{50}$. It can promote neutrophil apoptosis during inflammation and also stimulate their subsequent phagocytosis by macrophages. Neutrophils generate many secreted inflammatory mediators, such as ROS and hypochlorite, which have high collateral damage potential. Thus encouraging their quiet resignation also promotes the resolution of inflammation.

Lipid mediators are also produced during the resolution of inflammation. Just as TxB2 and PGI2 have counteracting vasoconstrictive and vasodilatory actions, several pro-resolving lipid mediators can oppose the inflammatory effects of prostaglandins and leukotrienes. Lipoxin A4 (LXA4) is a relatively novel but well characterized example of a pro-resolving lipid mediator ${ }^{29}$. It is biosynthesized from arachidonic acid in three main pathways: by 5 -lipoxygenase and 12lipoxygenase in leukocytes; by 15-lipoxygenase in parenchymal cells first then transcellularly though leukocyte 5-lipoxygenase; or from direct transformation by 5-lipoxygenase of membrane inositol-containing lipids stores of 5-HETE. The major actions of lipoxins are to decrease vascular permeability, decrease neutrophil-endothelium interactions and reduce neutrophil infiltration. LXA4 can also stimulate macrophage phagocytosis of microbes and apoptotic neutrophils. Overall, the major actions of LXA4 oppose the preceding actions of LTB4 and cysteinyl leukotrienes. Furthermore, since LXA4 sometimes share the same intermediate LTA4 substrate as LTB4, the diversion of AA products into LXA4 actually directly reduces LTB4 biosynthesis ${ }^{51}$. Another example of a resolution-phase lipid mediator is the resolvins ${ }^{52}$. These are also lipoxygenase products, but they are bioynthesized from eicosapentaenoic acid (EPA) and docosahexaenoic acid (DHA) precursors rather than arachidonic acid. While they have many actions similar to LXA4, they do not always bind the same $G$ protein-coupled receptors, and new actions are being actively researched. Resolvin E1 (RvE1) was the first EPA-series resolvin to be 
described $^{53}$. It is an EPA-lipoxygenase product that directly inhibits LTB4-stimulated neutrophil transmigration in vitro. RvE1 can inhibit both ADP-stimulated activation of P2Y12 and the effects of $\mathrm{TXB} 2$, to prevent platelet aggregation. In addition, RvE1 can directly block the transient receptor potential (TRP) family of nociceptors on skin keratinocytes and peripheral sensory neurons to mute inflammatory pain ${ }^{54}$. The $\mathrm{D}$-series resolvins are derived from DHA and share many effects with the E-series resolvins. One novel effect of resolvin D2 (RvD2), is the increase of antibiotic efficacy during infection without direct antimicrobial actions ${ }^{55}$. However, much remains left to be elucidated in pro-resolving lipid mediator biology, but interest in their unique resolution phase actions is intensifying and their importance in ushering a timely conclusion to inflammation is firmly established.

\section{Proliferation and remodeling after resolution}

The inflammatory response concludes with the proliferation and remodeling phase. Two broad processes occur during this phase that must be clearly separated: regeneration and repair ${ }^{7}$. Regeneration describes the proliferation of de novo cells and tissues that replace damaged structures and restore original functionality. Some amphibians are able to regenerate entire amputated limbs, but in mammals, regeneration is usually limited to small sections of liver or the bone marrow. More often, the process of repair concludes inflammation. What differentiates repair from regeneration is the formation of scar tissue rather than restoration of original function. Often the extent of tissue damage to the ECM and parenchymal cells, either from the original insult or from the resulting inflammation, is too extensive for the proliferation and remodeling phase to regenerate the affected tissue completely. Usually near the conclusion of the resolution phase of inflammation, the original pathogenic invaders and deceased host cells have all been confiscated from the damage site, and a repopulation by parenchymal and mesenchymal 
cells can proceed. The main mediators of this phase are macrophages, fibroblasts, vascular cells, progenitor cells, and the milieu of secreted mediators they produce ${ }^{7}$. Macrophages are pivotal in the initiation of the regenerative phase. While they phagocytose microbial and cellular debris, they are also secrete chemokines and growth factors to promote angiogenesis and extra cellular matrix remodeling ${ }^{56}$. In particular, the release of TGF $\beta$, platelet derived growth factor (PDGF), and fibroblast growth factor (FGF) signals the migration and then proliferation of fibroblasts at the wound site. Fibroblasts are a major type of mesenchymal cell with a branched plasma membrane and numerous rough endoplasmic reticulum ${ }^{57}$. They synthesize collagens, glycosaminoglycans, reticular and elastic fibers, glycoproteins found in the extracellular matrix. Initially, between 24 to $72 \mathrm{~h}$ of the regenerative phase, a provisional matrix of coagulation system-derived fibrin, plasma fibronectin and type III collagen is established by proliferating fibroblasts and endothelial cells. This temporary matrix of proteins, granulation tissue, contains leaky new blood vessels that allow abundant passage of plasma growth factors into the extravascular space, and gradually reconstitutes the debrided tissue damage site. Slowly and under stimulation primarily by TGF $\beta$, this granulation tissue is replaced with scar tissue through connective tissue remodeling by matrix metalloproteinases. Fibroblasts and macrophages produce these digestive enzymes, which degrade the damaged and provisional ECM while the new collagen I matrix is deposited by fibroblasts.

In addition to ECM repopulation, the original parenchymal cells of the damaged tissue also begin to reappear through the proliferation and differentiation of various progenitor cells ${ }^{58}$. These cells can be hematopoetic stem cells, mesenchymal stem cells, or a range of tissue-specific progenitor cells. This is a relatively novel field in biology and the full lineage characterization of the various cell types is incomplete. Some features of these cells have become well accepted however. It is generally believed that they are generally small cells with large round nuclei and few 
cytoplasmic organelles; they have a much greater capacity for self-renewal than somatic cells; some of their regenerative effects are not autologous but through paracrine effects on existing tissue cells; and they are highly plastic, with the ability to differentiate into a variety of parenchymal cell types or de-differentiate into a more pluripotent embryonic state. Often the regeneration of damage tissue is incomplete as accumulation of new collagen displaces leukocytes and leaky vasculature, and converts the area into a pale, avascular scar that does not retain the original tissue function. This is frequently the outcome of deep cutaneous wounds ${ }^{7}$. However, other tissues such as the liver are able to regenerate with restored function. Of course, there are many anatomical and cellular differences between the skin and the liver, but this disparity in regenerative capability remains one of the most compelling questions in biology. New and incoming insights from stem cell research have the potential to address this question.

\section{Wound Healing}

Wound healing is a dynamic process involving multi-modal interactions of resident and infiltrating cells, secreted mediators and extracellular matrix molecules tasked with the immediate goal of maintaining tissue integrity and eventually returning to homeostasis ${ }^{59}$. As such, it shares many processes with the inflammatory response, including the recognition of tissue damage, infiltration of leukocytes to remove debris, and the establishment of granulation tissue. However wound healing also involves other reparative and regenerative processes. In cutaneous wounds, wound contraction and recovery of tensile strength are instrumental in healing. Contraction of a surface wound decreases the gap between dermal edges and thus reduces wound surface area. A network of myofibroblasts, bearing many similarities to smooth muscle cells, form at the wound edge and produce large quantities of type I collagen, tenascin- $C$, osteonectin and fibronectin that comprise extracellular matrix under the stimulation of macrophage secreted TGF $\beta$, PDGF, and 
$\mathrm{FGF}^{60}$. After the initial week of ECM deposition, the tensile strength of the contracted wound is merely $10 \%$ of undamaged skin $^{7}$. Months later, as collagen fibers are increased in size and crosslinked together; tensile strength is restored to about $70-80 \%$ of the original skin. The ultimate recovery of cutaneous wounds is dependent on systemic and local host factors. Nutrition derangements, either protein or vitamin deficiency, or nutrient overload in diabetes and obesity, can alter significantly the timecourse or magnitude of wound healing. Compromises in circulatory status, such as those caused by atherosclerosis or varicose veins, can also impair wound healing. Locally, pathogens present the largest obstacle to proper healing, but mechanical interference at the wound site, foreign material, and wound size and location, are factors that can interfere with the orderly and timely healing of wounds.

\section{Revascularization}

Another component of wound healing is the restoration blood perfusion through vasculogenesis, angiogenesis and arteriogenesis ${ }^{61}$. Vasculogenesis is the de novo formation of blood vessels from incompletely differentiated but endothelial-committed progenitor cells where no prior vasculature existed. Angiogenesis is the sprouting of new capillaries from existing capillary networks by branching or recruited progenitor cells. And arteriogenesis is the diameter expansion of existing arteriolar connections into true collateral arteries. Vasculogenesis occurs predominantly during the embryonic and fetal period ${ }^{62}$. During gastrulation, ectodermal cells are recruited to the primitive streak and undergo an epithelial to mesenchymal transition. These cells then migrate between the visceral endoderm and epiblast, and form mesoderm through bone morphogenic protein 4 (BMP4) and fibroblast growth factor 2 (FGF2) stimulation. The visceral endoderm then secretes the growth factors indian hedgehog (IHH) and FGF2 that target the mesoderm to form primitive endothelial and hematopoietic cells. These cells express VEFG 
receptor 2 (VEGFR2) and respond to visceral endoderm VEGF to coalesce and form blood islands that fuse to mature into a primitive network of capillaries or vascular plexi. Once these nascent vascular complexes form, they are remodeled by a delicate balance of cell-cell and cell-matrix signals and interactions to establish a mature circulatory network ${ }^{63}$. Important growth factors during this process include VEGF and FGF2, but also retinoic acid and TGF $\beta$, and fibronectin binding alpha $\mathrm{V}$ beta $1(\mathrm{aV} \beta 1)$ and alpha $\mathrm{V}$ beta 3 (aV $\beta 3$ ) integrins. Additionally, smooth muscle cells and pericytes are recruited to wrap the outer blood vessel walls and add contractile function. Eventually as the fetus grows, vasculogenesis is supplemented with the angiogenic sprouting of existing circulatory networks.

Once again, VEGF (and to a lesser degree FGF2) secreted by mesenchymal and stromal cells remains the primary driver of angiogenesis. VEGFR2 is again the principal receptor for VEGF ${ }^{64}$. Through this signaling pair, endothelial progenitor cells are recruited from the bone marrow and induced to proliferate, differentiate and chemotaxi into new capillary sprouts. The actual process of capillary networking is still under investigation; however, a group of Notch ligands (Jagged 1 and 2, Delta-like 1, 3 and 4) and receptors (Notch 1-4)have been found to be critical mediators $^{65}$. During the sprouting process, the leading angiogenic progenitor cell, referred to as a tip cell, proliferates and migrates under VEGF stimulation. They also produce delta-like ligand 4, which interacts with Notch 1 and 4 on the stalk cells that remain tethered to the existing capillary. Ligand triggering of stalk cell Notch receptors leads to receptor downregulation and nuclear translocation where genes that dampen VEGF responsiveness are activated. Thus through a signaling loop, tip cells sprout forward while leaving a tube of stalk cells that form a new vessel. Once formed, new vessels remain fragile until they are stabilized by the recruitment of pericytes and smooth muscle cells and the deposition of ECM proteins. Angiopoietins, PDGF, and TGF $\beta$ are the principal mediators of stabilization ${ }^{66}$. Angiopoietins recruit pericytes while PDGF recruits 
smooth muscle cells. TGF $\beta$ enhances the deposition of ECM molecules and expression of their cellular receptors. The formation of a robust ECM is critical in modulating angiogenesis and protecting the integrity of the new vessels. Many other mediators, and hypoxic stimulus, have been found to enhance angiogenesis. $\alpha \mathrm{V} \beta 3$ integrin is stimulated by hypoxia, potentiates metalloproteinase activity, and interacts with fibronectin and vitronectin to assist cell spreading and migration ${ }^{67}$. Metalloproteinases and plasminogen activators allow endothelial cells to remodel the ECM and invade new angiogenic space ${ }^{68}$. Cytokines and chemokines, such as IL-6, IL8, CCL2, and CXCL12, can induce angiogenesis as well ${ }^{69}$. The list of molecules that stimulate angiogenesis is constantly expanding. Some are only expressed during the fetal period; others are only induced in tumor environments. Some molecules directly enhance angiogenesis through cell-matrix interactions, others only stimulate indirectly through increases in VEGF ${ }^{70}$. Regardless of stimuli, angiogenesis is a complex cellular process that is integral to wound healing.

Internal tissue wounds are often the result of ischemia, a restriction in blood supply, rather than trauma or infection. In cases of large artery occlusion, the process of angiogenesis, which develops over the course of days, is insufficient to rescue ischemic tissue or organs ${ }^{71}$. Early clinical trials testing the efficacy of VEGF stimulated angiogenesis in coronary heart disease patients was largely unsuccessful ${ }^{72}$. Instead, it is collateral arterioles, rather than capillaries, that must expand in size to compensate for the decreased blood flow. This expansion is not just vasodilation. The development of collateral arterioles into collateral arteries involves proliferation of endothelial and smooth muscle cells, hence the name arteriogenesis ${ }^{73}$. Because the occlusion is not in the collateral arteriole itself, ischemia, and therefore hypoxia, is not the primary driving force of arteriogenesis. Instead there is an increase in blood fluid shear stress as the collateral arterioles now more directly connect high-pressure vessels with low-pressure capillaries. This stress stimulates endothelial cell to produce CCL2 to recruit proliferative mediator-producing 
monocytes ${ }^{74}$. The resulting expanded collaterals possess numerous dividing endothelial and smooth muscle cells, forming a cell-rich intima. They also adopt a highly characteristic tortuosity as the vessel grows in both width and length. Unfortunately, even the normal arteriogenic process occurs over the course of days, which is insufficient to rescue such severe cases of ischemia as myocardial infarction or stroke where irreversible damage occurs within minutes to hours. Moreover, arteriogenesis is a self-limiting process ${ }^{75}$. When the collateral arteriole expands, the amount of fluid sheer stress stimulus decreases and tapers the vessel's further expansion. Under normal physiological conditions, arteriogenic restoration of perfusion is therefore asymptotic, but given external stimuli, such as more fluid shear stress or other soluble mediator, arteriogenic enlargement can be enhanced. Thus, while surgical and endovascular revascularization has been brilliantly effective in redressing ischemia and rescuing lives, the enhancement of biological revascularization, arteriogenesis and angiogenesis, can further improve wound healing outcomes.

\section{Monocytes and Macrophages}

Monocytes and macrophages play an important role in the revascularization process by regulate both inflammation and wound healing. These cells appear early during embryogenesis ${ }^{76}$. Primitive macrophages are in the yolk sac and were first identified by Ilya Mechnikov who described the process of phagocytosis by a large (macro) eater (phage) cell in starfish larvae ${ }^{77}$. Subsequent work has led to the identification of the origin and fate of these primitive macrophages $^{76}$. In particular, it has been shown that as development progresses and hematopoiesis begins in the fetal liver, hematopoietic stem cells produce a common myeloid progenitor cell, which in turn form monoblasts that eventually mature into monocytes. These monocytes are then distributed throughout the fetus in several tissues and differentiate into tissue-specific macrophages. Historically, macrophages have been defined histologically, and 
therefore have inconsistent names: histiocyte, Kupffer cell, Langerhans cell, microglia, hofbauer cell, mesangial cell, osteoclast, epitheloid cell. However, subsequent molecular and genetic analysis has confirmed their myeloid-derived lineage. Once the fetus matures into a newborn (and then adult), hematopoesis shifts from the liver and spleen to the bone marrow. Subsequent production of monoblasts, and therefore monocytes and macrophages, then occur from myeloid progenitor cells in the bone marrow. Also, although it was once believed that tissue-resident macrophages were entirely derived from hematopoetic cells and that their tissue reservoirs is replenished when hematopoiesis shifts to the bone marrow, recent evidence suggests that reservoirs of tissue-specific macrophages can be maintained through local self-proliferation ${ }^{78}$.

There is recent consensus that both monocytes and macrophages could be broadly categorized into two different states: an activated and inflammatory state; and a resident and reparative state ${ }^{79,80}$. The two states of monocytes are defined by surface markers; that could be easy detection by flow cytometry of peripheral blood. Inflammatory monocytes are larger and express CCR2 (denoted CCR2 ${ }^{+}$) chemokine receptor, L-selectin, and high levels of Ly-6C (denoted Ly- $\left.6 C^{\text {hi }}\right)$. Resident monocytes are smaller and express much lower levels of Ly- $6 C\left(L y-6 C^{l o}\right)$, no CCR2 (CCR2) or L-selectin, but instead express the fractalkine receptor CX3CR1. Inflammatory monocytes normally represent $2-5 \%$ of circulating leukocytes, and they home rapidly to sites of inflammation largely because of their CCR2 receptors. Resident monocytes are less prevalent in the peripheral blood but they have a unique ability to migrate along the luminal surface of endothelial cells and patrol the vasculature. Both types of cells are derived from bone marrow monocytes but a splenic reservoir of both types of differentiated monocytes exists for rapid deployment when needed ${ }^{45}$. Just like their blood-borne originant, macrophages can exist in two polarized states.

The classically activated macrophage respond to microbial antigens, have strong 
microbicidal activity, produce inflammatory cytokines, release matrix metalloproteinases, and respond to T-helper type I cytokines, primarily IFN- $\gamma^{80}$. Two decades ago, Gordon et al, discovered that IL-4 induces macrophages to adopt a unique phenotype associated with the production of growth factors (PDGF and TGF $\beta$ ), inhibition of metalloproteinase activity, and responsiveness to Thelper type II cytokines, such as IL-4 and also IL-13 ${ }^{81}$. These two subsets of macrophages were categorized as M1 or classically activated, and M2 or alternatively activated ${ }^{82}$. Specific markers of these two subsets have been described; two markers that represent the two subsets particularly well are inducible nitric oxide synthase (iNOS) and arginase ${ }^{83}$. Both these enzymes compete for arginine: iNOS converts arginine into nitric oxide, while arginase converts arginine into ornithine and urea. Conversion of arginine to NO is required for both inflammatory vasodilation and killing of microbes - two important key events that characterize the onset of inflammation. The arginase product ornithine is a precursor of proline, the second most common amino acid in collagen. As collagen is the primary constituent of extracellular matrices, ensuring its consistent supply is critically important during the proliferative and remodeling phase of inflammation. Therefore, the two subsets of macrophages have been classified as M1 macrophages, which are inflammatory and destructive while the M2 macrophages are pro-resolving and reparative.

The existence of two subsets of monocytes and macrophages has led to assumption that inflammatory monocytes $\left(\mathrm{Ly}-6 \mathrm{C}^{\mathrm{hi}}, \mathrm{CCR} 2^{+}\right)$differentiate into $\mathrm{M} 1$ macrophages and resident monocytes $\left(\mathrm{Ly}-6 \mathrm{C}^{\mathrm{lo}}, \mathrm{CCR} 2^{-}, \mathrm{CX} 3 \mathrm{CR} 1^{+}\right)$differentiate into M2 macrophages ${ }^{84}$. Nevertheless, this segregation is intensely debated and recent evidence suggests otherwise. Detailed tracking studies involving the monocytes and macrophages in blood and in tissue provide strong evidence that during the onset of inflammation inflammatory monocytes are recruited to afflicted tissue where they participate in pathogen neutralization before transforming into resting monocytes that then mediate the resolution of inflammation ${ }^{78}$. Eventually these pro-resolving resting 
monocytes differentiate fully into reparative macrophages during the proliferative and remodeling phase of inflammation. These reparative macrophages display a combination of markers in between the polarized M1 and M2 definitions however. While this is somewhat conflicting, it is important to qualify that M1 and M2 phenotypes are largely defined by their in vitro characteristics. Stimulation with recombinant IFNy or IL-4 can induce M1 and M2 polarization in cultured macrophages, and specific isolated in vivo populations of macrophages can variously display $\mathrm{M} 1$ or $\mathrm{M} 2$ markers $^{80}$. However, macrophages are highly plastic cells that are capable of shifting between the extreme categories of M1 and M2 in a heterogenous continuum in vivo, and it is being increasing recognized that macrophages assume a wide spectrum of phenotypic characteristics with M1 and M2 representing the two most polarized states ${ }^{84}$.

Several studies have shown that transplantation of exogenous monocytes and macrophages promote tissue healing and repair in models of tissue infection and injury ${ }^{85}$. Because these studies use different types of monocytes or macrophages, the extent of protection observed is variable. Thus improving the definition of monocyte and macrophage subsets could help in developing more targeted interventions. An area of particularly urgent clinical need is therapeutic revascularization. Monocytes and macrophages are necessary for revascularization, and their supplementation can enhance revascularization. Loss-of-function studies in which monocytes or macrophages were depleted by pharmaceutical intervention or genetic manipulation before ischemia have shown significant defects in revascularization. In comparable gain-of-function studies wherein monocytes or macrophages were supplemented before and or after ischemia, usually by intravenous injection, marked improvements in revascularization were demonstrated. The consensus is that monocytes and macrophages improve revascularization via three main mechanisms ${ }^{86}$ : (1) generation of arteriogenic and angiogenic growth factors and soluble mediators; (2) remodelling of the extracellular matrix of vasculature; and (3) consolidating 
into the structural constituents of the vascular wall. Given the highly heterogenous and plastic nature of these cells, these three mechanisms are hardly surprising in the diversity and reach. Finally, the evidence supporting that the notion that monocyte and macrophage transplantation enhances revascularization is not entirely derived from laboratory animals, as transplantation of monocytes or macrophages have also demonstrated improvements in revascularization in human clinical trials as well ${ }^{85}$. Nevertheless, the two important questions remain unanswered: What subsets of monocyte or macrophage enhance revascularization? And what are their most effective secreted mediators that promote revascularization?

The first question has already been extensively addressed, though the answer is ambiguous and slightly anti-climactic. Many subsets of monocyte or macrophage have the ability to improve revascularization. However, since there is variability in disease model, method of cell collection, and time of supplementation, the efficacy of these subsets cannot be directly compared ${ }^{85}$. Monocytes and macrophages exist in many different body depots: blood, spleen, peritoneum, and bone marrow. Given their highly plastic nature, the cells that populate these depots are heterogenous and exist on a shifting continuum of subsets. The method of collection, purification and amplification of these cell depots generates further variability. Furthermore, the time point(s) at which this heterogenous mix of cells is supplemented, in relation to the original occlusive injury, can have significant impact on outcome. One biomedical company, Aastrom Biosciences, is currently testing its proprietary monocyte and macrophage mixture, ixmyelocel-T, for treatment of critical limb ischemia (CLI) in phase 3 clinical trials ${ }^{87}$. Preliminary data from earlier trials suggest there could be some reduction in adverse CLI outcomes, indicating that revascularization-enhancing monocyte and macrophage cell therapy has promising clinical potential. The final verdict must wait until the estimated study completion date in March 2015. Meanwhile, there are several major caveats to cell-based therapy: it is expensive to administer, 
requires autologous donor cells, subject to isolated cell variability, and poses immunogenicity or contamination risks ${ }^{85}$. Whenever possible, a pharmaceutical alternative is more effective and less fickle. Therefore the second question of most effective pharmaceutically adaptable revascularization-enhancing mediator is very pressing.

VEGF and FGF are critical mediators of angiogenesis and they are small proteins, recommending them as obvious revascularization-enhancing candidates for pharmaceutical adaptation $^{72}$. Various forms of VEGF and FGF were developed: recombinant protein, naked DNA, gene-containing plasmid. And various methods of administration were tested: intravenous, intramuscular, and intracoronary. Thus far, no combination of approaches has yielded an FDAapproved drug. Many other secreted mediators have been the subject of candidate drug trials, but none has yet to be approved.

One family of secreted mediators vying for pharmaceutical candidacy in the enhancement of post-ischemia revascularization is the pro-resolving lipid mediators. Traditional prostaglandins are already employed in the clinic for various vascular anomalies: PGE1 as alprostadil; PGE2 as dinoprostone; PGF2 $\alpha$ as dinoprost; and PGI2 as epoprostenol ${ }^{40}$. Thus pro-resolving lipid mediators have reliable precedents set for its translation. Furthermore, recent studies have directly tested its potential for revascularization enhancement. In a rat model of myocardial ischemia, resolvin E1 (RvE1) administration thirty minutes after ischemia attenuated cardiac infarct size ${ }^{88}$. In a mouse model of renal ischemia-reperfusion injury, RvD1 treated mice also experienced attenuated drops in kidney function ${ }^{89}$. What remains unclear though, are the specific mechanisms by which resolvins enhance revascularization. Another two key questions remain: what are the cells that produce these revascularization-enhancing pro-resolving lipid mediators? and what are the cellular processes that these biosynthesized lipid mediators then stimulate? 


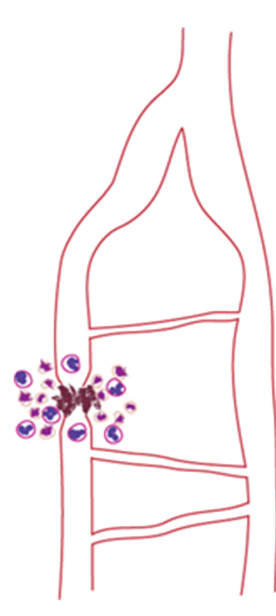

mast cells

neutrophils

Ly-6Chi CCR2+ monocytes

histamine TNF $\alpha$

ROS IL- $1 \beta$

NO PGE2

LTB4

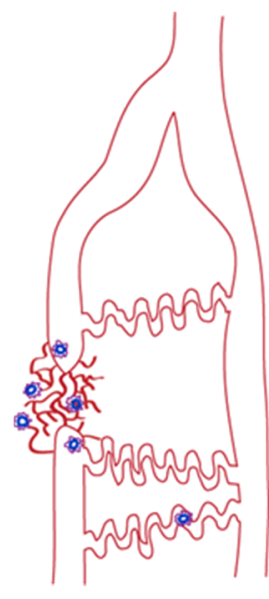

IL-10

TGF $\beta$ LXA4 RvD2

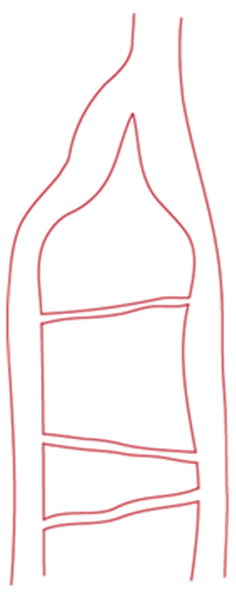

macrophages

Ly-6Clo CCR2- monocytes

TGF $\beta$

VEGF

bFGF

PDGF 


\section{Projective objective}

Impaired tissue perfusion is a pathologic feature of many prevalent chronic diseases, including peripheral artery disease and diabetes, which can precipitate tissue necrosis or limb amputation $^{90}$. Several clinical strategies have been designed to increase the revascularization of ischemic tissue in these diseases. Treatments include endovascular angioplasty, bypass grafting, and delivery of specific angiogenic agents (VEGF, bFGF, GM-CSF). Recently, cell therapy involving the autologous supplementation of exogenously amplified mononuclear or progenitor cells, have been used to promote revascularization ${ }^{87}$. Although often successful, these therapies have limited efficacy in part because they do not address the underlying chronic inflammation, which is often the primary feature of diseases involving impaired tissue perfusion. Furthermore, angiogenic agents are often also mitogenic and therefore possess the ability to exacerbate the underlying chronic inflammation ${ }^{91}$. Therefore new therapeutic candidates are needed to promote revascularization of ischemic tissue and concomitantly stimulate the resolution of chronic inflammation.

The resolution of inflammation is an essential process within an animal's self-regenerative ability to address injuries incurred during their lives. While the form of injury, pathogenic infection, traumatic wounding, ischemic damage, varies, a broadly common program of biological processes occurs in response. Inflammation is the initiating process wherein leukocytes infiltrate from the blood to contain the injury and remove damaged tissue. Resolution follows inflammation; structural and vascular cells migrate to create a provisional tissue scaffold and restore blood supply. The final process of wound healing is regeneration, where the initial structural components are consolidated and repopulated. Since wound healing begins with inflammation, the timely conclusion of this phase is essential. The prevailing dogma holds that inflammation resolves passively following dissipation of pro-inflammatory stimuli. However, 
emerging evidence suggests that the resolution of inflammation is an active process, orchestrated by in part by lipid mediators and involving a "pro-resolving program" that counteracts ongoing inflammation ${ }^{43}$. Due to the leading role of inflammation in the wound healing process, proresolving lipid mediators are thus hypothesized to be important proponents of the return to homeostasis. These pro-resolving lipid mediators are generated during inflammation by leukocytes and have direct actions on monocytes/macrophages (stimulating phagocytosis) and endothelial cells (nitric oxide production) ${ }^{55}$. Other investigators report that resolvins are generated during kidney model of ischemic injury and 12/15 lipoxygenase KO mice (an enzyme involved in resolvin synthesis) experience impaired recovery from hind limb ischemia injury ${ }^{92}$. Therefore the resolvin pathway is directly implicated in the neovascularization process of wound healing.

In this series of investigations, I hypothesize that during the wound healing process, leukocyte-derived pro-resolving lipid mediators promote tissue revascularization by stimulating arteriogenesis and angiogenesis. To test this hypothesis, we will assess whether exogenous delivery of pro-resolving lipid mediator resolvin D2 (RvD2) will enhance revascularization using a murine model of hind limb ischemia. In ischemic limbs, we will assess the recovery of limb blood flow by laser Doppler perfusion imaging and measure increases in arteriogenesis and angiogenesis by Microfil injection and micro-computed tomography of hind limbs. To examine the cellular biosynthesis of RvD2 during revascularization, we will determine whether RvD2 is generated during revascularization, and whether monocytes and macrophages produce RvD2. Finally, to provide evidence of RvD2-stimulated arteriogenesis and angiogenesis, we will examine RvD2-induced changes in gene transcription in monocytes, macrophages and endothelial cells. We will use the Matrigel plug assay to assess the direct angiogenicity of RvD2 in vivo. To identify the endothelial effects of RvD2, we will measure the stimulation of endothelial migration and proliferation by RvD2. Collectively, these studies will establish whether generation of pro- 
resolving lipid mediators play a role in wound revascularization. Results of these studies could form the basis for the development of novel therapeutics aimed at improving revascularization in major clinical occlusive crises such as myocardial infarction, stroke and critical limb ischemia, and in conditions associated with altered wound healing, such as peripheral vascular disease and diabetes. 


\section{CHAPTER II}

\section{RESOLVIN D2 ENHANCES POST-OCCLUSION REVASCULARIZATION}

\section{Introduction}

Inflammation is a dynamic process mediated by quorums of danger sensors, secreted mediators, and resident and infiltrating cells. It can combat a remarkably diverse armada of pathogenic invaders, and it can redress a wide spectrum of traumatic or ischemic injuries. Unfortunately, inflammation can also produce collateral damage and it may not always promote a full restoration of function. The improper sensing of benign antigens, followed by an exaggerated inflammatory response, underlies the mortality of anaphylactic reactions ${ }^{7}$. Deep cutaneous wounds do not heal completely; they are covered by a reduced thickness fibrotic scar ${ }^{7}$. Often inflammation does not resolve promptly, perpetuating a chronically active state ${ }^{7}$. Prominent clinical examples of this unremitting state include autoimmune diseases, atherosclerosis, diabetes, Alzheimer's disease, lung fibrosis, and cancer. In essence inflammation prevents infections and promotes healing, but if the process becomes deranged it can cause injury and disease itself.

The intuitive approach to combat unresolved inflammation clinically would be to inhibit further escalation of inflammation. For decades, medical treatments focused on inhibiting inflammation via agents that antagonize enzymes, receptors, genes or cytokines responsible for its propagation. For example, anti-histamines block histamine receptors to quell allergic reactions $^{93}$. Non-steroidal anti-inflammatory drugs block COX-1 and COX-2-dependent production of prostaglandins ${ }^{94}$. Synthetic glucocorticoids interfere with pro-inflammatory gene expression and directly induce apoptosis in lymphocytes ${ }^{95}$. Infliximab and canakinumab are 
monoclonal antibody therapies that neutralize pro-inflammatory TNF- $\alpha$ and IL-1 $\beta$, respectively ${ }^{96}$. Thus, agents that block the inflammation are some of the most commonly used drugs in clinical medicine. Nevertheless, even though these drugs can be highly effective in acute situations, blocking inflammation chronically carries significant adverse effects. Prolonged NSAID use can cause gastric ulcers and liver damage ${ }^{97}$. Selective COX-2 inhibitors can actually increase cardiovascular mortality ${ }^{98}$. Glucocorticoids cause lipodystrophy and induce a dangerously immunocompromised state ${ }^{99}$. As a result, new and alternative strategies for modulating the inflammatory response are being sought actively. One novel approach is to promote resolution of inflammation (i.e., agonism) rather than simply shutting it down (i.e, antagonism).

Polyunsaturated fatty acids have been extensively studied for potential resolutionpromoting actions and the recent discovery of pro-resolution lipid mediators derived from $\omega-3$ PUFAs has established a new class of compounds with therapeutic potential. Decades of human trials have already made a strong case for further promotion of cardiovascular health by $\omega-3$ PUFAs. In 1956, Sinclair postulated that low rates of atherosclerosis in the Canadian Inuit were a result of their high consumption of marine fat ${ }^{100}$. A much more systematic series of studies were completed by Bang and Dyerberg in 1970 s across the bay in Greenland Inuits ${ }^{101,102}$. They found that while the total fat percentage of Greenland Inuit diets were nearly identical to Danish diets, the Inuit consumed a much higher percentage of $\omega-3$ fatty acids while the Danish consumed more saturated fats. The higher consumption of $\omega-3$ fatty acids (DHA and EPA) corresponded to higher composition of $\omega-3$ fatty acids in Inuit blood platelets. Remarkably, these Inuit also had significantly lower plasma triglycerides and higher high-density lipoprotein. In a morbidity survey spanning 25 years in 1800 Inuit, the incidence of myocardial infarction was ten fold lower than the Danish population. Across the world, Japanese Okinawans with similar $\omega-3$ diets also had low rates of cardiovascular disease ${ }^{103}$. Prompted by these natural experiments, the Diet and 
Reinfarction Trial (DART) in 1989 found a decrease in both cardiovascular disease-related death and all-cause mortality in post-MI non-diabetic men consuming $\sim 300 \mathrm{~g}$ of fatty fish a week ${ }^{104}$. Unfortunately, large populations of humans live far from ocean coasts and do not consume a high $\omega-3$ fish diet. Fortunately, supplementation of $\omega-3$ fatty acids in pill form is much easier. Thus the Gruppo Italiano per lo Studio della Sopravvivenza nell'Infarto miocardico (GISSI) Prevenzione trial, enrolled over 11,000 patients with a history of myocardial infarction, and in the active treatment group, demonstrated reduced risk of cardiovascular death in those consuming $850-882 \mathrm{mg}$ of EPA and DHA per day ${ }^{105}$. More recently, the GISSI-heart failure trial further concluded that fish oil supplementation decreased mortality in heart failure patients ${ }^{106}$. Although the DART and GISSI trials are the two largest clinical trials involving $\omega-3$ fatty acids, many other smaller trials with different test criteria and endpoints have also been performed. A meta-analysis of their combined results found substantial variation in total mortality and no evidence of substantial benefit of $\omega-3$ fatty acids on combined cardiovascular mortality ${ }^{107}$. While this conclusion is discouraging, it is important to note the large numbers of confounding factors, such as study size, treatment methodology, and patient compliance can obfuscate results and cloud conclusions. Unlike the equivocal conclusions of the human trials, an unequivocal solution is to further research into the mechanisms underlying the beneficial cardiovascular effects of $\omega-3$ fatty acids.

The $\omega-3$ fatty acids EPA and DHA are bioactive and may directly contribute to cardiovascular death reduction, but they are also substrates for cyclooxygenases and lipoxygenases like the $\omega-6$ arachidonic acid (AA). Therefore the health benefits of $\omega-3$ fatty acids may not be related to direct actions, but are mediated through the actions of their metabolites. For example, EPA can be converted to 3-series prostaglandins and 5-series leukotrienes that are less biologically potent than their AA counterparts ${ }^{108}$. When they flux through the normal biosynthetic pathways, they reduce the production of pro-inflammatory AA-derived mediators 
though substrate competition. The physiological result is that in a high $\omega-3$ diet, with the same level of other inflammatory stimuli and mediators, less potent inflammatory responses are generated. As described in the previous chapter, this reduced response is not always detrimental. In fact during fetal cutaneous wound healing, the immature mast cell response allows the wound to heal without scarring ${ }^{109}$. The recently created fat- 1 transgenic mouse, which expressed a desaturase enzyme enabling endogenous $\omega-6$ to $\omega-3$ fatty acid conversion, is protected from inflammatory pathologies such as colitis and asthma ${ }^{110}$. So far, this evidence suggests that $\omega-3$ fatty acids possess mostly anti-inflammatory properties. However, the discovery of E-series and Dseries of resolvins ${ }^{111}$, coupled with existing knowledge of the anti-inflammatory but also proresolving actions of lipoxin $A 4$ (derived from $A A$ ), has led to the proposal that local biosynthesis of bioactive lipid mediators could underlie some of the health-benefitting immunomodulatory effects of $\omega-3$ fatty acids.

Resolvins have been extensively studied, and their basic characteristics have been widely reported, more immunomodulatory actions are being described. Recent work has identified the biosynthetic pathway (to be discussed in the next chapter) and the cellular receptors for some resolvins $^{112}$. Is has been shown that EPA-derived RvE1 binds to ChemR23, a GPCR with about 36\% sequence homology to the lipoxin A4 receptor (ALX). It also binds to BLT1, another GPCR that binds also LTB4. Interestingly, RvE1 acts as an inverse agonist of BLT1, preventing the inflammatory actions of LTB4. The DHA-derived RvD1 binds to ALX and GPR32 receptors in humans ${ }^{113}$. Stimulation of human macrophages with RvD1 enhanced their phagocytosis of apoptotic neutrophils and yeast zymosan. RNAi knockdown of either receptor decreased phagocytosis rates and overexpression of each further enhanced phagocytosis rates. Many of the biological actions of RvE1 are summarized in Chapter 1. RvD1 also possess potent bioactivity ${ }^{114}$. In microfluidic chamber experiments, RvD1 was found to halt neutrophil chemotaxis towards CXCL8 
and adherence to endothelial cells. During mouse peritonitis, RvD1 can modulate microRNAs, increasing miR-21, miR-146b and miR-219 expression while decreasing miR-208a. It has also been shown that RvD1, like RvE1, can block inflammatory pain, but antagonizes a different set of transient receptor potential channels: TRPV3, TRPV4 and TRPA1. In a mouse model of obesityinduced steatohepatitis, RvD1 administration was able to increase adiponectin expression, reduce macrophage infiltration, skew existing macrophages into a M2 phenotype, and reduce expression of COX2, IL1 $\beta$, IL- 6 and CCR7 mRNA ${ }^{115}$. In a complementary obesity study by our research group, RvD1 treatment of leptin receptor-deficient diabetic mice increased insulin sensitivity, adiponectin levels and insulin-stimulated Akt phosphorylation in adipose tissue. RvD1 also reduced crown-like structures, granuloma-resembling masses of apoptosis adipocytes phagocytosing M1 macrophages, and instead increased the presence of M2 macrophages in adipose tissue. In a follow-up study by our group, RvD1 treatment attenuated the chronic inflammatory diabetic state by reducing accumulation of apoptotic thymocytes, enhancing the resolution of acute peritonitis, and stimulating Fc receptor-mediated macrophage phagocytosis ${ }^{116}$. Additionally, we documented decreased production of resolvin-precursors in skin wounds of these diabetic mice. We found that when RvD1 was topically applied to wounds, wound closure accelerated and fewer apoptotic cells and macrophages accumulated. As defective wound healing is a serious concern in diabetics, a topical healing accelerator represents an attractive therapeutic strategy. Finally, given the pressing global obesity epidemic ${ }^{117}$, and the extant cardiovascular crisis, RvD1 might be a promising candidate for pharmaceutical therapy to resolve inflammation and promote resolution.

Resolvin D1 was the first D-series resolvins to be described. Since its discovery, other Dseries resolvins have been characterized. Resolvin D2 (RvD2) is a regioisomer of RvD1 that is also present during the resolution phase of inflammation ${ }^{55}$. In the mouse acute peritonitis model, RvD2 also reduced peritoneal neutrophil infiltration, and it stimulated macrophage phagocytosis 
of zymosan particles. In a mouse cecal-ligation sepsis model, RvD2-treated mice experienced lesser microbial spillover into the peripheral blood and peritoneum, and experienced greater overall survival rates. Peritoneal neutrophil numbers, as well as inflammatory mediators TNF- $\alpha$, IL1ß, IL-6, IL-23, IL-17, PGE2 and LTB4, were decreased as well. Additionally, as previously mentioned, while RvD2 has no direct microbicidal effect like NO, it nevertheless enhanced the ability of leukocytes to contain the leaked bacteria. Some of these outcomes resemble RvD1stimulated effects, others are unique. Especially novel and intriguing are its vascular actions. In the exposed mouse cremaster muscle microcirulatory model, RvD2 reduced platelet activating factorstimulated neutrophil adherence and extravasation. Further addition of the NO synthase inhibitor, L-NAME, restored the basal effects of PAF on neutrophils. In a separate in vitro experiment, RvD2 also stimulated NO generation by human umbilical vein endothelial cells. Collectively, RvD2 has a distinctive assortment of immunomodulatory and vascular actions. Coincidently, these actions intersect with the immunovascular process of arteriogenesis and angiogenesis after tissue injury. Because $\omega-3$ fatty acid supplementation can reduce adverse cardiovascular outcomes, and $\omega-3$ fatty acids can be metabolized into RvD2, it is likely that the cardiovascular benefits of $\omega-3$ fatty acid supplementation may be mediated by the immunovascular effects of RvD2.

Critical limb ischemia is an adverse cardiovascular outcome, and a serious clinical problem with inadequate treatment options ${ }^{90}$. CLI occurs in humans when an acute thrombus or heartderived embolus develops in the lower limb and causes a sudden decrease in perfusion that threatens limb viability. Cather-directed revascularization can treat marginally threatened limbs with recent occlusions and surgical revascularization is performed on acutely threatened limbs. Even with intervention, death and complication rates are high. Ten to fifteen percent of patients require limb amputation and fifteen to twenty percent die within one year of presentation. The primary predisposing pathology of CLI is peripheral artery disease (PAD), which is most commonly 
caused by atherosclerosis ${ }^{118}$. It affects around 10 million Americans. Most patients are elderly and experience intermittent leg pain and persistent soreness, and their disease can be exacerbated by smoking and diabetes. With incidences of obesity, diabetes, and cardiovascular disease increasing across the globe, more therapies to address critical limb ischemia are urgently needed.

The mouse hind limb ischemia model is directly correlated to human critical limb ischemia (CLI), and therefore has been a convenient platform to study the cellular, molecular and genetic mechanisms involved in the arteriogenic and angiogenic recovery of perfusion ${ }^{119}$. It is also a great platform to study new compounds with therapeutic potential and to examine potential benefits of RvD2 immunovascular modulation. In this model, the femoral artery and vein of the right hind limb is ligated and severed just inferior to the external iliac artery and deep to the inguinal ligament. The mouse is then allowed to recover over the course of two weeks to monitor restoration of blood perfusion to this damaged limb. Immediately after femoral artery and vein ligature, perfusion recovery in the upper mouse limb is predominantly due to arteriogenesis. A few days later, hypoxia in the lower limb stimulates angiogenesis, which becomes the primary avenue for perfusion recovery. Overall recovery by both processes can be monitored longitudinally by laser Doppler perfusion imaging (LDPI), which aims a laser at the limb surface and correlates flow-induced light scatter to quantitate perfusion ${ }^{120}$. Animals can be euthanized for histology, Microfil silicone pigment perfusion, and micro computed tomography (microCT) to examine gross vascular changes ${ }^{121}$. Therefore, we used the mouse hind limb ischemia model to evaluate the effects of the pro-resolving lipid mediator RvD2 on arteriogenic and angiogenic perfusion recovery following ischemia. LDPI was used to quantitate these effects, and we measured an increase in perfusion recovery by RvD2. We also evaluated overall vascular volume changes with Microfil vascular casting and microCT quantitation. Finally, we examined morphological changes in the limb muscle with hematoxylin \& eosin-stained paraffin histology 
sections $^{119}$. From these experiments we collected evidence that supports a beneficial role for proresolution RvD2 during revascularization after arterial occlusion.

\section{Experimental procedures}

Animals and reagents-Male $C 57 \mathrm{~B} / 6 \mathrm{~J}$ mice 8-10 weeks of age were purchased from Jackson Laboratories. All animal procedures were approved by the University of Louisville Institutional Animal Care and Use Committee (no. 11093). Resolvin D2 (RvD2) (7S,16R,17S-trihydroxy-4Z,8E, 10Z,12E,14E,19Z-docosahexaenoic acid) was purchased from Cayman Chemical (Ann Arbor, MI).

Hind limb ischemia model-We used two different models of ischemia, a severe model involving a more superior ligation site, and a more mild model involving a more inferior ligation site. For the severe model, the mouse subject was anesthetized on the surgical surface with $3 \%$ isoflurane in $2 \mathrm{~L} / \mathrm{min}$ oxygen. The right limb was then denuded by depilatory cream and sterilized by ethanol wipes. A $5 \mathrm{~mm}$ vertical skin incision was made lateral to the abdomen superficial to the inguinal ligament. The inguinal fat pad was separated from the peritoneal lining to reveal the proximal femoral artery branching from the external iliac artery. The femoral artery and vein were then separated from their protective sheath and two ligatures $2 \mathrm{~mm}$ apart were made. Vessels were then severed between the ligatures and the skin incision was closed with two discontinuous sutures and bonded with n-butyl-ester cyanoacrylate. For the mild model, mice were anesthetized and denuded. A $3 \mathrm{~mm}$ horizontal skin incision was made superficial to the medial right knee. The branch point where the femoral artery bifurcates into the saphenous artery and popliteal artery was exposed from its membrane sheath. The accompanying nerve was moved aside and a ligature was placed around the saphenous artery and vein immediately proximal to the femoral bifurcation. A second ligature was tied $1 \mathrm{~mm}$ below the first, and vessels between them were then 
severed. The skin was then closed with sutures and cyanoacrylate. Sham operated mice were opened, dissected, and closed, without vessel ligature. Twenty-four hours after surgery, mice were injected with either $0.9 \%$ saline vehicle $(100 \mu \mathrm{l})$ or resolvin D2 in vehicle (RvD2; $100 \mu \mathrm{l} ; 4 \mu \mathrm{g} / \mathrm{kg}$ body weight) subcutaneously just superficial to the ligation site.

Laser Doppler perfusion imaging-Mouse limbs analyzed by LDPI were fully denuded a day prior below the midline to allow the scanning laser to penetrate below the skin. The mice were then anesthetized with isoflurane and placed on its dorsal surface on a dark scanning surface above a $40^{\circ} \mathrm{C}$ heat pad. Perfusion of the ventral surface of both right and left hind limbs was measured with a PeriScan PIM 2 laser Doppler device (Perimed). Images were acquired one, seven and fourteen days after ligation. After acquisition, color-binned images of the mouse limbs were imported into ImageJ, and areas of blue, green and red in the region of interest (ROI) spanning the entire hind limb was enumerated. The value for the right limb was then normalized to the left (nonischemic) limb to calculate the \% recovery of blood perfusion.

Vascular casting and microCT-Mice were anticoagulated with $10 \mu \mathrm{L}$ intraperitoneal injection of 1000 units $/ \mathrm{mL}$ of heparin and sedated with $10 \mu \mathrm{L}$ of pentobarbital. Their hearts were then cannulated with a $15 \mathrm{~g}$ needle perfused systemically at $5 \mathrm{~mL} / \mathrm{min}$ with $10 \mathrm{~mL}$ of $100 \mu \mathrm{M}$ adenosine, $10 \mu \mathrm{M}$ nitroprusside and $0.05 \% \mathrm{wt} / \mathrm{vol}$ bovine serum album. Undiluted Microfil was mixed with hardening solution, perfused, and allowed to solidify. The entire lower body was de-skinned and placed in formalin for $24 \mathrm{~h}$ and Cal EX II for $48 \mathrm{~h}$ to fix and decalcify. Samples were then imaged with a MicroCAT II (Siemens) scanning at $80 \mathrm{kVp}$ and $500 \mu \mathrm{A}$, with $2 \times 2$ binning for a pixel resolution of $1024 \times 1024$ with $34 \mu \mathrm{M}$ voxel sizes. After thresholding of soft tissue, casted blood vessel voxel volumes were then quantified using Analyze (AnalyzeDirect). For whole-mount 
imaging of Microfil casted limbs, harvested limbs were places in successively higher solutions of glycerol $(40 \%, 60 \%, 80 \%, 100 \%$, solution changed every 24 hours) and imaged with a digital CCD camera (Nikon).

Histology-For histological analysis, mice were euthanized and their limbs were dissected. The upper limb semitendinosus, semimembranosus and biceps femoris (hamstring) muscles and the lower limb gastrocnemius muscle were excised and fixed in formalin and paraffin embedded. Three transverse cross sections of the muscles were mounted per slide and 2 slides were amassed per mouse. Gross examination of tissue injury was performed on hematoxylin and eosin (H\&E) stained sections. The number of myocytes with centrally located nuclei indicates muscle regeneration and this marker was used to assess the extent of limb damage and recovery with RvD2 treatment ${ }^{122}$. Five to ten low power fields were examined and the total number of myocytes and myocytes with centrally located nuclei were enumerated.

Data analysis, statistics-All reported data are reported as means \pm SEM. For all statistical analysis, Prism 6 (GraphPad) was used. Data were analyzed by one-way or two-way ANOVA followed Bonferroni post-tests for multiple groups, or unpaired two-tail Student's t test for direct comparisons. A $p$ value less than 0.05 was considered significant.

\section{$\underline{\text { Results }}$}

Limb perfusion is enhanced in ischemic limbs of mice treated with RvD2-To determine whether pro-resolving lipid mediators are capable of enhancing post-ischemia revascularization, we induced severe hind limb ischemia in eight week old male C57B/6J mice via ligature of their right femoral artery and vein, and then treated them with RvD2. The subcutaneous RvD2 
injections $(100 \mu \mathrm{L}$ volume, $4 \mu \mathrm{g} / \mathrm{kg})$ were commenced $24 \mathrm{~h}$ after surgery and continued daily for 14 days. We waited 24 hours for inflammation to develop in the ischemic limb and then introduced RvD2 on the first day post-surgery. The extent of perfusion recovery in RvD2-treated animals was identical to saline-vehicle treated control animals at $8 \%$ (Figure 1). By day 7, the recovery of RvD2treated animals had risen to $46 \%$ while control animals lingered at $20 \%$. At the last scan on Day 14 , the ischemic limbs of RvD2-treated animals had recovered $64 \%$ of original perfusion, while in control animal limbs perfusion was restored only to 29\%. Since RvD2-treated HLI-mice demonstrated an increased rate of perfusion recovery, the experiment was terminated at day 14 . Overall, the absolute increase in severe-model ischemic limb perfusion recovery in vehicle-treated mice, as measured by LDPI, is $21 \%(29 \%-8 \%)$, in RvD2-treated mice, it is $56 \%$ (64\%-8\%). From these data we concluded that RvD2 enhanced restoration of LDPI-measured blood flow in the mouse HLI model.

\section{RvD2 enhances arteriogenesis in mice undergoing HLI-To obtain a stereoscopic} understanding of RvD2-enhanced revascularization of mouse hind limb, we used Microfil, a radiocontrast-infused silicone elastomer, to cast the entire mouse vasculature and examine limb vascular volumes by microCT. We casted severe-HLI mice seven days after ligation and compared effects of vehicle versus RvD2 treatment. Due to the three-dimensional projection of voxels, a stereoscopic view of the entire lower mouse limb allowed us to visualize an extensive network of tortuous arteriogenic collaterals in the ischemic side of limbs. After quantitation of the entire limb voxels, we found that vehicle-treated mice were able to restore $89 \%$ blood perfusion in the ischemic limb compared with the nonischemic limb (Figure 2). RvD2-treated mice were able to restore $100 \%$ blood perfusion. We also casted mild-HLI mice three days after surgical occlusion and quantitated blood flow recovery. Vehicle treated mice recovered $69 \%$ of normal perfusion, 
while RvD2 treated mice recovered 92\% (Figure 3B). We also clarified the mild-HLI limb tissue to directly visualize the restoration of perfusion. Vehicle-treated mice limbs were not robustly perfused by Microfil and retained red coloration. RvD2-treated mice limbs were more extensively perfused by Microfil, and displayed pathognomonic signs of tortuous arteriogenic collateral arterioles (Figure $3 \mathrm{~A}$ ).

RvD2 increases number of regenerating myocytes-We performed histopathological analysis of mouse hind limb muscles to determine whether the extent of enhanced perfusion by RvD2 resulted in improved tissue repair. Necrotic muscle can be identified using $\mathrm{H} \& \mathrm{E}$ staining to highlight the presence of basophilic infiltrated leukocytes, hypercontracted myofibres, and degenerating myocytes with fragmented sarcoplasm. Regenerating muscle can be identified by the presence of small activated myoblasts with plump myofibres with centrally located nuclei. Since undamaged myocytes contain peripherally located nuclei, and dark blue hemalum-stained nuclei are easy to identify, we quantified the difference between RvD2-treated and vehicle-treated ischemic hind limbs by counting central versus peripheral nuclei in $\mathrm{H} \& \mathrm{E}$ sections of the hamstring muscles (Figure 4). We harvested limb muscles (hamstring) at two weeks after induction of ischemia, and found a higher percentage of regenerating myocytes in sections of RvD2-treated mice compared with vehicle-treated mice. As there was no ischemia in sham-operated animals, we did not find any centrally located nuclei in either vehicle-treated or RvD2-treated sham animals. Our overall histological analysis found qualitative evidence of reduced leukocyte infiltration and quantitative evidence of increased muscle regeneration. 
Figure 1: Laser Doppler perfusion imaging (LDPI) of mouse hind limb. Representative LDPI images and quantitation of vehicle or RvD2-treated mice at day 1, 7 and 14 after severe model of proximal femoral artery and vein ligation. The red box indicates ischemic region of interest (ROI). Percent recovery of blood flow is calculated as the number of red and green pixels in the ischemic ROI versus the nonischemic ROI. $n=8$ mice per group, ${ }^{\star} p<0.05$ 
Figure 1

Vehicle
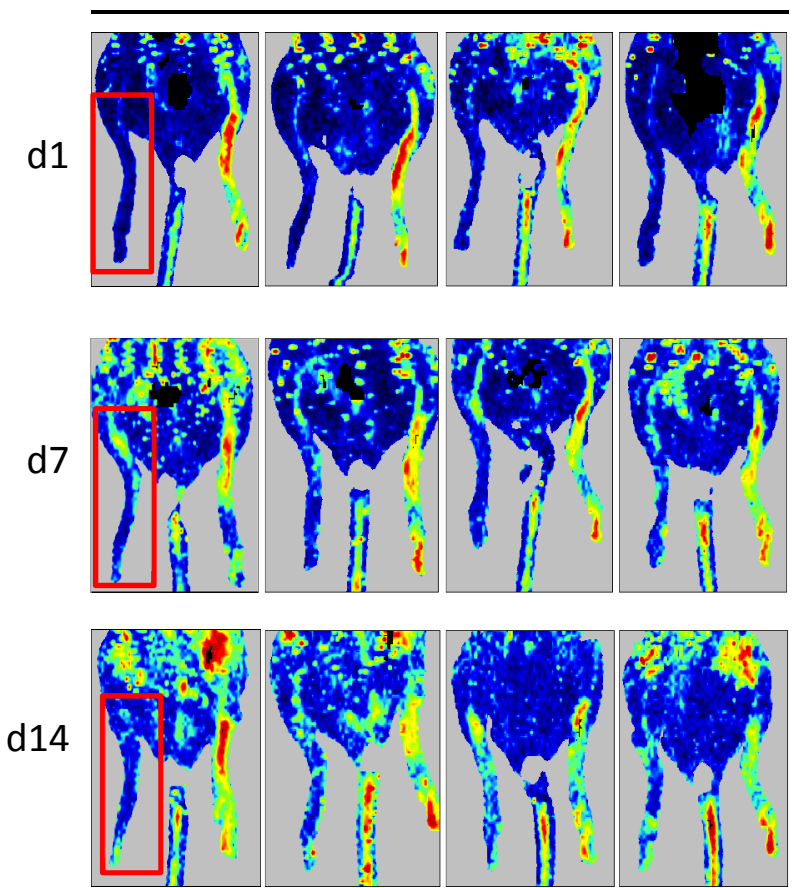

RvD2
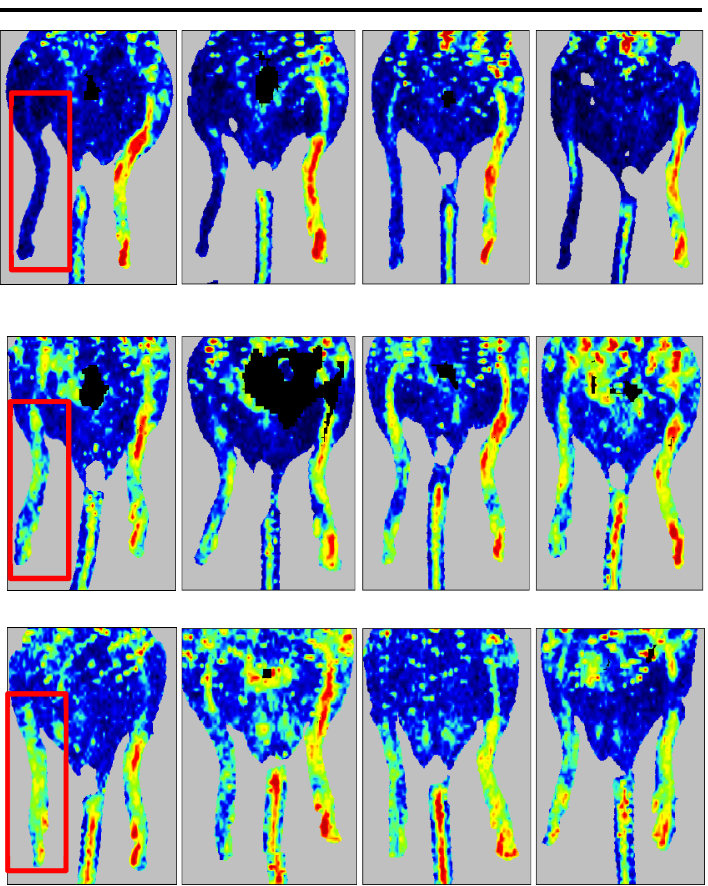

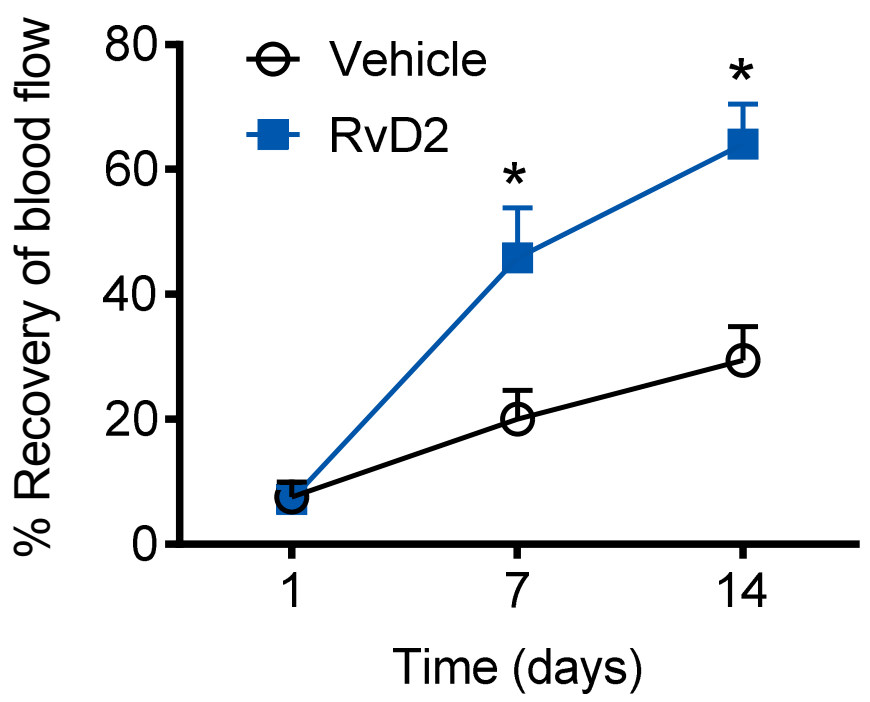


Figure 2: MicroCT of Microfil-casted limbs, severe HLI model. Representative microCT images and quantitation of Microfil-casted limbs of vehicle or RvD2-treated severe HLI model limbs 7 days after surgical ligation. Dotted line indicates superior site of ligation, arrows refer to tortuous arteriogenic arterioles. Some mice were lost to incomplete Microfil filling. $n=5-6$ mice per group, ${ }^{*} p<0.05$ 
Figure 2
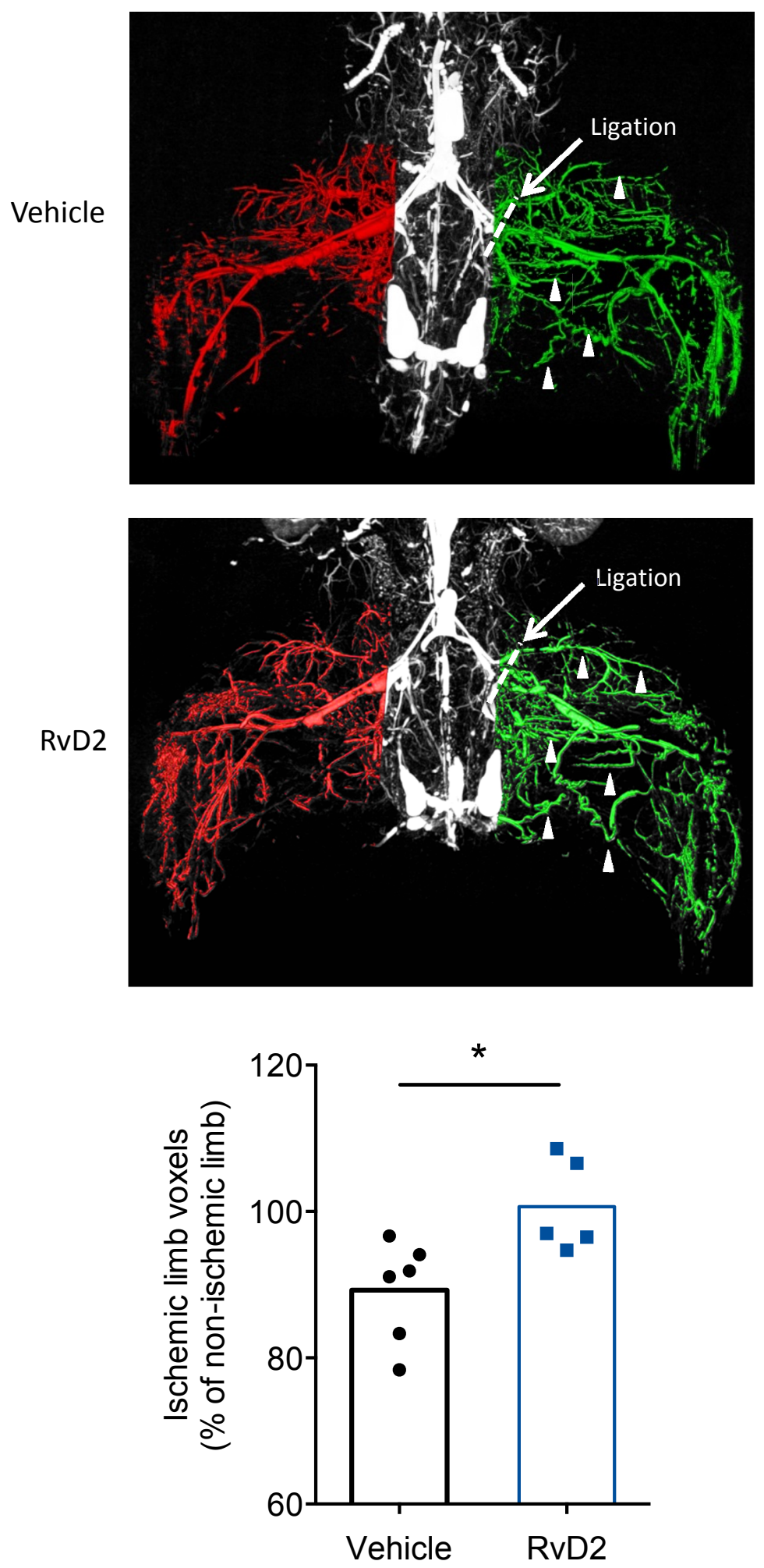
Figure 3: Whole mount and microCT images of Microfil-casted limbs, mild HLI model. (A) Representative whole mount images glycerol-clarified Microfil-casted limbs of vehicle or RvD2treated mild HLI model limbs 3 days after surgical ligation. Arrows refer to tortuous arteriogenic vessels. (B) Representative microCT images and quantitation of the same limbs. $n=7$ mice per group, ${ }^{*} p<0.05$ 
Figure 3

A.
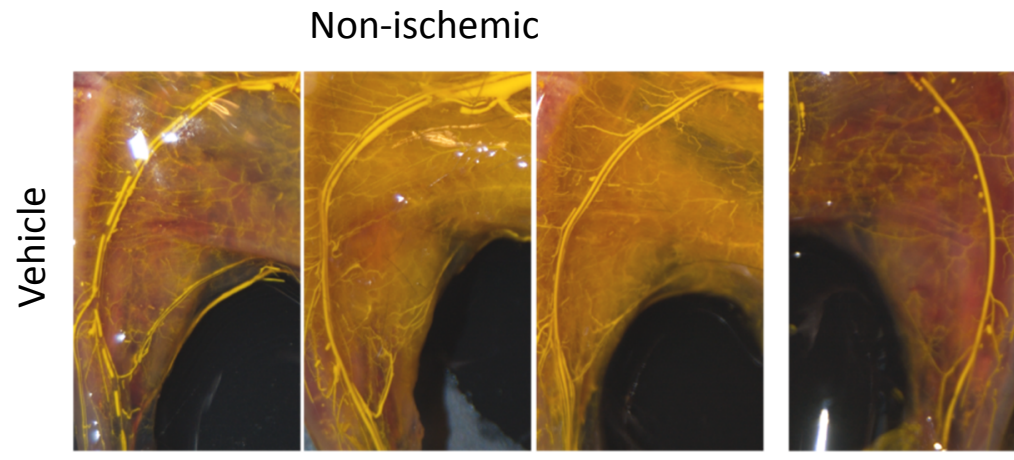

Ischemic
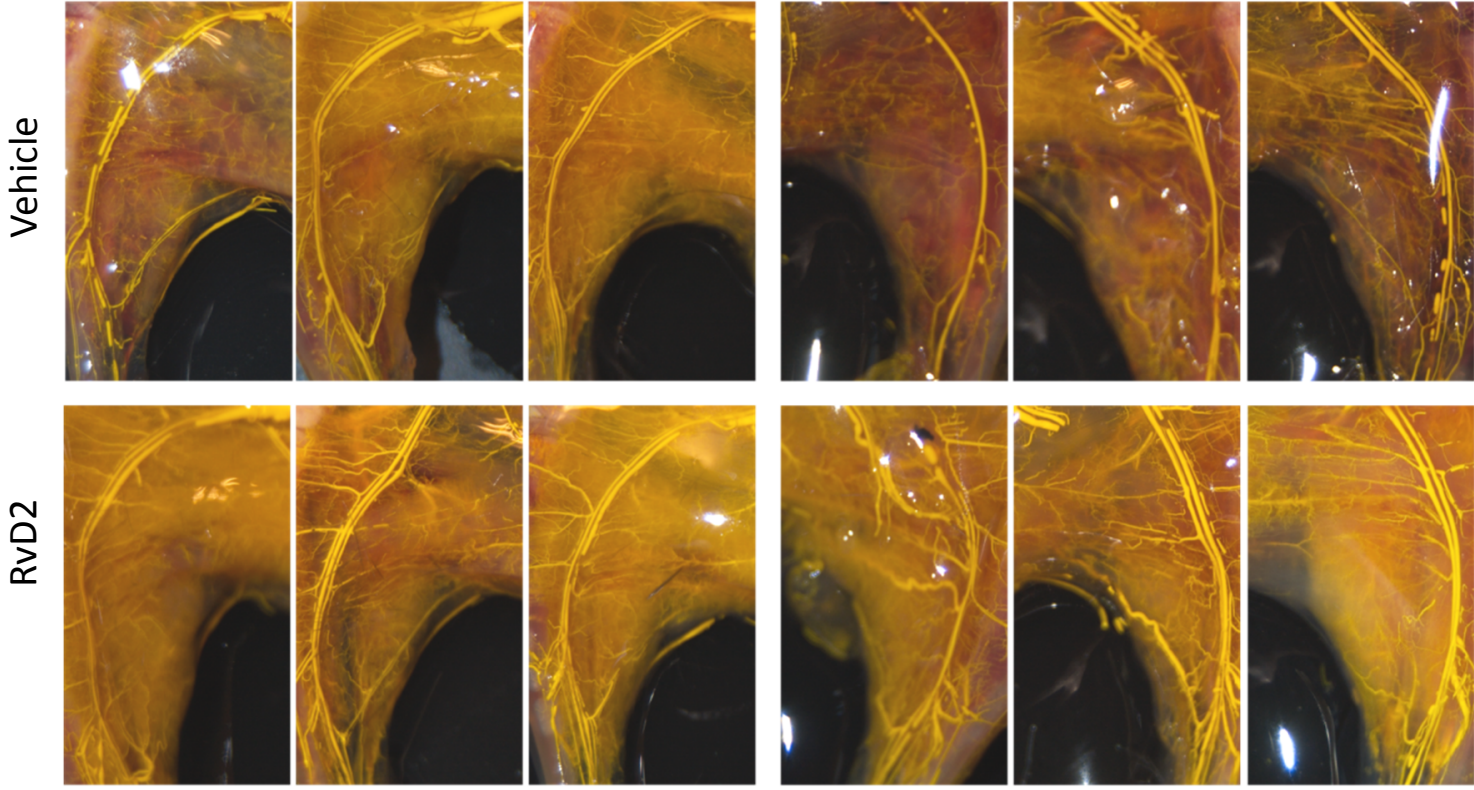

B.
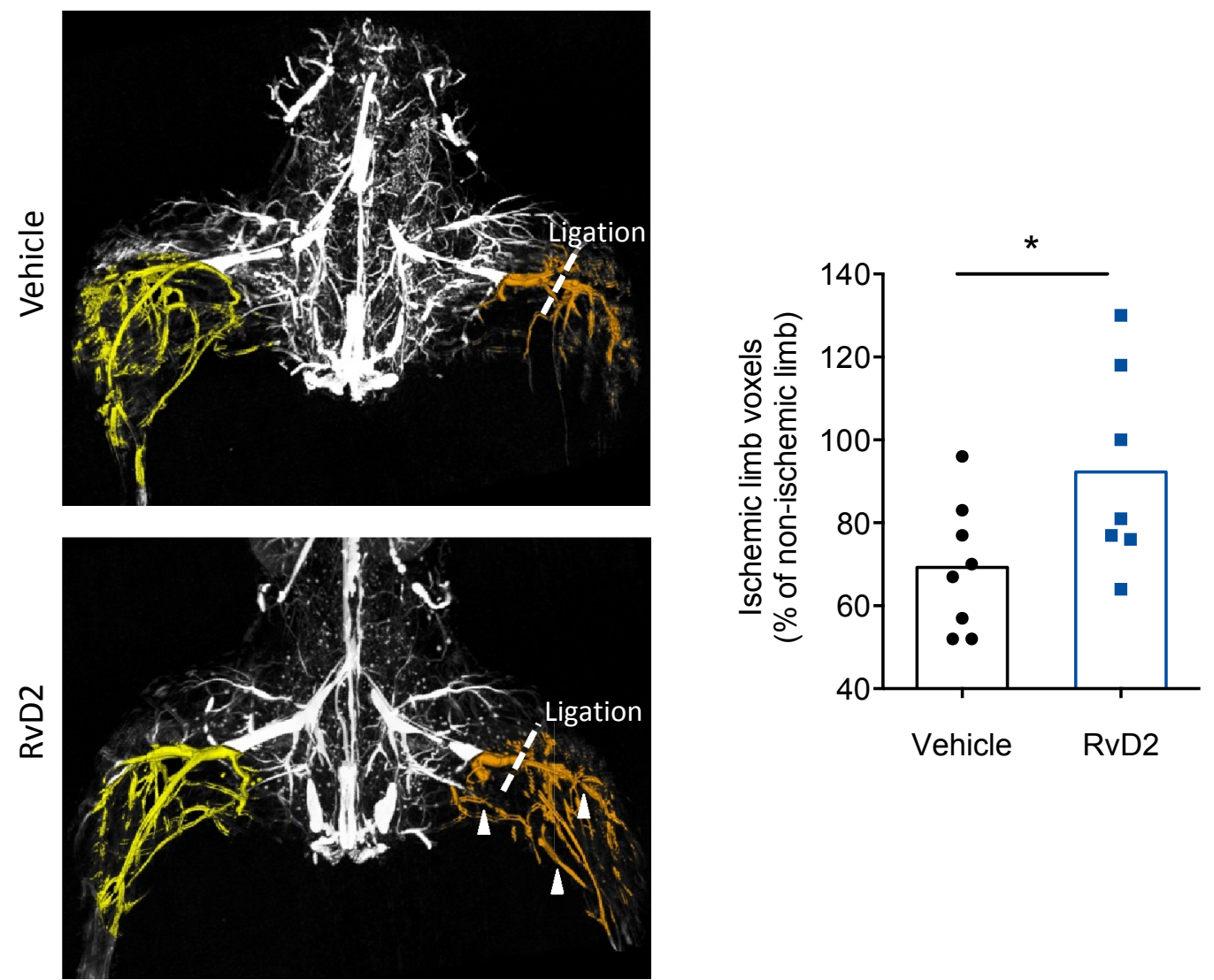
Figure 4: Histology of hamstring muscles, severe model of hind limb ischemia. Hematoxylin and eosin stained paraffin sections of vehicle and RvD2-treated right hamstring muscles fourteen days after severe model of HLI. Sham-surgery controls included for comparison. Regenerating myocytes with centrally located nuclei were enumerated as a percentage of total myocytes. Quantitation was based on five to ten high power fields per group. ${ }^{*} p<0.05$ 
Figure 4
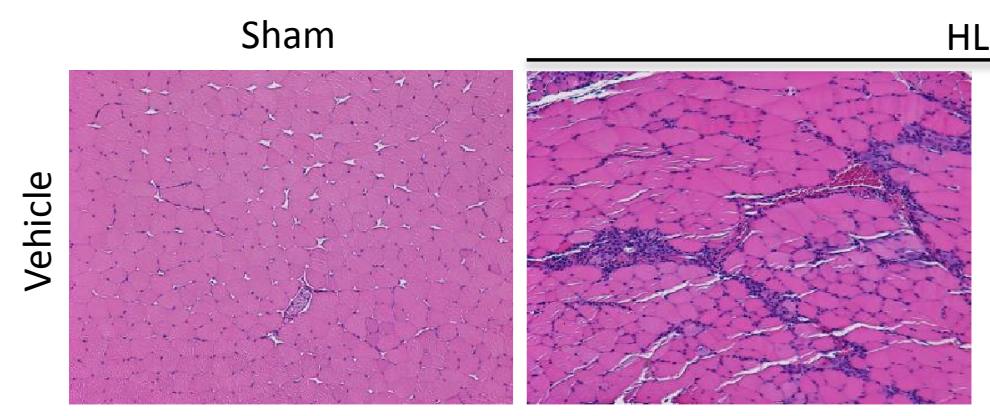

HLI
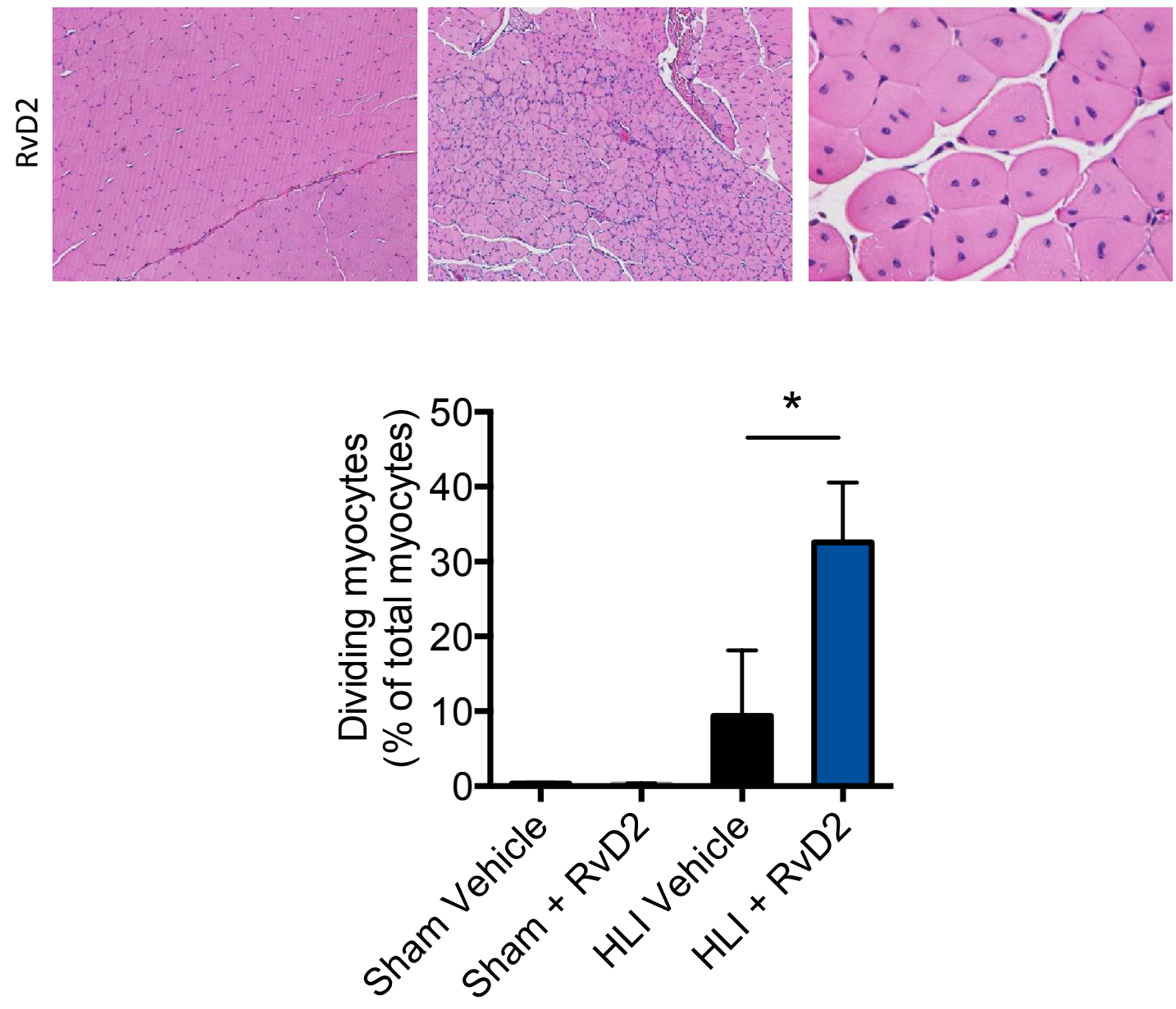


\section{Discussion}

In this study, we have provided evidence that pro-resolving lipid mediator resolvin D2 can enhance the blood perfusion recovery of femoral artery and vein-occluded ischemic mouse hind limbs. We report longitudinal data over a two week period using LDPI and endpoint data at seven days using Microfil-microCT with a severe model of hind limb ischemia. We also provide histologic evidence of RvD2-stimulated muscle regeneration in the hamstring muscles, and corroborated RvD2 efficacy in a more mild model of hind limb ischemia.

We found that RvD2 in highly effective in enhancing post-ischemia revascularization, and its efficacy compares very favorably with other reported therapeutic strategies. LDPI is the gold standard upon which all mouse HLI studies are quantitated ${ }^{119}$. In our severe HLI model, we obtained a $56 \%$ absolute increase in ischemic limb perfusion after 14 days. In a study by other investigators, daily administration of plasminogen activator inhibitor-1 after ischemia induction produced an absolute perfusion recovery of $25 \%$. In another study, an infusion of endothelial progenitor cells (EPCs) 3-6 hours after ischemia improved perfusion by $30 \%$ fourteen days later ${ }^{123}$. In another study, the intramuscular electroporation of a human VEGF-containing plasmid increased 14 -day limb perfusion by $35 \%^{124}$. Combining the VEGF plasmid with a hepatocyte growth factor (HGF)-containing plasmid yielded a perfusion increase of $50 \%$ at day 14 . Finally, in a BMX-kinase transgenic mouse, increased Tie-2 promoter-driven overexpression of BMX promoted limb perfusion recovery by $80 \%$ at day $14^{125}$. While impressive, these other studies have major caveats for therapeutic translation. The BMX kinase study produced impressive results, but there are currently no drugs that approximate the transgenic mouse model by stimulating BMX kinase overexpression. VEGF and other growth factor therapies have also proven auspicious in preclinical studies, but in clinical trials no consistently efficacious therapeutic program has been established. Cell therapy, such as with EPCs, or monocyte and macrophage-derived cocktails, have 
shown promise in pre-clinical studies, and the macrophage cocktail Ixmyelocel- $T$ is currently progressing in phase 3 clinical trials, but the overall process treatment process is lengthy (months) and the cocktail is not chemically defined.

In comparison with other interventions, RvD2 possesses many advantages as a therapeutic and pharmaceutical candidate. RvD2-therapy does not involve any germline modifications. The absolute efficacy is higher than both EPC infusion and growth factor therapy. Also, RvD2 is a singular endogenous compound that can be synthesized artificially by total organic synthesis, and is therefore chemically defined. Finally, RvD2 is efficacious in enhancing perfusion recovery in a clinically feasible and therapeutically relevant manner, because we could observe a beneficial effect even when administered $24 \mathrm{~h}$ after initial ischemia. Patients presenting with coronary artery occlusion or critical limb ischemia would not need to be preemptively treated with RvD2 for therapeutic effect. Although given the other beneficial effects of pro-resolving lipid mediators in diabetes and $\omega-3$ supplementation, perhaps preemptive pro-resolution therapy may be a good strategy. Nevertheless, many important translational questions about the use of RvD2 remain. For instance, what is the optimal dose of RvD2? Are daily injections necessary? Can RvD2 simply be administered topically? Given the promising evidence we have presented in this study, these translational questions can be addressed in the future.

The Microfil casting and micro-computed tomography of revascularizing mouse limbs is a rigorous and holistic evaluation of perfusion recovery. One of the limitations of LDPI is the inability to penetrate deep into tissues, thus it can only measure blood flow in vessels that are near the surface of the $\mathrm{skin}^{126}$. The femoral artery is sufficiently superficial to be imaged by LDPI, but other arterioles and developing collaterals deeper in the muscles cannot be imaged. This is especially important in our severe HLI model, as our superior femoral ligation site disrupts blood flow to the entire hind limb. In other studies, the femoral artery and vein ligation site is more 
inferior and thus only disrupts perfusion to lower limb and foot. Regardless of ligation site, Microfil casting of the limb vasculature is a versatile procedure. Once casted, limbs can be clarified with glycerol and blood vessels can be whole mounted and photographed. This allows investigators to appreciate visually the appearance of tortuous arterioles, which are hallmarks of arteriogenesis. Some studies have directly measured arteriole diameter in whole mount images, but this again captures only the surface vessels. Fortunately, the lead chromate radioconstrast in Microfil allows computed tomography to obtain a stereoscopic image of the entire limb vasculature. Initially we experienced difficulty distinguishing Microfilled blood vessels and mouse bone. This problem was addressed by decalcifying the bone with formic acid (CalEX II) and using more concentrated Microfil ${ }^{121}$. With additional thresholding of CT image intensities, we were able to obtain detailed images of the entire limb vascular tree and objectively quantitate the voxel volumes of nonischemic limb versus the ischemic limb using the powerful Analyze software. Therefore, rather than capturing only surface blood flow by LDPI, or measuring only select vessel diameters by micrograph, we quantified the entire limb vascular volume to rigorously and holistically evaluate RvD2 efficacy.

There are some weaknesses to the Microfil-microCT method. Microfil is infused as viscous fluid that hardens inside blood vessels after 30 min. However, the perfusion of Microfil through the vasculature is a stochastic process that is difficult to control. There is leakage of Microfil at the left ventricle infusion site, and the filling of large veins is often incomplete, leaving discontinuous sections of hardened Microfil. Additionally, arteriovenous anastomoses inhibit the ability to fill only outbound blood vessels, but not inbound vessels. The incomplete filling of some vessels and disproportionate filling of the large femoral veins was particularly troublesome during quantitation of three-dimensional microCT images, as visually appreciable differences in perfusion recovery were obfuscated by artifacts of Microfil infusion. This was the primary impetus to 
perform the mild model of hind limb ischemia. The lower ligation site allowed us to focus on a more distal portion of the limb vasculature to circumvent variations in large vein casting. Naturally, the less severe ischemia model results in faster perfusion recovery and thus we performed our casting-imaging procedure at an earlier time point to capture the effects of RvD2. Although this mild model addressed certain study limitations, the major limitation of MicrofilmicroCT remained: resolution and detection limits impeded the ability to accurately measure capillary volume. Because increases in capillary volume are the primary contributions of angiogenesis to revascularization, our inability to measure capillary volume also precluded our ability to quantitate angiogenesis in the limbs. The resolution of microCT is very high (it can resolve 10 microns) but the primary handicap is the Microfil reagent ${ }^{127}$. Its high viscosity hinders capillary perfusion and its modest radiocontrast produces dim images that are difficult to distinguish from background. In the future, improved vascular casting and radiocontrast agents can be used to fill capillaries and allow direct measurement of angiogenesis.

We performed only limited histology in our study. Other investigators stain limb muscle sections for leukocyte infiltrates and endothelial cells to quantitate tissue recovery after ischemia ${ }^{119}$. Although these methods are established and rigorous, our Microfil-microCT and flow cytometry (Chapter 4) methods are more objective and capture a larger sample size. We show a few representative histology images and performed rudimentary muscle regeneration analysis primarily to corroborate the other methods. We did not perform any functional testing or neurological status of the mouse limbs. Our study was focused on evaluating the extent of perfusion recovery. However, recovery of blood flow may not be directly proportional to restoration of function. Visually, RvD2-treated mice appear to have a larger range of motion than vehicle-treated mice as early as day 2 (data not shown). This is a subjective observation and in the future mice can be subject to some type of exercise (treadmill, swim tank) to quantify the 
functional status of their ischemic limb. Furthermore, CLI patients often experience pain in their $\operatorname{limbs}^{90}$. Other resolvins have already demonstrated direct inhibitory action on pain receptors. In the future, neurological testing on RvD2-treated mice limbs can confirm if RvD2 can also alleviate pain after ischemia. Coupled with its perfusion enhancing effects, any potential analgesic effect will only further enhance therapeutic efficacy.

Finally, there are some limitations to the hind limb ischemia model in general. Although femoral artery ligation is anatomically relevant to human critical limb ischemia $(C L I)^{128}$, the etiology and the severity are not. The most common cause of CLI, peripheral artery disease (PAD), was not a component of our HLI model and the mice in both of our HLI models eventually recovery full limb perfusion ${ }^{90}$. Outcomes of our mice models do not correspond to the mortality of CLI patients. The primary reason is that CLI patients are older and suffer other comorbidities whereas the mice we used were young and healthy. Other investigations with diabetic mice have demonstrated a revascularization defect like humans ${ }^{129}$. Therefore, to assess the efficacy of RvD2 treatment more closely to humans, it will be important to evaluate the extent of perfusion recovery by RvD2 in mice with atherosclerosis or diabetes.

In summary, we have found in this study that RvD2 improves ischemic mouse hind limb blood flow recovery. We provide quantitative evidence by LDPI, Microfil-microCT, and histology. The efficacy of RvD2 compares favorably to other therapeutic candidates. We were unable to quantitate specifically angiogenesis and did not measure functional recovery. More studies will be necessary in the future to address these limitations and also examine a more clinically relevant diseased mouse model. 


\section{CHAPTER III}

\section{MONOCYTES PRODUCE RESOLVIN D2}

\section{Introduction}

Found in all living systems, polyunsaturated fatty acids display extraordinary diversity. Although many organisms possess desaturases to generate PUFAs de novo, most animals do not possess this ability and therefore must obtain PUFAs from their $\operatorname{diet}^{31}$. Although this need limits the PUFA heterogeneity in animals to mostly $\omega-6$ and $\omega-3$ PUFAs, their cis-configured double bonds allow the formation of a motely portfolio of peroxide, epoxide and hydroxide metabolites. Furthermore, animals have evolved complex enzyme systems to synthesize this wide collection of PUFA metabolites in a stepwise manner and employ them in a multiplicity of signaling tasks.

This process begins with the incorporation of PUFAs into plasma membrane phospholipids and then their subsequent liberation upon stimulation. The incorporation of PUFAs into the plasma membrane phospholipids phosphatidylcholine, phosphatidylserine, phosphatidylinositol, and phosphatidylethanolamine, requires two enzymatic steps within the interstitium of membrane ${ }^{31}$. First, the acyl-CoA synthetases form PUFA-CoA from free nonesterified PUFA. Then, various lysophosphatide acyltransferases attach the PUFA-CoA to the sn-2 position of phospholipids. The relative abundance of these PUFAs in the plasma membrane, mainly arachidonic acid (AA), eicosapentaenoic acid (EPA), and docosahexaenoic acid (DHA) differs between cells and organs. AA can comprise up to $25 \%$ of all fatty acids in T-cell membranes, whereas DHA is especially enriched in the brain ${ }^{130}$. Although the PUFA content can affect the general physical characteristics of the plasma membrane; many studies suggest that 
PUFAs cluster in signaling islands or lipid rafts. However, the signaling functions of these lipids begin after their liberation from the phospholipid backbone by the phospholipase A2 (PLA $)$ family of enzymes ${ }^{131}$. There are here many families of PLA $A_{2}$ enzymes: intracellular CPLA $A_{2}$ and $i P L A_{2}$ families and the extracellular $\mathrm{SPLA}_{2}$ family. The main role of $\mathrm{CPLA} 2$ is initiation of PUFA metabolism, the main role of iPLA2 is maintaining membrane homeostasis, and the main role of sPLA2 is modulating extracellular phospholipids. Group IVA CPLA 2 is the prototypic PLA 2 it is the central regulator of PUFA release from the sn-2 position of membrane phospholipids. The gene encoding this lipase is located on chromosome $1 q 25$, next to the COX 2 gene. While $\operatorname{CPLA}_{2} \alpha$ is constitutively expressed in most cells and tissues, inflammatory mediators or reparative growth factors can regulate $\mathrm{CPLA}_{2} \alpha$ gene translation and protein phosphorylation ${ }^{132}$. After expression, $\mathrm{CPLA}_{2} \alpha$ remains in the cytoplasm until an intracellular influx of calcium increases its affinity for specific membrane phospholipids by interacting with the $\mathrm{C} 2$ domain of the protein. This results in the translocation of the enzyme to the plasma membrane where it catalyzes the liberation of PUFAs esterified to phospholipids.

The PUFAs liberated from the membrane are metabolized by various enzymes, including the cyclooxygenases, lipoxygenases and cytochrome P-450 monooxygenases ${ }^{133}$. Of these, the cyclooxygenase-prostaglandin pathway was the first to be discovered. The cyclooxygenases (COX1 and COX2) are bifunctional enzymes that convert AA (and other PUFAs) to prostaglandin $\mathrm{H}_{2}\left(\mathrm{PGH}_{2}\right)^{134}$. While COX1 is constitutively expressed on most cells, COX2 is induced by several inflammatory mediators and is expressed in a limited number of cell types. These enzymes catalyze cyclooxygenation of the lipid, which is followed by a second heme-dependent peroxidation reaction. In these reactions, the 13-pro-S hydrogen is first stereospecifically removed from AA to yield a C11-C15 delocalized pentadienyl radical, which then reacts sequentially with two molecules of $\mathrm{O}_{2}$ to form the bicyclic hydroperoxy endoperoxide prostaglandin $G_{2}\left(P_{G} G_{2}\right)$. 
Next, the 15-hydroperoxyl group of $\mathrm{PGG}_{2}$ is reduced to yield $\mathrm{PGH}_{2}$. This prostaglandin is the common intermediate from which all the bioactive 2-series prostaglandins are derived. Although is $\mathrm{PGH}_{2}$ generated in many different cell types, the further oxidation of $\mathrm{PGH}_{2}$ by specific synthases such as, PGE synthase, PGD synthase, PGF synthase, PGI synthase, and TXA synthase, is more celltype specific ${ }^{133}$. For example, platelets contain TXA synthase and synthesize $\mathrm{TXA}_{2}$ from $\mathrm{PGH}_{2}$ to stimulate vasoconstriction and platelet aggregation during hemostasis. On the other hand, endothelial cells express PGI synthase and biosynthesize $\mathrm{PGI}_{2}$ and renal collecting tubule cells contain PGE synthase and produce $\mathrm{PGE}_{2}$. Thus the formation of COX-derived products is the first step in prostaglandin metabolism, and these products are used by various cell-specific synthases to generate various prostaglandin end products.

PUFAs are also metabolized by the lipoxygenase family of enzymes. These enzymes catalyze the oxygenation of PUFAs at specific unsaturated carbons and are named according to the position of the carbon atom oxygenated ${ }^{133}$. To-date, three types of lipoxygenases (LO) have been described in mammals: 5-LOX, 12-LOX and 15-LOX. They are expressed in leukocytes, platelets, reticulocytes and different types of epithelial cells. One problem with the atom-position designation of LOX activity is the functional overlap of oxygenation activity. For example, 15-LOX and 12-LOX can both catalyze the oxygenation of linoleic acid to 13-hydroperoxyoctadecadienoic acid. Also, soybean LOX-1 can add oxygen to both C5 and C8 of 15hydroxyperoxyeicosatetraenoic acid $(15-\mathrm{HPETE})^{31}$. Thus lipoxygenase oxygenation site is substrate-dependent and not enzyme-dependent. As a result a small number of enzymes can generate a large variety of oxygenated PUFAs: humans encode six functional LOX genes; mice encode seven. Mice do not encode a reticulocyte-type 15-LOX like humans, but produce a leukocyte 12-LOX (termed 12/15-LOX) that shares nearly 40\% sequence homology and performs similar functions. 
In humans and mice, lipoxygenases can generate both leukotriene B4 (LTB4) and lipoxin A4 (LXA4) $)^{31}$. The first step in their biosynthesis involves the oxygenation of AA at C5 to 5-HPETE, and then dehydration of the peroxide to from leukotriene A4 (LTA4), a highly unstable conjugated triene epoxide, which is an intermediate common to bothLTB4 and LXA4 synthesis. The LTA4 is then converted to LTB4 by LTA4 hydrolase; whereas the conversion of LTA4 to LXA4 is catalyzed by 15-LOX. This divergence is highly significant during the course of inflammation because LTB4 and LXA4 have opposing actions. LTB4 is pro-inflammatory; it is a potent neutrophil chemoattractant, whereas, LXA4 is anti-inflammatory and it inhibits neutrophil infiltration. During the early phases of inflammation, the LTB4 substrate LTA4 is predominantly shuttled through LTA4 hydrolase to promote inflammation. However as the timecourse of inflammation shifts to resolution, LTA4 is diverted to 15-LOX to simultaneously increase LXA4 synthesis and decrease LTB4 production. This diversion of substrate and switch in lipid mediator class is an important transition signal during the course of inflammation and wound healing. Furthermore, lipid mediators do not have be synthesized in toto in a single cell. For example, mast cells can generate LTA4 and release it into the blood where it is absorbed by reticulocytes and processed to LTB4 ${ }^{135}$. This process of transcellular biosynthesis adds another level of lipid mediator regulation. It is these different levels of regulation and the large number of different AA-derived lipoxygenase products that contributes to the complexity of PUFA metabolism. Furthermore, DHA and EPA are also prominent PUFAs in animals, and they can be oxygenated by lipoxygenase enzymes. The same enzymes that generate LTB4 can synthesize LTB5 from EPA, which is only structurally different from LTB4 at the $\omega-3$ bond position ${ }^{108}$. Lipoxygenases can also synthesize oxygenation products from DHA; however due to its extra two carbons and double bond, DHA-LOX products are more structurally distinct.

Resolvin D2 (RvD2) is a recently discovered lipid mediator synthesized by lipoxygenases 
from $D H A^{55}$. RvD2 was first discovered in nanogram amounts during the resolution phase exudates of a mouse dorsal air pouch TNFo-induced inflammation model. It is a potent bioactive lipid mediator capable of decreasing leukocyte-endothelial interactions during inflammation, but also stimulate macrophage phagocytosis and endothelial NO production. In vivo, the biosynthesis of RvD2 first requires the generation of 17-hydroxyperoxydocosahexaenoic acid (17-HpDHA) by 15-LOX oxygenation of C17 on DHA. The 17-HpDHA is then transformed into a 7(8)-epoxidecontaining intermediate by 5-LOX and finally enzymatically converted to RvD2. This synthetic process can occur in a single cell or through multiple cells. Neutrophils produce both 15-LOX and 5-LOX and can produce RvD2 from DHA per se or from 17-HpDHA precursor by transcellular synthesis after interacting with other 15-LOX expressing cells. Eosinophils express high levels of 15-LOX but not 5-LOX. The 15-LOX converts DHA to 17-HpDHA which diffuses to neutrophils that then produce more RvD2. There is also evidence that macrophages can produce RvD2. Mouse peritoneal macrophages express both 12/15 lipoxygenase and 5-lipoxygenase ${ }^{136}$, and macrophages derived from human peripheral blood monocytes can produce RvD2 in vivo ${ }^{137}$. Overall RvD2 is a novel DHA-derived lipid mediator that can be synthesized by lipoxygenases in leukocytes.

In this study, we sought to determine if during revascularization, a specific cell type is capable of RvD2 synthesis. We verified the activity of 12/15-lipoxygenase in the hind limb after femoral artery occlusion, determined the leukocyte populations that are most prominent during revascularization, and measured their ability to synthesize RvD2. We first measured the overall production of lipid mediators in the muscle by targeted lipidomics analysis, and found elevated levels of 12/15-lipoxygenase metabolism products. We identify the different leukocyte populations in the ischemic hind limb by digesting the muscle tissue and analyzing the cells by flow cytometry. Monocytes, macrophages, and neutrophil populations, but not lymphocytes were 
increased during HLI. In addition, we isolated Ly $6 \mathrm{C}^{\text {hi }} \mathrm{CCR} 2^{+}$inflammatory monocytes from mouse spleen and assess their ability to synthesize RvD2 after in vitro stimulation. Taken together, our data suggest that during post-ischemia revascularization the lipid mediator RvD2 was produced by inflammatory monocytes.

\section{Experimental procedures}

Solid phase extraction and LC/MS/MS MRM detection of lipid mediators-Pulverized limb muscle tissue or ionophore-stimulated monocytes were frozen in ice cold methanol at $-80^{\circ} \mathrm{C}$ one day prior to solid phase extraction. For quantification the samples were spiked with lipid standards (d8-5HETE, d4-PGE2, and d5-RvD2, 2ng each) to calibrate recovery during extraction. Butylated hydroxytoluene was added to prevent non-enzymatic oxidation. Lipid mediators were then purified by solid-phase extraction on a reverse phase C18 column. Methyl formate-eluted fractions were collected, reduced in volume by nitrogen gas evaporation and reconstituted in methanol. These samples were then analyzed by liquid chromatography-tandem mass spectrometry (LC-MS/MS) using a C18 column $(4.6 \mathrm{~mm} \times 50 \mathrm{~mm} \times 5.0 \mu \mathrm{M})$ equipped high performance liquid chromatography system (HPLC; Shimadzu) coupled to a triple quadrupole mass spectrometer (API 2000; Applied Biosystems) operated in negative ionization mode. Samples were eluted with a mobile phase consisting of water:acetic acid (100:0.1 vol/vol) and acetonitrile:methanol (4:1 vol/vol) (73:27) and ramped to 30:70 over $11 \mathrm{~min}$, followed by slowly ramping up to $20: 80$ over the next $10 \mathrm{~min}$ and held for additional 2 min. Finally, the mobile phase was ramped up to and held at 0:100 for $2 \mathrm{~min}$, followed by a return to 73:27 for 2 min. Entire sample elution was performed using a constant flow rate of $300 \mu \mathrm{L} / \mathrm{min}$. Unesterified free $A \mathrm{~A}$, EPA, and DHA and its endogenous metabolites were identified by multiple-reaction monitoring using established ion pairs. Quantitation of metabolites was performed using external calibration 
curves developed for each metabolite using purified standards (Cayman Chemical). Total extraction recovery and original tissue amount was calculated relative to deuterium-labelled internal standard and the original tissue weight or cell count.

Flow cytometry of digested limbs-To identify the different individual cell populations of the upper hind limb, three-day post-ischemia mice were sedated with pentobarbital and exsanguinated by cardiac puncture. The right semitendinosus, semimembranosus and biceps femoris (hamstring) muscles of the upper limb were then excised, weighed, and placed in enzyme digestion solution consisting of $500 \mathrm{U} / \mathrm{mL}$ collagenase II and $2.5 \mathrm{U} / \mathrm{mL}$ dispase II in $1800 \mu \mathrm{L}$ of Hank's Balanced Salt Solution. Muscles were left to digest at $37^{\circ} \mathrm{C}$ for $1.5 \mathrm{~h}$. The digestate was then strained through a $40 \mu \mathrm{M}$ cell strainer and washed with $2 \mathrm{~mL}$ of phosphate buffered saline with $1 \%$ bovine serum albumin (PBS-BSA). The strained cells were then stained with Fc-block (anti-CD16/32) and then stained with either a monocyte panel or lymphocyte panel of antibodies. The monocyte panel consisted of FITC anti-CCR2, PE anti-Siglec-F, APC anti-F4/80, APC/Cy7 antiLy-6G, BV421 anti-CD11b, BV605 anti-Ly-6C, and PerCP anti-CD45. The lymphocyte panel consisted of FITC anti- $\alpha$ V 3 , APC anti-CD8, PE anti-CD19, Pacific Bleu anti-CD4, and PerCP antiCD45. After 30 min of staining, cells were washed with $1 \mathrm{~mL}$ of PBS-BSA and then enumerated on a BD LSR II flow cytometer.

Splenic monocyte isolation and in vitro stimulation-To obtain the splenic population of inflammatory monocytes, intact spleens were rapidly excised from asphyxiated mice and ruptured between two microscope slides. The spleen was then rubbed through a $40 \mu \mathrm{M}$ cell strainer and rinsed with Pharmlyse to lyse red blood cells. After a 5 min incubation in the dark at room temperature, the strained cells were washed with phosphate buffered saline containing $2 \mathrm{mM}$ 
EDTA and $0.5 \%$ bovine serum albumin (MACS buffer). A Mouse Monocyte Enrichment Kit from Stemcell Technologies was then used to deplete the non-monocyte populations by antibodybiotin-magnetic bead separation. The remaining non-depleted population was confirmed by flow cytometry to be approximately $95 \%$ pure $\mathrm{Ly}^{-6 \mathrm{C}^{+}} \mathrm{CCR} 2^{+}$monocytes. In $12 / 15$-lipoxygenase knockout mice, additional biotin-conjugated ant-F4/80 antibody was added to the enrichment procedure to deplete an extra population of macrophages. After isolation of splenic monocytes, cells were washed with Hank's Balanced Salt Solution to remove EDTA and supplement calcium. Monocytes were then divided and stimulated with $10 \mu \mathrm{M}$ of $\mathrm{A} 23187$ calcium ionophore and $10 \mu \mathrm{M}$ docosahexaenoic acid for 30 min at $37^{\circ} \mathrm{C}$. The stimulation was then halted with $1.5 \mathrm{~mL}$ of ice cold methanol and the entire solution was frozen at $-80^{\circ} \mathrm{C}$ for LC-MS/MS analysis.

\section{Results}

$12 / 15$ lipoxygenase is active during revascularization-12/15-Lipoxygenase (12/15-LOX) is a lipid-peroxidating enzyme expressed in reticulocytes, eosinophils, airway epithelial cells, monocytes and macrophages. It is also the first biosynthetic enzyme in RvD2 production and it converts DHA into 17-HpDHA (Figure 5A). Therefore 12/15-LOX must be expressed and active to generate RvD2 during post-ischemia revascularization. Therefore to determine $12 / 15$-LOX in the post-ischemic limb, we harvested mouse ischemic limb tissue three days after right femoral artery and vein ligation. The tissue was pulverized, unesterified lipids were extracted by solid phase extraction, and the various lipid mediators were identified by LC-MS/MS (Figure 5B). This analysis led to the identification of the well-described inflammatory mediators LTB4, PGE2 and PGD2. Detectable levels of these lipids confirms that our procedure for inducing hind limb ischemia caused tissue damage and elicited a reparative response involving inflammation. We also found 5-HETE and 4-HDHA, as well as LTB4, which suggests the presence of an active 5-lipoxygenase 
biosynthetic pathway. More importantly, we detected the 12/15-LOX metabolites $12-\mathrm{HETE}$, 15HETE, 12-HEPE, 15-HEPE, 14-HDHA, and 17-HDHA in extracted muscle tissue, indicating the presence of 12/15-LOX activity. The amount of 12/15-LOX products was several fold higher than 5-LOX products, suggesting the overall activity of 12/15-LOX is higher than 5-LOX. Furthermore, because the HETEs are metabolites of AA, the HEPEs are metabolites of EPA, and the HDHAs are metabolites of DHA, their simultaneous presence implies that cells involved in revascularization are utilizing all three predominant PUFA substrates (AA, EPA, DHA). Interestingly, the production of DHA metabolites was several-fold higher than their EPA and AA counterparts. Overall, these results suggest hind limb ischemia elicits the production of established inflammatory lipid mediators and is associated with increased synthesis of 5-LOX and 12/15-LOX metabolites.

\section{Ly-6C ${ }^{\text {hi }}$, CCR2 $^{+}$inflammatory monocytes are elevated during revascularization-Ly- $6 C^{\text {hi }}$,} $\mathrm{CCR}^{+}$inflammatory monocytes (referred herein as inflammatory monocytes) are critical to the revascularization process. Therefore, we analyzed mouse hind limbs to determine if these cells are recruited in our revascularization model. For this, we digested limb tissue from sham control and femoral-ligated animals with collagenases, fluorescently labelled the liberated cells with a panel of leukocyte marker antibodies, and measured cell numbers by flow cytometry. We isolated tissue three days after ligation surgery to correspond with our previous lipidomics data. In this three-day post-ligation tissue, we found an elevated percentage of inflammatory monocytes, $\mathrm{F} 4 / 80^{+}$ macrophages, and $\mathrm{Ly}-6 \mathrm{G}^{+}$neutrophils (Figure 6). There was no increase in the percentage of CD19 ${ }^{+}$B-cells, CD4 T-cells, CD8 T-cells, Siglec- $\mathrm{F}^{+}$eosinophils or Ly-6C $\mathrm{C}^{\mathrm{lo}}$ resident monocytes (data not shown). This data are consistent with other investigations of hind limb ischemia and suggests that during revascularization 12/15-LOX activity may be derived from monocytes and macrophages. 
Isolated inflammatory monocytes are capable of producing RvD2-We next sought direct evidence of RvD2 production by inflammatory monocytes after confirming their infiltration during revascularization. While previous reports have demonstrated the expression of $12 / 15$-LOX in both monocytes and macrophages ${ }^{138}$, we chose to seek direct evidence for RvD2-synthesis by inflammatory monocytes because it is possible to isolate a pure population of these cells from the splenic reservoir. Using magnetic bead negative selection separation, we depleted all other leukocyte populations from isolated mouse spleens to collect only inflammatory monocytes (Figure 7B). Then, we incubated these monocytes with DHA substrate and simultaneously activated the cells with A23187 calcium ionophore for $30 \mathrm{~min}$. DHA-derived products were then extracted and identified by LC-MS/MS. We found that RvD2, RvD1, 14-HDHA, 17-HDHA, and 10,17-diHDHA, were all biosynthesized in large quantities (Figure 7C). To confirm that these lipids were produced by 12/15-LOX-metabolized precursors, we isolated the same population of inflammatory monocytes from mice missing the 12/15-LOX gene and repeated our in vitro stimulation and LC-MS/MS analysis. Our results showed that the RvD2, RvD1, 14-HDHA, 17HDHA, and 10,17-diHDHA production was either completely abolished or dramatically decreased. Collectively, these results indicate that inflammatory monocytes produce RvD2 by 12/15-LOX. 
Figure 5: 12/15-lipoxygenase biosynthesis products during revascularization. (A) 12/15lipoxygenase and 5-lipoxgenase oxygenate PUFAs at different carbon positions to yield different metabolites. (B) PUFA metabolites extracted from the hamstring muscles of ischemic limbs three days after occlusion. LC-MS/MS quantitated amounts were normalized to the weight of the harvested muscle. $n=5$ 
Figure 5

A.

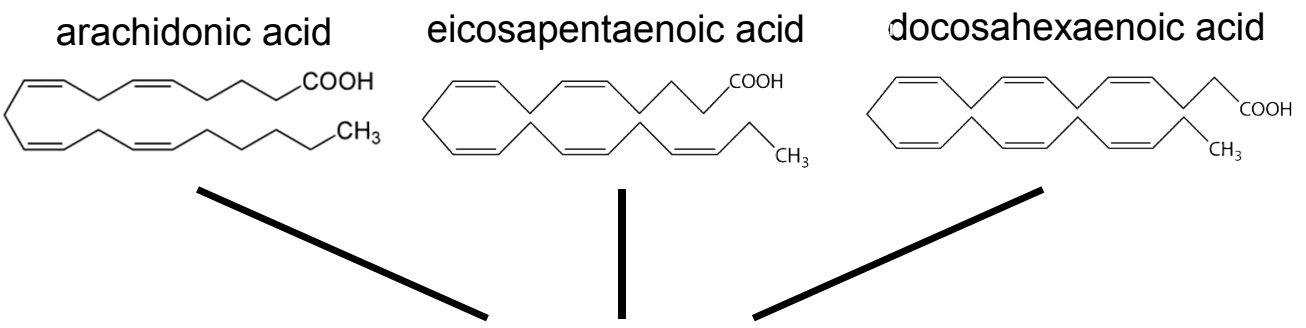

12/15-lipoxygenase

5-lipoxygenase

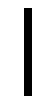

12-HEPE
12-HETE
15-HEPE
14-HDHA 17-HDHA
LTB4 5-HETE

4-HDHA

B.

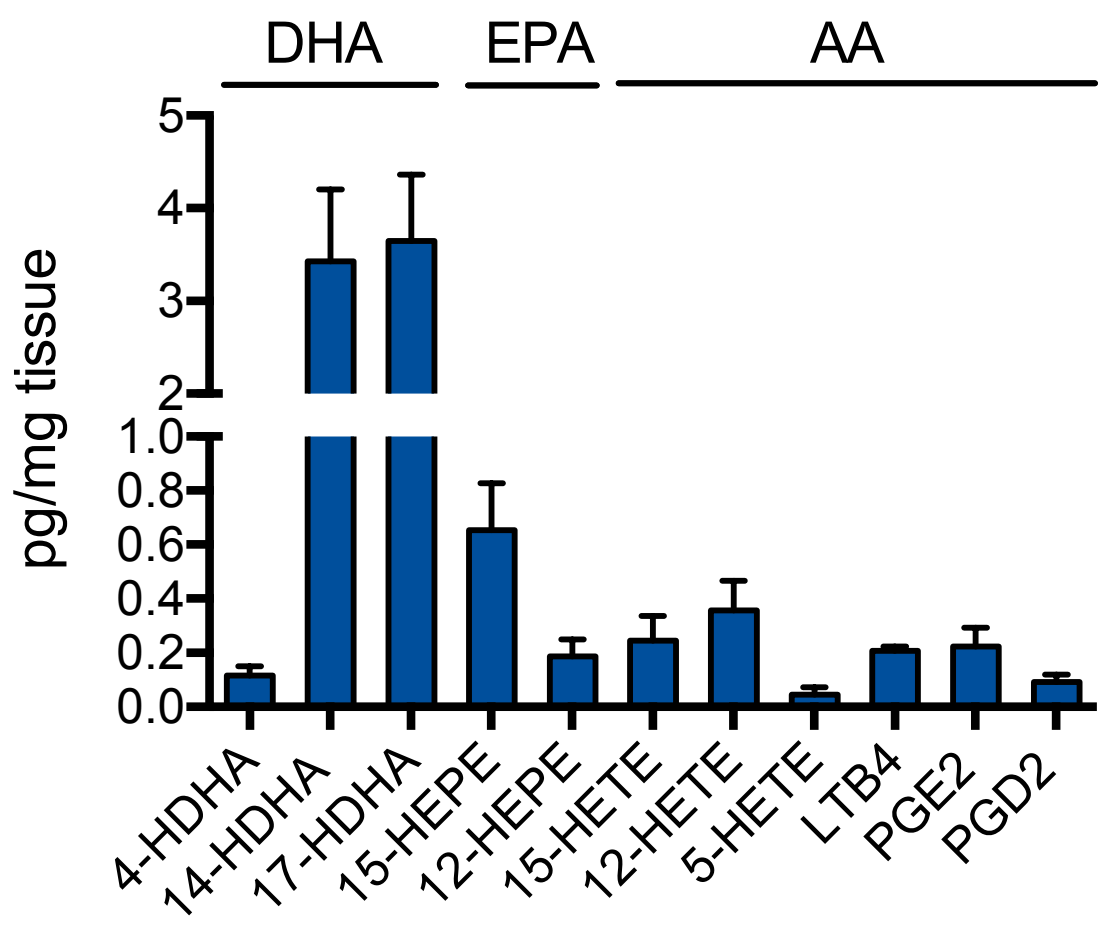


Figure 6: Leukocyte populations during revascularization. (A) Leukocyte population changes in the hamstring muscles during revascularization three days after induction of hind limb ischemia. The two axes used to gate populations are labelled on the $\mathrm{Y}$-axis. $\mathrm{Y}$-values are expressed as the percent of a population gate from the total count population. $\mathrm{n}=5$ per group, * indicates $p<0.05$. (B) Representative images of flow cytometry gates. 
Figure 6

A.
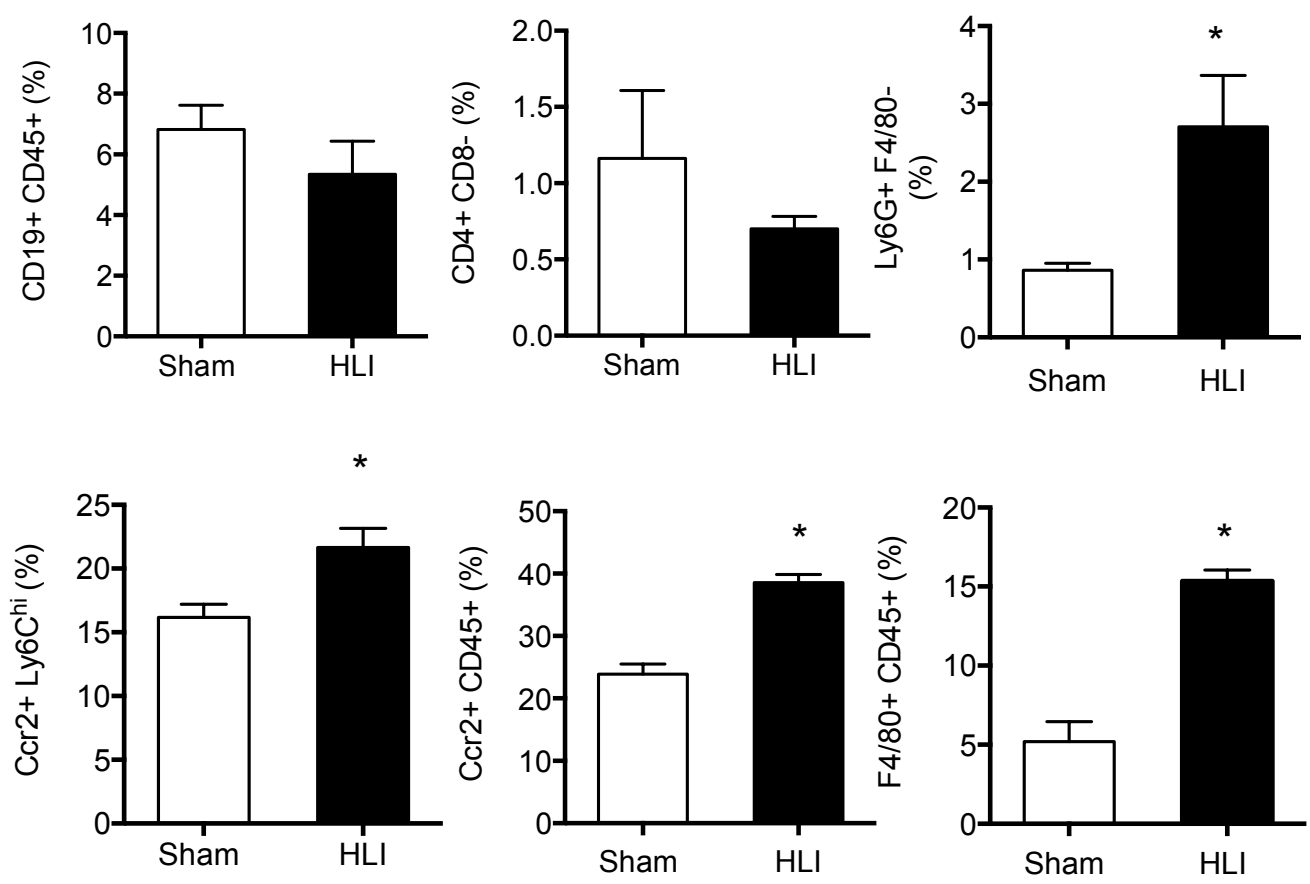

B.
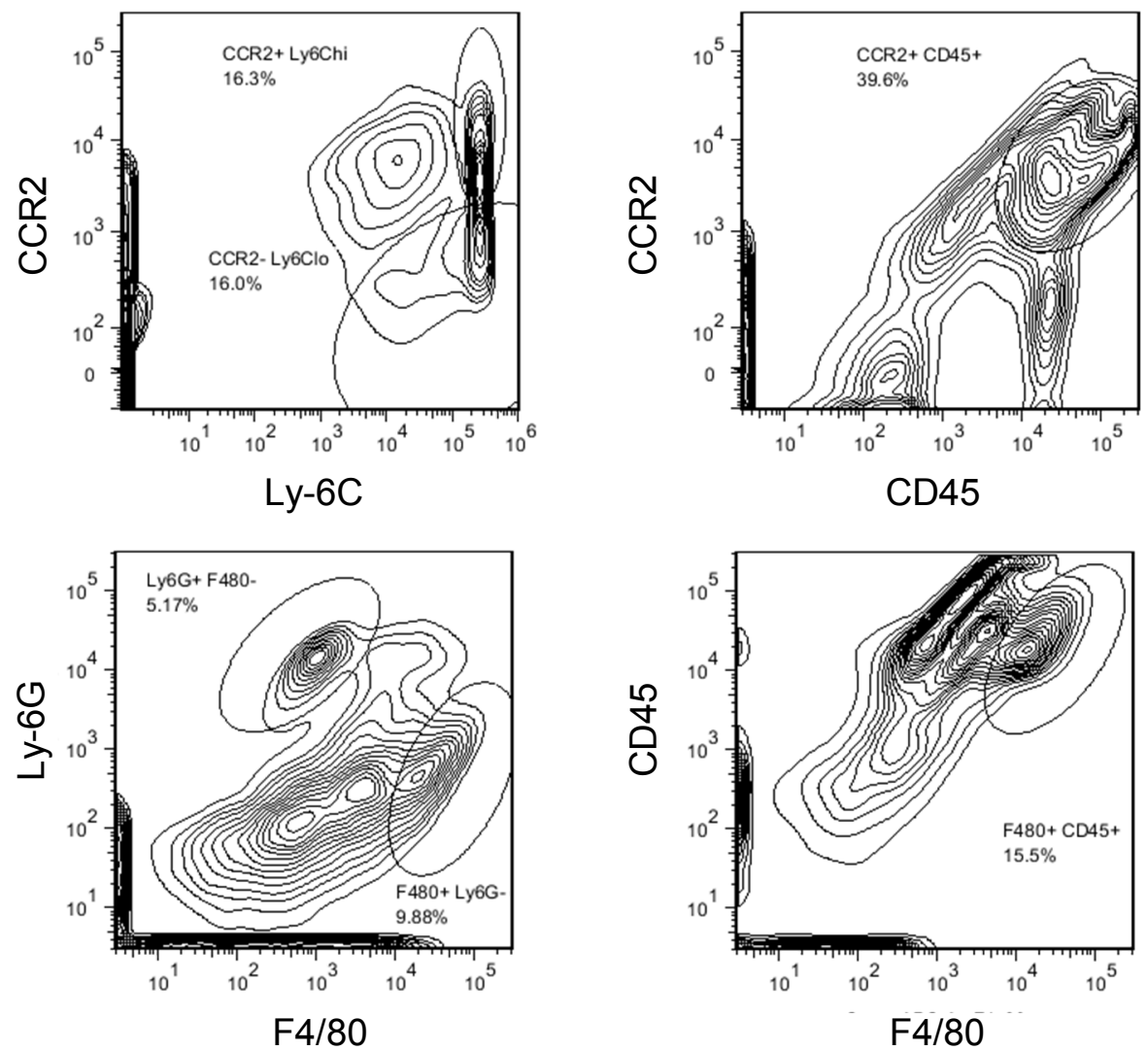
Figure 7: Ly- $6 C^{\text {hi }}, C C R 2^{+}$inflammatory monocytes produce RvD2. (A) Biosynthetic scheme of RvD1 and RvD2 from DHA substrate and 17-HpDHA intermediate by 12/15-LOX and 5-LOX enzymes. (B) Representative images of mouse spleen purification by magnetic bead negative selection separation to yield highly enriched population of Ly-6Chi, CCR2+ inflammatory monocytes. (C) Production of 12/15-LOX metabolites by splenic inflammatory monocytes from wildtype or 12/15-LOX KO mice. $n=3$ per group 
Figure 7

A.

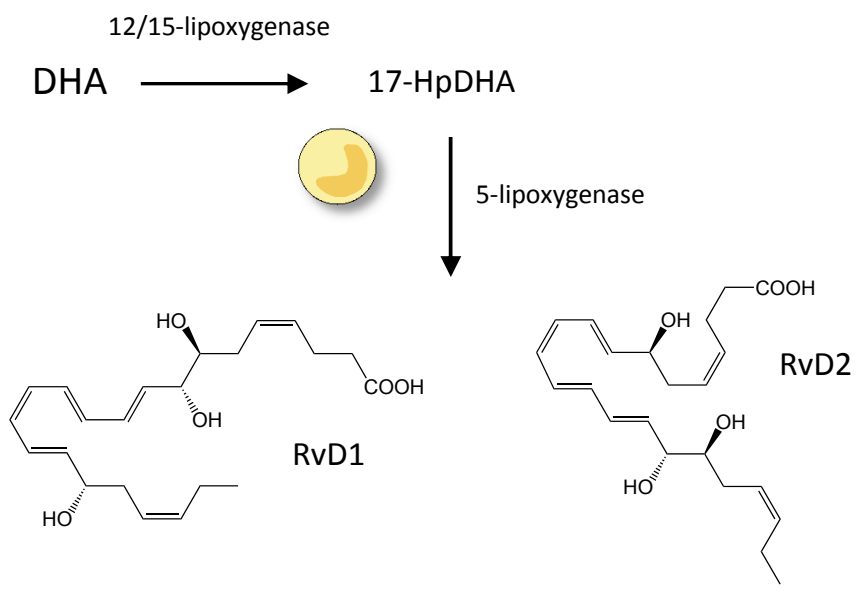

B.
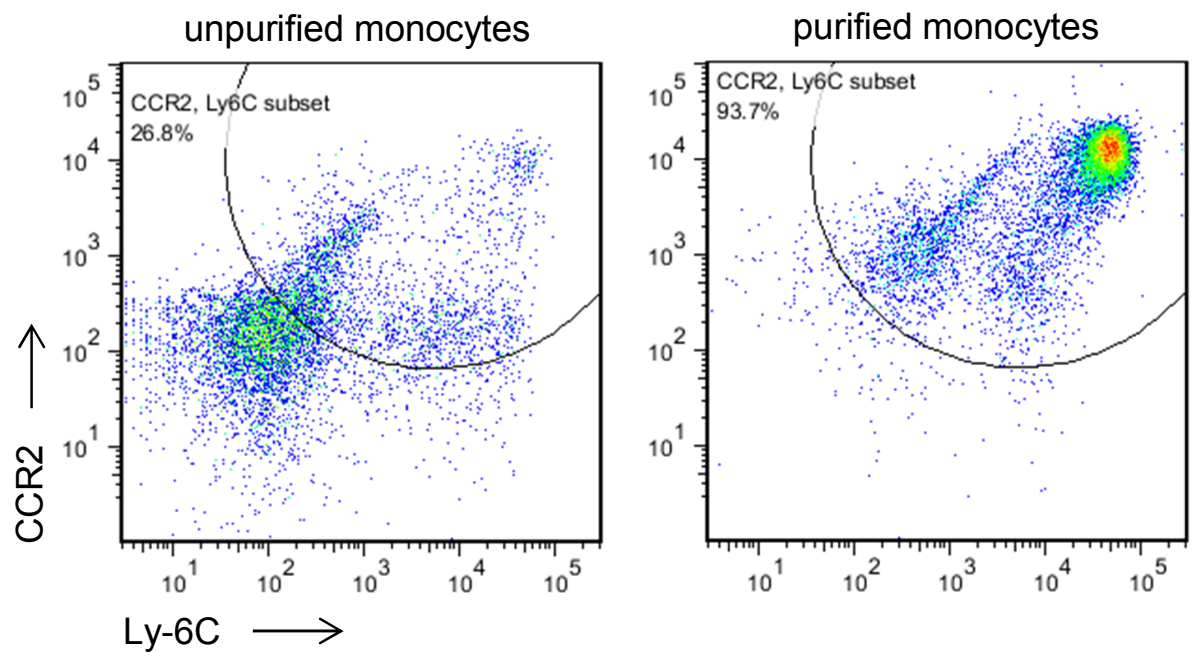

C.

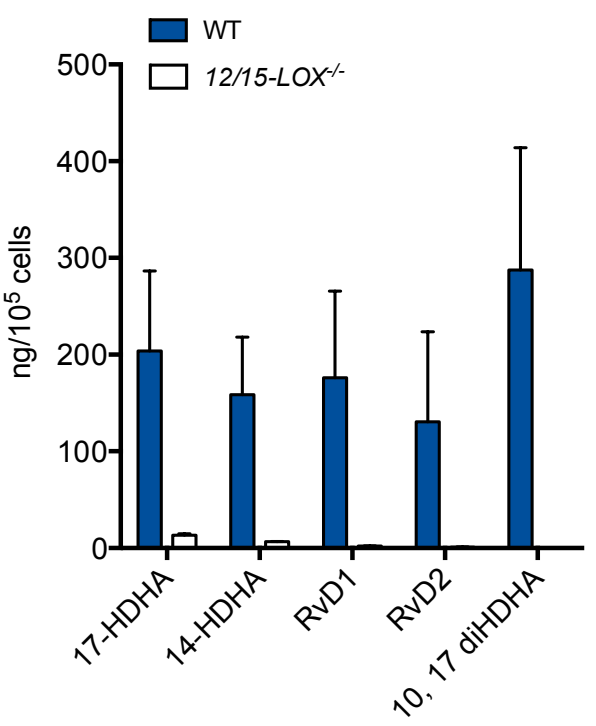




\section{Discussion}

The major finding of this study is that revascularization-elicited $L y-6 C^{\text {hi }}, C C R 2^{+}$ inflammatory monocytes express $12 / 15$-LOX and have the capacity to synthesize RvD2. We found the presence of 12/15-LOX and 5-LOX biosynthetic activity in mouse hind limbs three days after induction of ischemia, and an increase in inflammatory monocytes in the tissue at this same time. We then isolated this inflammatory monocyte population and stimulated them in vitro to demonstrate direct RvD2 production. Finally, we authenticated the 12/15-LOX origin of this RvD2 by ablating 12/15-LOX, which obliterated RvD2 synthesis. Overall, these results support the notion that infiltrating monocytes generate pro-resolving lipid mediators during post-ischemic revascularization.

Our 12/15-LOX and monocyte results are consistent with the conclusions of other investigations. Other studies show that $12 / 15$-LOX is important in the revascularization process ${ }^{92}$, and that the enzyme is expressed in monocytes and macrophages ${ }^{139}$. Previous studies suggest that $12 / 15$-lipoxygenase is important during revascularization because ablation of this enzyme hinders the ability of mice to recover blood perfusion in the hind limb ischemia model. However, this observation does not correspond directly to enzyme activity as the investigators did not measure 12/15-LOX enzyme products in vivo during revascularization. Thus we began our inquiry by examining 12/15-LOX activity during post-ischemia revascularization by identifying known 12/15-LOX lipid metabolites directly in mouse limbs. Because 12/15-LOX can be expressed in many different cell types, it was important that we attribute our observed 12/15-LOX activity specifically to monocytes and macrophages. The expression of 12/15-LOX in different monocyte and macrophage populations has been corroborated by many studies. Most germane to our investigation is not just the presence of 12/15-LOX mRNA or protein in monocytes and macrophages, but a demonstration of their RvD2-generating capability. We addressed this 
question by in vitro stimulation of spleen-isolated inflammatory monocytes to obtain 12/15-LOX products, including RvD2. Then, we further validated this enzyme-cell specificity by ablating 12/15-LOX and demonstrating the absence of previously detected enzyme products. This allowed us to confirm that inflammatory monocytes can produce RvD2.

Several studies suggest that monocytes and macrophages are critical to the revascularization process, and that inflammatory macrophages increase at injury sites during the early phases of repair. Monocytes and macrophages are critical to the revascularization process because in several specific knockout models depletion of these cells or inhibition of their normal functions attenuates post-ischemic recovery ${ }^{140}$. For example, granulocyte-colony stimulating factor deficient mice, which cannot differentiate monoblasts into mature blood monocytes or tissue macrophages, have significantly impaired revascularization ability ${ }^{141}$. Depleting monocytes and macrophages by 5 -fluorouracil or a diphtheria-toxin-CD11b-gene suicide-triggering system also decreases perfusion recovery after ischemia ${ }^{142}$. Mice that cannot produce $C C L 2$, the potent monocyte and macrophage chemoattractant, also have revascularization deficits ${ }^{143}$. The increase in monocyte and macrophage percentages in day-three post-ischemic limbs thus corroborates previous findings. Moreover, our observation of increased inflammatory monocytes but unchanged resident monocytes ( $\left(\mathrm{Ly}-6 \mathrm{C}^{\mathrm{l}}, \mathrm{CCR} 2^{-}\right)$specifically mirrored reports that there are two populations of peripheral blood that are present during different time points of inflammation. Inflammatory monocytes are rapidly recruited to sites of injury within hours, while resident monocytes appear many days later ${ }^{45}$. There is also evidence that resident monocytes at inflammatory sites are not new infiltrates; they are simply phenotype-switched original inflammatory monocytes ${ }^{144}$. Nevertheless, the spleen is a reservoir of both monocyte phenotypes $^{45}$. We not only measured an increase of inflammatory monocytes (and no change in resident monocyte) in our digested limb samples, but also isolated inflammatory monocytes from 
mouse spleens and confirmed their specific Ly-6C hi $^{\text {, CCR2 }}{ }^{+}$phenotype.

We are cognizant of the fact that inflammatory monocytes represent the largest proportion of leukocytes digested from limb muscles (20\%, Figure $6 \mathrm{~A})$. All the reported leukocyte percentages were gated on the total cell count; therefore the reported percentages are on an absolute scale. Additionally, inflammatory monocytes were not the only cell type increased as there was also an increase in the percentages of $\mathrm{F} 4 / 80^{+}$macrophages and $\mathrm{Ly}-6 \mathrm{G}^{+}$neutrophils. Nevertheless, we did not isolate and stimulate macrophages because F4/80 is a pan-macrophage surface marker that does not differentiate among the various macrophage phenotypes. Thus we were not confident in our ability to isolate a population of macrophages for in vitro stimulation that directly corresponded to cells that were increased during revascularization. Additionally, we did not isolate and stimulate neutrophils because we counted very few of them in digested muscle limbs and others studies have reported that although neutrophils express $12 / 15$-LOX, the enzyme has little activity until the cell is robustly stimulated ${ }^{145}$. Since neutrophils can have many states of differentiation and activation once they are mobilized from the bone marrow, we also did not isolate them for stimulation ${ }^{11}$.

There are other cell types that are increased during the revascularization process that we did not enumerate. Previous work has shown that B-cells and T-cells can also influence the revascularization process but again we did not observe any changes ${ }^{146}$. Various progenitor cells, fibroblasts, and vascular smooth muscle cells can all increase in post-ischemia limb tissue; however, we did not measure them ${ }^{147,148}$. The main reason is we only examined a single early time point during the revascularization process. Lymphocytes and mesenchymal cells appear predominantly later when the tissue is regenerating. Since our initial observations at day three post-occlusion were consistent with other reports, and the 12/15-LOX activity in these other cell types is not well documented, we did not pursue a comprehensive limb revascularization 
timecourse. In the future, a more comprehensive timecourse study of $12 / 15$-LOX activity and cell infiltration after hind limb ischemia would be needed to account fully for the various cell types than can produce RvD2 in post-ischemic tissue.

The appearance of EPA and DHA products in ischemic limb samples is consistent with observations that systemically available unesterified EPA and DHA can be shuttled to active sites of inflammation to act as a precursor of lipid mediators ${ }^{149}$. While humans cannot desaturate EPA and DHA natively and must derive it from their diet, EPA comprises $0.5-2.8 \%$ of total body fatty acids and DHA comprises approximately $0.3-5.0 \%{ }^{149}$. Mice do not possess PUFA desaturases either, but their diet contains EPA and DHA ${ }^{133}$. We also found metabolites of EPA and DHA in our pulverized ischemic limb samples. There were two noteworthy results in our data. One, tissue levels of 14-HDHA and 17-HDHA were significantly higher than EPA and AA 12/15-LOX products. Two, we were not able to detect resolvins in our limb samples. The availability of DHA at the revascularization site could be higher than EPA and $A A$, because there are many different phospholipase $\mathrm{A} 2 \mathrm{~s}$ and other classes of phospholipases, the actual liberation of DHA, EPA and AA free esters can be subject to complex regulatory mechanisms ${ }^{131}$. In specific contexts, there can be a preferential liberation of one PUFA over another. Secondly, our failure to detect RvD2 directly in post-ischemia limb tissue must be addressed. Normal murine resolution-phase peritoneal exudates contain only nanograms of RvD2 and supplementation of free DHA to the resolution site to drive substrate flux through the RvD2 biosynthetic pathway can boost synthesis, but the amount in muscle tissue is either less concentrated or harder to extract ${ }^{55}$. Therefore, a detectionlimit problem may have plagued our analysis of ischemic limb. In addition, like many other bioactive lipid mediators, RvD2 can be rapidly inactivated by dehydrogenases into degradation products that were not identified by our current protocol. Furthermore, we only examined only one time period after the induction of limb ischemia. While the low levels of PGE2 and LTB4 in our 
samples suggest the nadir of post-ischemia inflammation may have already passed, the peak of the resolution, during which RvD2 is abundantly generated, may be yet to occur. Finally, our rationale for studying the role of $\mathrm{RvD2}$ in revascularization was based on the dual immunovascular effects of this mediator and not because it is most abundant during inflammation, resolution or revascularization. Therefore a more comprehensive assessment of the timecourse of lipid mediator metabolism during revascularization is needed to understand the dynamics of RvD2 biosynthesis and the generation of other more endogenously preferred lipid mediators.

Finally, a few questions about RvD2 biosynthesis remain unanswered. The enzymatic conversion of the 7,8-epoxide intermediate into either RvD1 or RvD2 is not well described ${ }^{55}$. Are there one or two enzymes, and how is the production of RvD1 versus RvD2 regulated? We have also focused on 12/15-LOX in this study, but 5-LOX is also important in the biosynthesis of RvD2 (the second step). From our limb samples, it appeared that 5-LOX products, including RvD2, were not generated as abundantly as 12/15-LOX products; . Whether this lack of production was the result of enzyme regulation or substrate diversion needs to be elucidated. The 5-LOX is the ratelimiting enzyme in leukotriene synthesis, and perhaps as a result it also limits RvD2 synthesis ${ }^{31}$. Once again, the time period at which we measured 5-LOX metabolites may not coincide with its maximal enzymatic activity.

In conclusion, we have documented the specific RvD2-generating 12/15-lipoxygenase activity in ischemic injury-recruited Ly-6Chi CCR2+ inflammatory monocytes in this study. However, we did not directly detect RvD2 in the injured tissue of mice subjected to hind-limb ischemia. In the future, we plan to improve our analysis by performing a comprehensive temporal documentation of the various cell types and lipid mediators present during revascularization. 


\section{CHAPTER IV}

\section{MECHANISMS OF REVASCULARIZATION ENHANCEMENT BY RESOLVIN D2}

\section{Introduction}

Arteriogenesis and angiogenesis are the two processes by which new vasculature is formed in adult animals ${ }^{150}$. Arteriogenesis is the development process of preexisting collateral arterioles into arteries to circumvent an occlusion in a large artery, whereas angiogenesis is the growth of new capillaries by sprouting and intussusception of preexisting capillaries to perfuse areas of tissue hypoxia. Both processes are critical to revascularization and the restoration of blood perfusion in the ischemic tissue. Timely and robust revascularization is essential to stem tissue damage, such as in cases of myocardial infarction, ischemic stroke and critical limb ischemia. In our previous studies, we have demonstrated that the pro-resolving lipid mediator Resolvin D2 (RvD2) can enhance revascularization in a mouse model of critical limb ischemia. RvD2-treated animals have increased vascular volume in their ischemic limb and display reduced histologic signs of muscle damage. In addition, several other investigators have defined a clear role for monocytes and macrophages in the normal revascularization process, and they have demonstrated that transfusion of additional monocytes and macrophages can enhance revascularization ${ }^{72}$. We have provided evidence that $\mathrm{Ly}_{6 \mathrm{C}^{\text {hi }}} \mathrm{CCR} 2^{+}$inflammatory monocytes, which are highly present during limb revascularization, have the endogenous ability to synthesize RvD2, but the mechanism by which RvD2 enhances revascularization remains unclear. Previous work has shown that revascularization is mediated primarily by three cell types: monocytes and macrophages, vascular smooth muscle cells, and endothelial cells ${ }^{73}$. RvD2 could potentially affect 
all these cell types during revascularization.

Monocytes and macrophages can synthesize RvD2, and they can be stimulated by RvD2 in a paracrine or autocrine manner ${ }^{137}$. RvD2 is synthesized from docosahexaenoic acid (DHA) by 12/15-lipoxygenase and 5-lipoxygenase ${ }^{55}$. Monocytes and macrophages express both enzymes, and our studies from our laboratories and those of others have demonstrated that in vitro, these cells can synthesize RvD2. Once synthesized, RvD2 can in turn stimulate monocytes and macrophages. In a mouse zymosan-induced peritonitis model, intravenous injection of 100ng of RvD2 prior to zymosan-stimulus resulted in greater monocyte infiltration into the peritoneum. Isolated mouse peritoneal macrophages also are stimulated by RvD2 to ingest FITC-labelled zymosan particles in an in vitro phagocytosis assay. Thus RvD2 can be biosynthesized by monocytes and macrophages, and it can also directly stimulate them. Nevertheless, other cell types can be affected by RvD2 as well.

Vascular smooth muscle cells (VSMC) are another target of RvD2. A recent investigation provides detailed insights into its stimulatory actions in $\mathrm{VSMC}^{151}$. In vitro, RvD2 attenuates the proliferation of VSMC in basal culture conditions, and in migration chambers RvD2 decreased VSMC chemotaxis toward a PDGF gradient. RvD2 also decreases TNF $\alpha$-stimulated expression of VCAM-1, ICAM-1, IL-1 $\beta$, IL-6, and CCL2 mRNA in VSMC. In vivo, RvD2 decreased rates of cell proliferation, as measured by Ki-67 staining, in the vessel wall of balloon catheter-injured rabbit femoral arteries. In histologic sections of these arteries, neointimal hyperplasia was found to be decreased. These data are consistent with the in vitro results showing the anti-proliferative effects of RvD2. In addition, RvD2 can also decrease ROS production as indexed by dihydroethidium staining, leukocyte infiltration, and inflammatory cytokine production (TNFa, IL-1, CCL2) in femoral arteries. Collectively, these data provide evidence, which suggests that RvD2 elicits an inhibitory and anti-proliferative effect on VSMC. 
RvD2 can also stimulate endothelial cells ${ }^{55}$. In mouse ears, topical application of $100 \mathrm{pg}$ of RvD2 dilates the ear microvasculature as quantitated by increased FITC-albumin signal. Interestingly, application of a nitric oxide inhibitor (L-NAME) abolished this dilatory effect, suggesting a potential nitric oxide-dependent mechanism. In the mouse cremaster microcirculation model, RvD2 administration was found to decrease platelet activating factorstimulated neutrophil adhesion to the endothelium. This observationhas been replicated in vitro in a flow chamber, and it was found that RvD2 decreased PAF-stimulated capture and adhesion of neutrophils by human umbilical vein endothelial cells (HUVEC). Additionally, RvD2 directly stimulates HUVECs to produce more prostacyclin and nitric oxide, two highly potent vasoactive mediators. In a separate investigation, the vasoactivity of RvD2 was found to prevent secondary thrombosis of deep dermal vascular network after burn injury to aid in the preservation of microvasculature ${ }^{152}$. Together, this body of evidence indicates that in addition to its established immunomodulatory actions, RvD2 has potent vascular effects.

RvD2 can enhance revascularization and affect the three predominant cell types involved in the revascularization process, but significant mechanistic questions remain unanswered. Specifically, it remains to be clarified whether RvD2 can directly stimulate arteriogenic and angiogenic gene expression in these cells, or whether RvD2 affects endothelial proliferation and migration. Results of our present study provide evidence that RvD2 does not stimulate arteriogenic, angiogenic, or inflammatory gene expression in isolated macrophages or endothelial cells. Our results also show that RvD2 does not stimulate angiogenesis in an in vivo Matrigel plug assay, and it does not enhance endothelial cell proliferation. However, we found that RvD2 stimulates endothelial migration in a Rac-1 dependent manner. As endothelial migration is important for mechanotaxis during the changes in shear stress experienced after arterial occlusion and during arteriogenesis, this evidence is consistent with the view that during revascularization 
RvD2 enhances arteriogenesis, but not angiogenesis by stimulating endothelial migration.

\section{Experimental procedures}

Multiplex ELISA analysis of mouse plasma-Mouse peripheral blood was collected by cardiac puncture, anti-coagulated with $16 \mu \mathrm{L}$ of $200 \mathrm{mM}$ EDTA, and centrifuged at $2000 \mathrm{xg}$ for $20 \mathrm{~min}$. The plasma supernatant was collected and frozen at $-80^{\circ} \mathrm{C}$. Multiplex ELISA analyses were then performed by Quansys Biosciences.

\section{Realtime reverse transcriptase polymerase chain reaction- Mouse hamstring and} gastrocnemius were harvested, snap-frozen in liquid nitrogen, and powdered with a tissue pulverizer. The tissue powder was incubated and vortexed in TRIzol (Life Technologies) for 5 min. Afterwards, samples were incubated with chloroform for $3 \mathrm{~min}$ and then vortexed at $12,000 \times \mathrm{g}$ for 15 minat $4^{\circ} \mathrm{C}$. The upper aqueous phase was then transferred to a new tube and mixed with 1 volume of $70 \%$ ethanol. Isolated peritoneal macrophages and Mile Sven 1 (MS1) endothelial cells were scraped with a cell scraper and lysed with Buffer RLT (Qiagen). Total RNA solutions from either mouse tissue, or cell lysate in Buffer RLT, were then purified with an RNeasy kit (Qiagen). RNA quality was checked by $260 / 280$ UV absorbance ratio with a Nanodrop (Thermo). cDNA was prepared by polymerase chain reaction using avian myeloblastosis virus reverse transcriptase and oligoDT primers. The PCR cycling parameters were one cycle of $95^{\circ} \mathrm{C}$ for $10 \mathrm{~min}$, then 40 cycles of $15 \mathrm{~s}$ at $95^{\circ} \mathrm{C}$ and $60 \mathrm{~s}$ at $60^{\circ} \mathrm{C}$.

The cDNA was then analyzed by real-time PCR by the SYBR green and $\triangle \triangle C$ t method to quantitate the reverse-transcribed messenger RNA. The CDNA samples were mixed in duplicate with specific RT-PCR forward and reverse primers (SA Bioscences or IDT DNA) and PerfeCTa SYBR Green FastMix (Quanta Biosciences). The hypoxanthine-guanine phosphoribosyltransferase 
(HPRT) housekeeping gene was used as internal quantitation control. Samples were then measured with a Prism 7900 HT (Applied Biosystems). The realtime cycling parameters were one 5 min cycle at $95^{\circ} \mathrm{C}$, then 45 cycles of $10 \mathrm{~s}$ at $95^{\circ} \mathrm{C}, 20 \mathrm{~s}$ at $60^{\circ} \mathrm{C}$, and $25 \mathrm{~s}$ at $72^{\circ} \mathrm{C}$. SYBR green fluorescence from amplified cDNA was recorded at the end of each cycle and the number of cycles at which a sample crossed the threshold value was noted. Quantitation of mRNA expression was then performed using the $2^{-\Delta \Delta \mathrm{Ct}}$ mathematical model, where $\Delta \Delta \mathrm{Ct}=\Delta \mathrm{Ct}_{\text {gene of }}$ interest $-\Delta \mathrm{Ct}_{\mathrm{hprt}}$

Matrigel plug assay-Matrigel is an extracellular matrix mixture secreted by Engelbreth-HolmSwarm mouse sarcoma cells ${ }^{153}$. A $500 \mu \mathrm{L}$ aliquot of Matrigel (Cultrex HC, Trevigen) was thawed on ice and mixed with either no additives, 500ng FGF2 + 50U heparin, 100ng RvD1, or 100ng RvD2. The mixture was then loaded into syringes with attached 25 gauge needle. The mouse was then anesthetized with isoflurane and denuded with depilatory cream on the right lateral abdomen. The Matrigel mixture was then injected subcutaneously under the skin and allowed to solidify into plugs for $20 \mathrm{~min}$. The mouse was then allowed to awaken and recover for seven days. On the seventh day, the mouse was euthanized and the Matrigel plug was excised. To release the infiltrated endothelial cells, the Matrigel plugs were digested with $5 \mathrm{mg} / \mathrm{mL}$ of Type I and II collagenase in $1 \mathrm{~mL}$ of Hank's Balanced Salt Solution for 1 hour at $37^{\circ} \mathrm{C}$. Afterward, digested plugs were strained with a $40 \mu \mathrm{M}$ cell strainer and rinsed with PBS-BSA. The cell solution was then stained with Fc-block (anti-CD16/32) and FITC anti-CD45, PE anti-CD31, PE/Cy7 anti-CD105, and APC anti-CD144. After a final rinse, cells were analyzed on an Accuri flow cytometer.

Scratch migration assay-MS1 cells were seeded at one millions cells per $6 \mathrm{~cm}$ cell culture well the night prior to experimentation in 3mL of Dulbecco's Modified Eagle Medium (DMEM). Using a 
$200 \mu \mathrm{L}$ pipette tip, two vertical scratches were made across the culture dish. The scratch was then imaged and the medium was replaced with fresh media plus stimuli. Eighteen hours later, the plates were imaged again and discarded. The $0 \mathrm{~h}$ and 18 himages were then processed by Photoshop (Adobe) with vertical lines demarcating migration of cells into the scratch site, and then analyzed for migrated width using Image J (NIH).

MTT \& EdU proliferation assay-MS1 cells were seeded at 10,000 cells per 96 -well well in $100 \mu \mathrm{L}$ of $1 \%$ serum DMEM overnight. The next morning, cells were given new media with various stimuli and incubated at $37^{\circ} \mathrm{C}$ for $48 \mathrm{~h}$. Cell proliferation was measured using an MTT assay kit (Invitrogen) or EdU kit (Molecular Probes) and quantitated on a microplate reader (Biotek).

Rac1 pulldown assay-MS1 cells were cultured on $150 \mathrm{~mm}$ plates in $30 \mathrm{~mL}$ of DMEM with $10 \%$ FBS until half confluent. Culture media was then changed to 1\% serum DMEM for 18 hours. Cells were then stimulated with 1nM RvD2 or unstimulated and VEGF controls for $30 \mathrm{~m}$. Plates were then rinsed with cold PBS to halt the reaction. Cells were then lysed and phosphorylated Rac1 was precipitated with a Rac1 activation kit from CellBiolabs.

Western blotting-Rac1 pulldown samples were separated by sodium dodecyl sulphate polyacrylamide gel electrophoresis and transferred to polyvinylidene difluoride (PVDF) membranes. The membrane was blocked overnight in tris-buffered saline with $1 \%$ tween 20 (TBST) and 5\% powdered milk. Precipitated Rac1 and total Rac1 was then probed with anti-Rac1 (Cell Signaling Technologies) and anti-rabbit-HRP in TBS-T with 1\% BSA. Membranes were then developed with HRP substrate (Millipore) and scanned with a Typhoon bioimager (GE Healthcare). Densitometry of immunoblot bands was performed in ImageJ. 


\section{Results}

\section{Leukocyte numbers in ischemic hamstring muscles are not significantly altered by RvD2}

treatment-In our previous study, we demonstrated that our hind limb ischemia model elicited an increase in certain myeloid cells and did not alter other leukocytes three days after occlusion. We then asked if RvD2 can alter any of these leukocyte populations. To address this question, we digested ischemic semitendinosus, semimembranosus and biceps femoris (hamstring) muscles, 3 days post-occlusion, and enumerated leukocytes as a percentage of total counts. We found that there was no statistically significant difference in neutrophil and macrophage percentages in vehicle versus RvD2-treated limbs (Figure 8A). RvD2-treatment also did not change the percentage of $\mathrm{Ly} \mathrm{C}^{\mathrm{hi}} \mathrm{CCR}^{+}$inflammatory monocytes (Figure 8B), Ly6C ${ }^{\text {lo }} \mathrm{CCR}^{-}$resident monocytes, B-cells and CD4 T-cells. Overall, RvD2 treatment during revascularization did not alter leukocyte populations in the ischemic muscle compared with vehicle treatment on day 3 after occlusion.

Plasma cytokine changes three days after HLI-The systemic plasma levels of cytokines can change after local ischemia. Therefore, we measured a panel of cytokines that are often increased during revascularization (Figure 9). Changes in fluid shear stress can stimulate endothelial cells to release CCL2. We did not detect a statistically significant increase in CCL2 after HLI. There was a strong upward trend however, suggesting simply a lack of statistical power. Regardless, there was no change in CCL2 with RvD2 treatment either. Consistent with other studies, RvD2-treated HLI mice possessed lower circulating TNF $\alpha$, a major inflammatory cytokine, compared with vehicletreated mice. There was a similar decrease in GM-CSF with RvD2 treatment, too. Conversely, IL-4 and IL-10 were increased in RvD2 mice versus vehicle mice. IL-10 was not detected in any of the 
control groups (sham treatments, and vehicle-HLI), and was present only in RvD2-treated mice. Collectively, these results indicate that RvD2 can alter systemic levels of important cytokines that mediate revascularization.

mRNA expression changes in ischemic hamstring muscles three days after HLI-Hind limb ischemia can also alter the expression of revascularization-mediating genes. To measure these genes, we isolated total RNA from vehicle or RvD2-treated ischemic hamstring muscles three days after surgery, and measured mRNA expression of a panel of arteriogenic and angiogenic genes. When normalized to vehicle-treated sham hamstrings, we did not observe statistically significant changes, by multiple comparisons (T-tests), in Vegfa, Vegfr2, Tnfa, Tie2, Vcam, Ccl2, Arg1, Il1b, Emr1, and Il6 (Figure 10A). However, although our sample size was small and lacked statistical power, there were some encouraging trends. Subsequent analysis comparing RvD2-treated samples only with vehicle-treated samples resulted in a statistically significant increase in Vegfa, Tie2, Arg1 (Figure 10B). The fold changes in the genes were modest however, and taken together the RT-PCR data suggest that RvD2 does not significantly affect gene transcription in ischemic limb during revascularization.

mRNA expression changes in RvD2-stimulated peritoneal macrophages-We also measured direct modulation of gene transcription in macrophages by RvD2. For this, we isolated peritoneal macrophages from wild-type mice and stimulated cells in vitro for $4 \mathrm{~h}$ with $0.1 \mathrm{nM}$ RvD2. We measured the mRNA of the three genes elevated in the muscle samples, Vegfa, Tie2 and Arg1, but did not find any changes (Figure 10B). There were also no changes in $C c l 2$, Tnfa, $\|l 1 b\| l 6,, \| l 10$, Mmp2, Pdgfa, Nos2, Cxcl12, Ptgs2, Alox5, or Alox 15. We did measure an increase in Mmp14 mRNA with RvD2 treatment. Mmp14 encodes membrane type 1 metalloproteinase (MT1-MMP), which is 
involved in the activation of other metalloproteinases during matrix remodeling. With the exception of Mmp14, RvD2 did not robustly stimulate gene transcription in macrophages.

RvD2 stimulates endothelial migration but not proliferation-Endothelial cells are critical participants in revascularization. They perform two essential activities, i.e., migration and proliferation $^{71}$. We thus examined if RvD2 affects either of these two activities. Using MS1 mouse microvascular endothelial cells, we performed in vitro scratch assays with different doses of RvD2 and found that the rate of scratch wound closure was enhanced (Figure 12A). We also performed cell proliferation assays employing 5-ethynyl-2'-deoxyuridine (EdU) incorporation, Hoescht nuclear dye or MTT tetrazole as markers of proliferation. In the EdU assay, RvD2 did not stimulate an increase in EdU incorporation. In the Hoescht and MTT assays, we did not find any RvD2stimulated increase in MS1 cell number (Figure 12B). These data suggest that RvD2 can enhance endothelial migration but not proliferation.

RvD2 does not stimulate angiogenic gene expression changes in endothelial cells-Because RvD2 can directly stimulate endothelial cells, we also asked if any genes were simultaneously transcribed. After stimulating MS1 cells for $4 \mathrm{~h}$, total RNA was isolated and mRNA was quantitated by RT-PCR. There was no difference in Vegf1, Tie2, Ccl2, and Mmp14 (Figure 13A). Thus we did not uncover any RvD2-stimulated gene transcription in endothelial cells.

RvD2 does not stimulate angiogenesis-The in vivo Matrigel plug assay is a simple but effective method to assay angiogenic effects of study compounds ${ }^{153}$. Many investigators harvest the Matrigel plug one week after implantation and perform histologic analysis. We chose instead to use high-throughput flow cytometer-detection of CD31 and CD105-labelled endothelial cells in 
collagenase-digested plugs. Matrigel plugs containing no compounds (negative control), basic fibroblast growth factor (bFGF, 500ng, positive control) or RvD2 (100ng) were implanted and harvested after 7 days. bFGF stimulated a robust infiltration of endothelial cells, giving the excised plug a red appearance and firm texture (Figure 13B). RvD2-infused plugs were no different from negative control plugs; they retained no red color, disintegrated during tissue excision, and contained few endothelial cells. The results of this assay suggest that RvD2 does not stimulate angiogenesis.

RvD2 stimulates migration through eNOS and Rac1-Endothelial migration can be stimulated by many pathways ${ }^{154}$. To gain further insights into which pathways RvD2 stimulates in endothelial cells, we performed scratch assays with inhibitors to two important mediators of migration: L-NGnitroarginine methyl ester to inhibit endothelial nitric oxide synthase (eNOS); NSC23766 to inhibit Rac1. Both compounds ablated the stimulatory effect of RvD2 on MS1 migration (Figure 14A). To provide further evidence of Rac1 pathway involvement, we used a Rac1 activation assay to measure RvD2-stimulated GTP binding to Rac1. The activation assay is a bead precipitation procedure using the PAK protein binding domain to capture only GTP-bound (ie. active) Rac1. Precipitated Rac1 is then normalized with total cellular Rac1 by immunoblot and quantitated by densitometry. RvD2 stimulation of MS1 cells increased the proportion of active GTP-bound Rac1 to total Rac1 (Figure 14B). Results from the scratch assay and activation assay suggest that RvD2 promotes endothelial migration by regulating Rac1 and eNOS. 
Figure 8: Leukocytes numbers in ischemic muscles are not altered by RvD2. Hamstring muscles from the ischemic limb of mice, treated with saline vehicle or RvD2 for $3 \mathrm{~d}$ after induction of HLI, were excised and digested by collagenases to liberate individual cells. Populations of leukocytes were identified by a panel of indicated surface markers and enumerated by flow cytometry. (A) Representative contour plots of neutrophil vs macrophage flow cytometry and quantitated graphs of all experiment groups. (B) Quantitation of other leukocyte populations in digested ischemic hamstring muscles. $n=5,{ }^{*} p<0.05$ 
Figure 8

A.
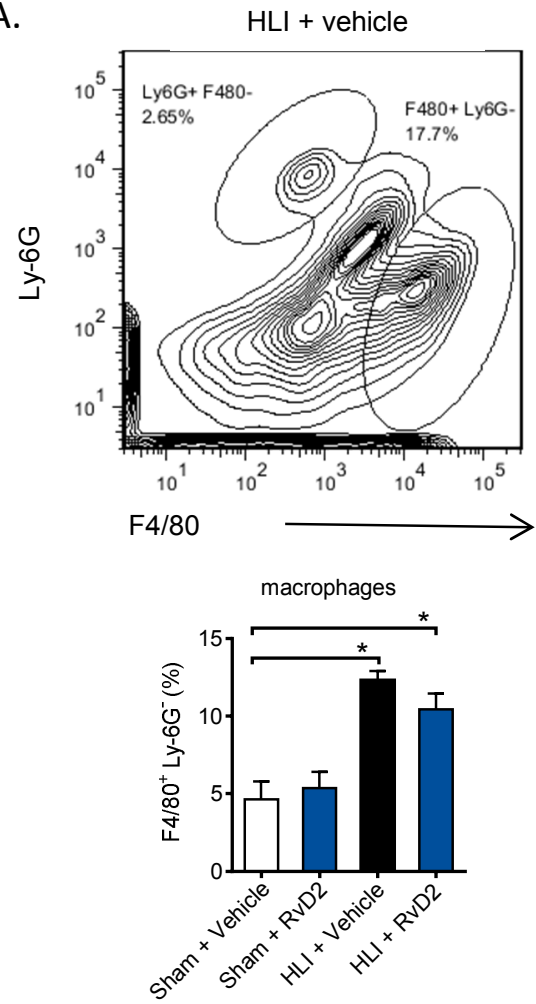

HLI + RvD2
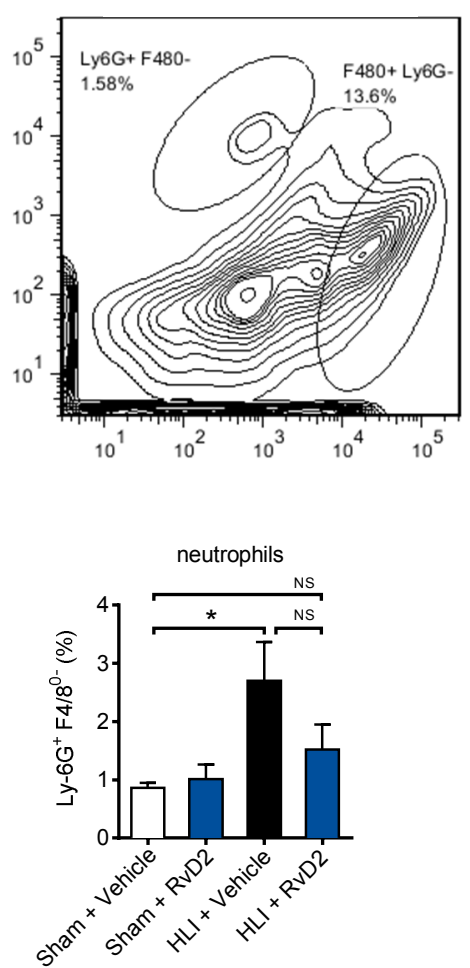

B.
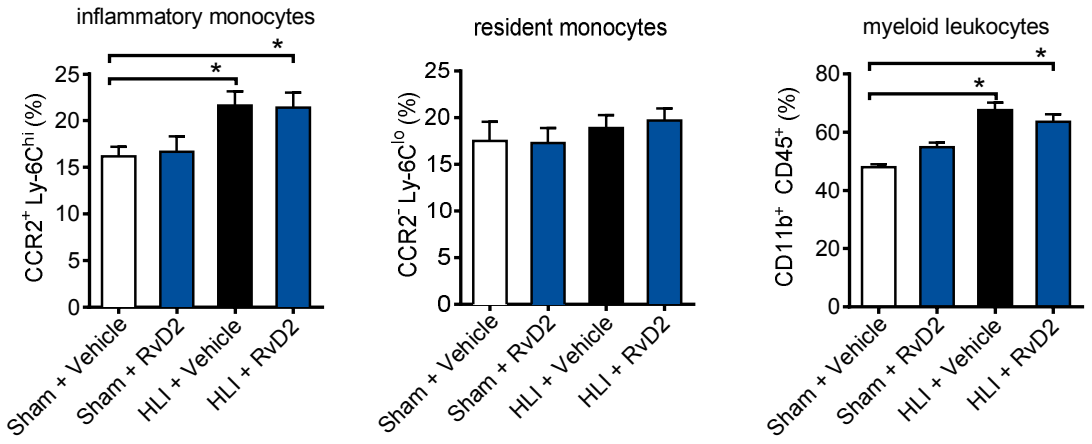

CD4 T-cells
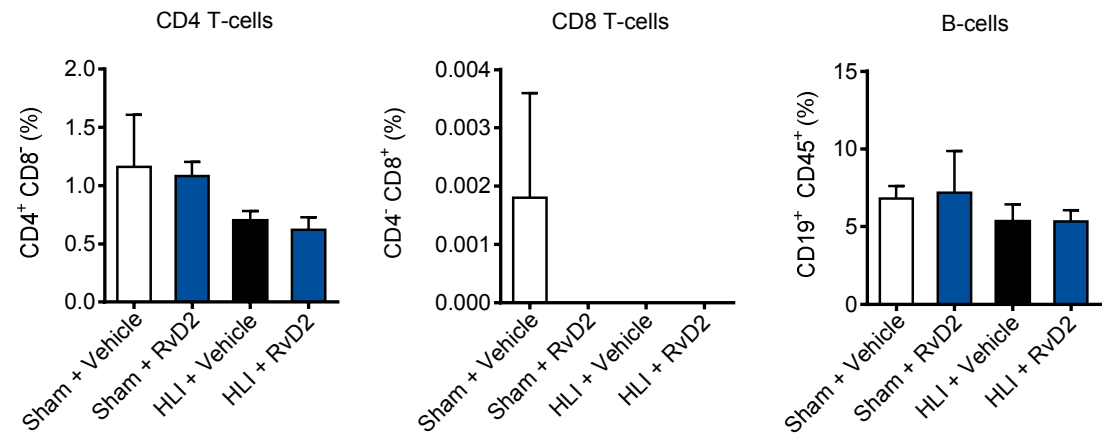
Figure 9: Plasma cytokine changes three days after HLI. TNF $\alpha$, IL-4, IL-10 and GM-CSF cytokine levels in peripheral blood of $3 \mathrm{~d}$ HLI mice. $n=5,{ }^{*} p<0.05$ 
Figure 9
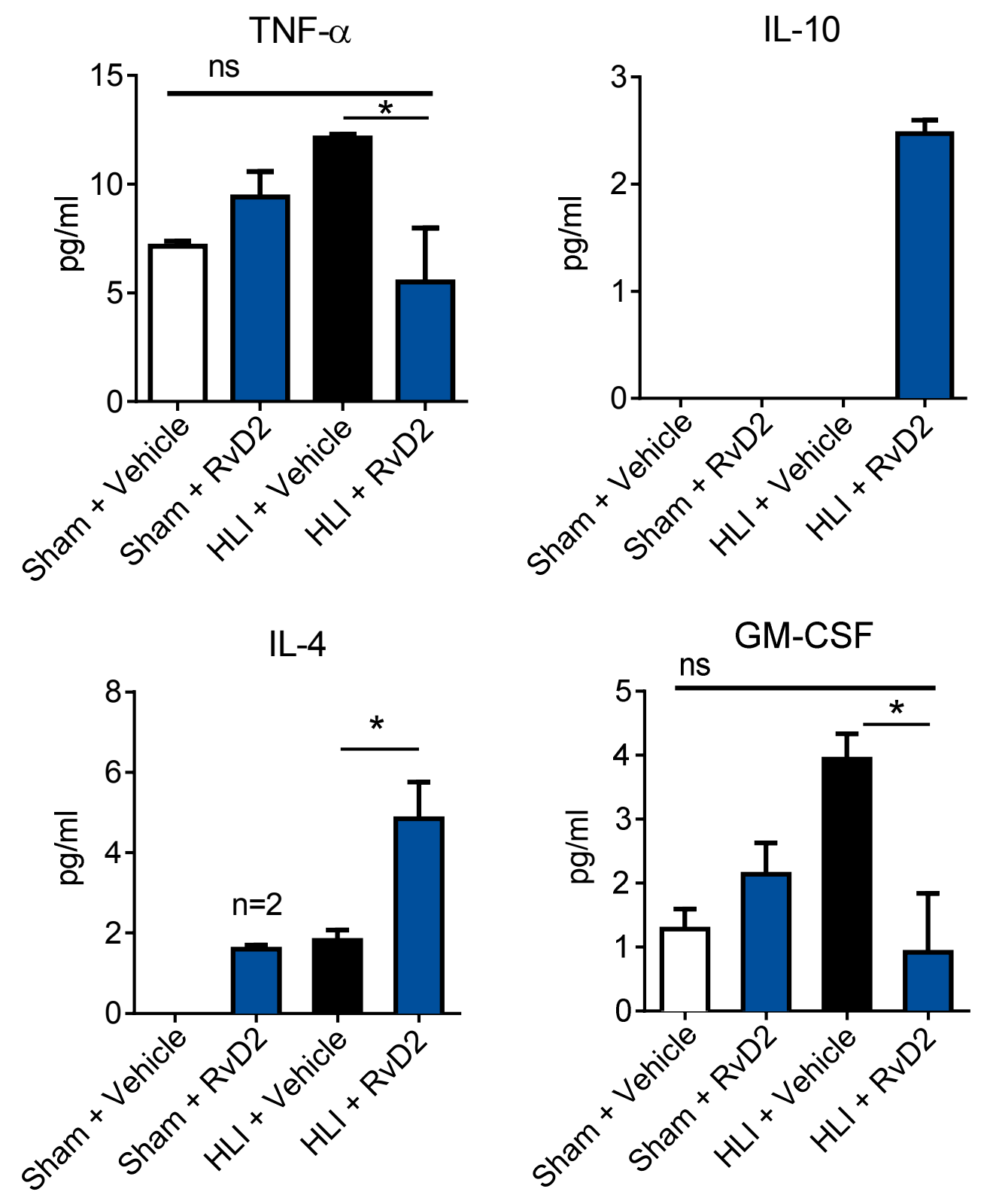
Figure 10: mRNA expression changes in hamstring muscles $3 \mathrm{~d}$ after HLI. (A) Quantitation of mRNA in $3 \mathrm{~d} H \mathrm{HLI}$ ischemic hamstring muscles. Relative expression was calculated by comparing to average Hprt expression in vehicle-treated sham surgery samples. (A) Same samples but with relative expression calculated by comparing to average Hprt expression in vehicle-treated HLI surgery samples. $n=5,{ }^{*} p<0.05$ 
Figure 10

A.

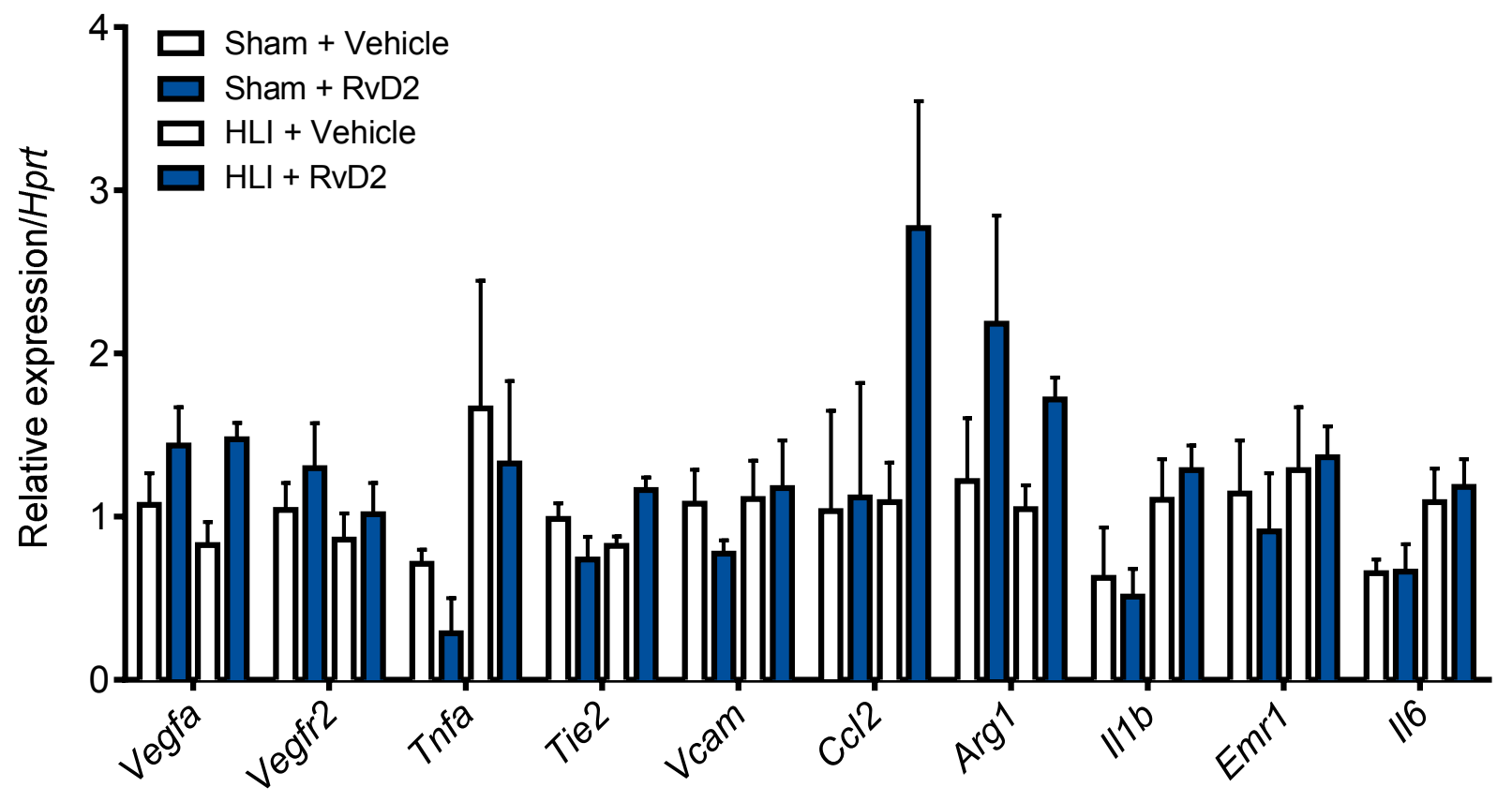

B.

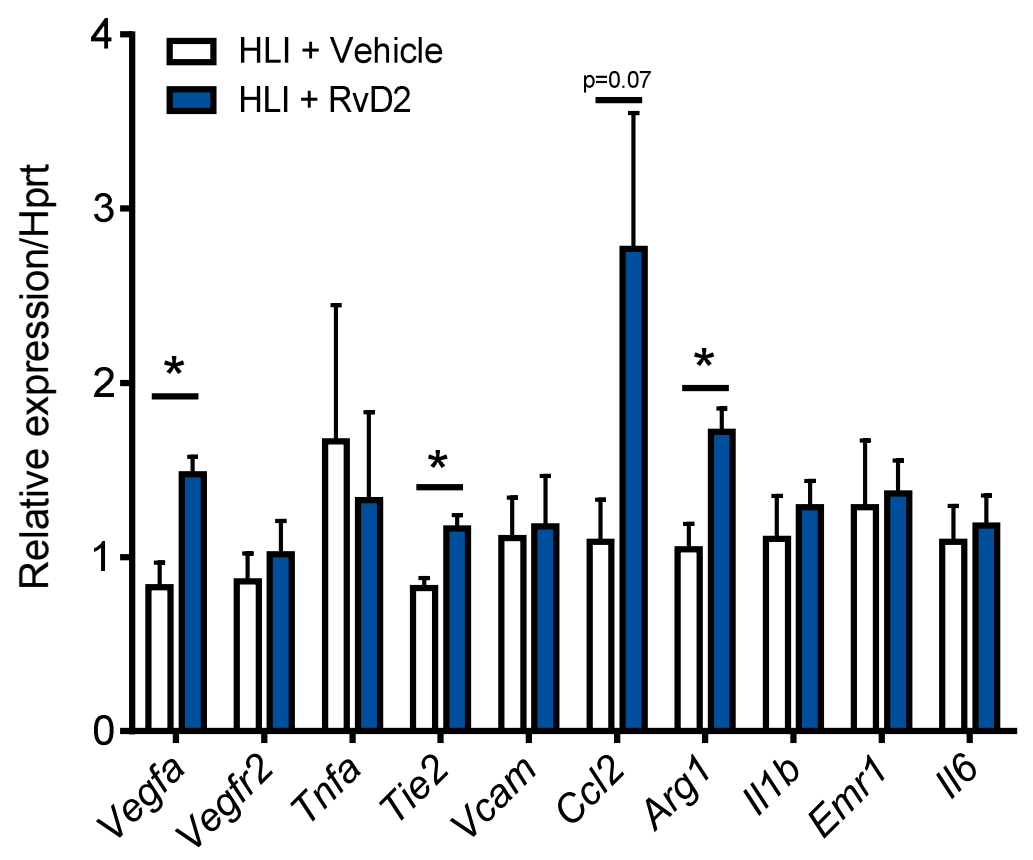


Figure 11: mRNA changes in RvD2-stimulated peritoneal macrophages. RT-PCR of isolated mouse peritoneal macrophages stimulated with $0.1 \mathrm{nM}$ RvD2 for 4 hours. $n=7-9,{ }^{*} p<0.05$ 
Figure 11
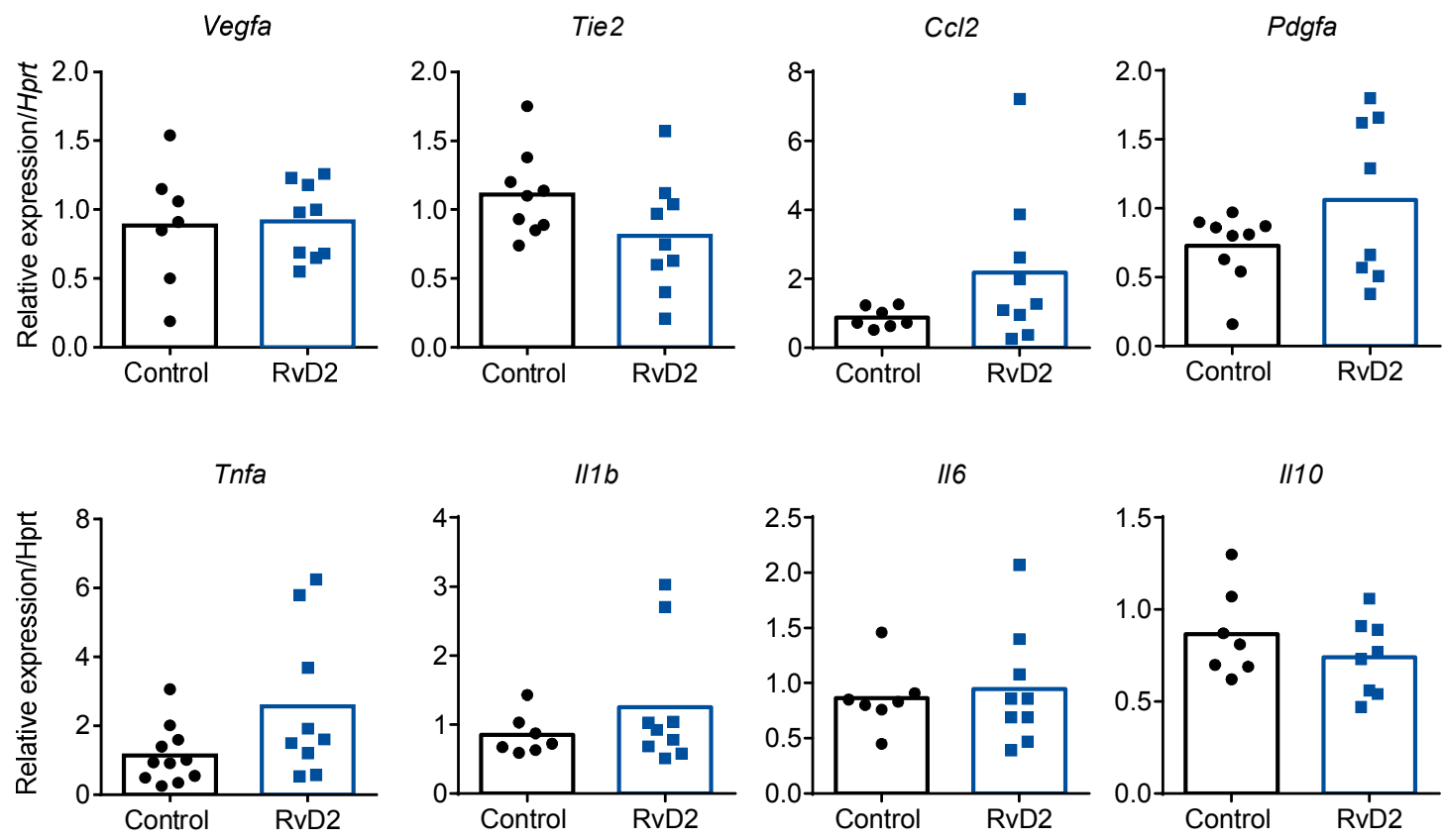

II1b

116

II10
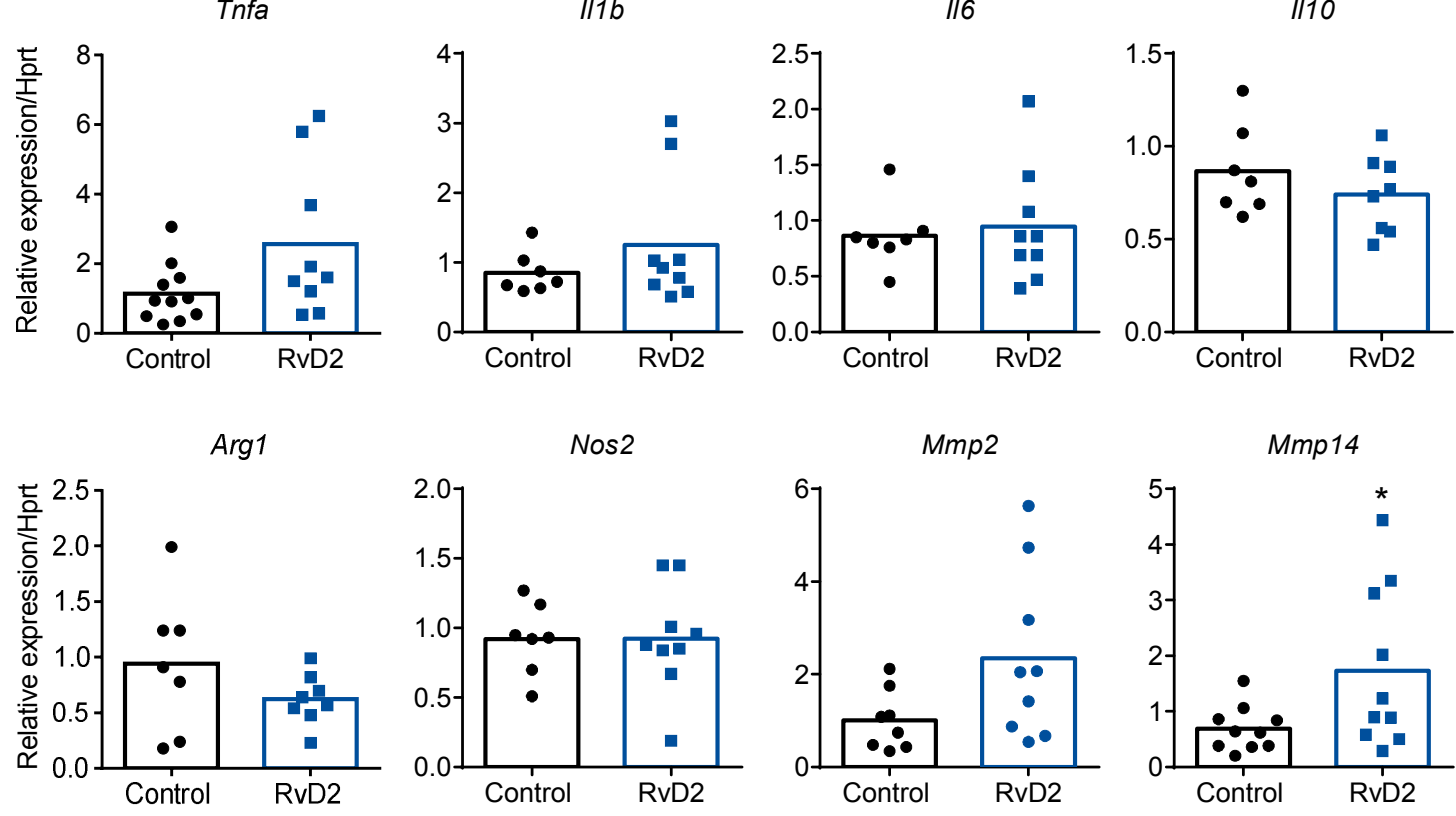

Cxcl12

Ptgs2

Alox 5

Alox15
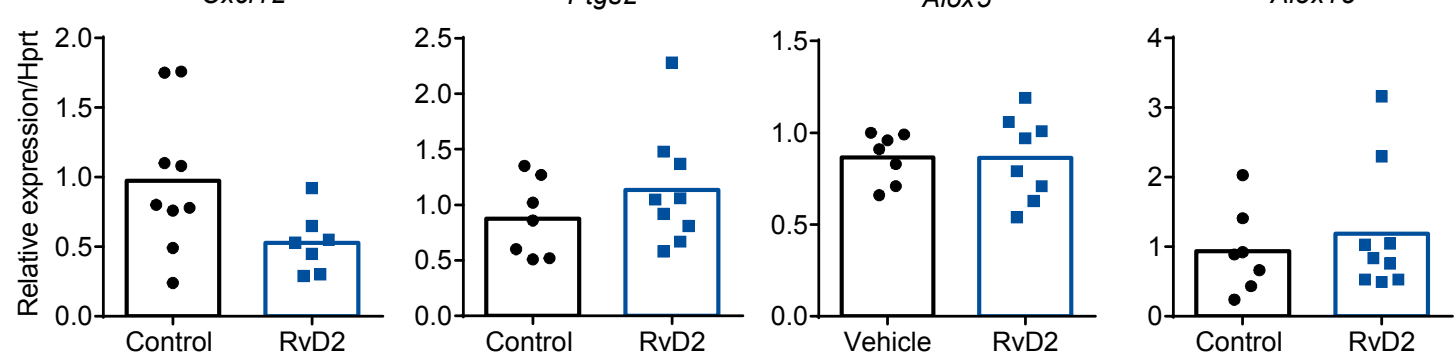
Figure 12: RvD2 stimulates endothelial migration but not proliferation. (A) RvD2 stimulates MS1 endothelial cell migration in a dose dependent manner. Representative scratch assay pictures indicate extent of migration. $n=6,{ }^{*} p<0.05$ (B) RvD2 does not stimulate MS1 endothelial cell proliferation by EdU, Hoescht or MTT proliferation assays. $n=9$, ${ }^{*} p<0.05$ 
Figure 11

A.
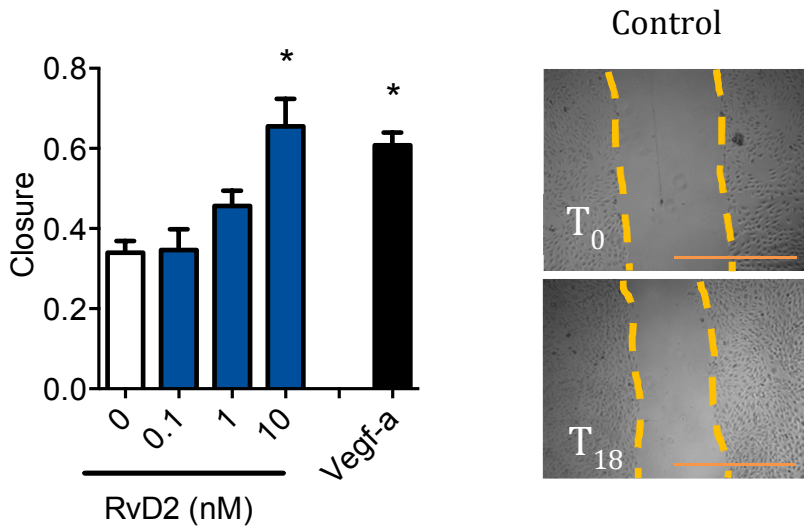

C.

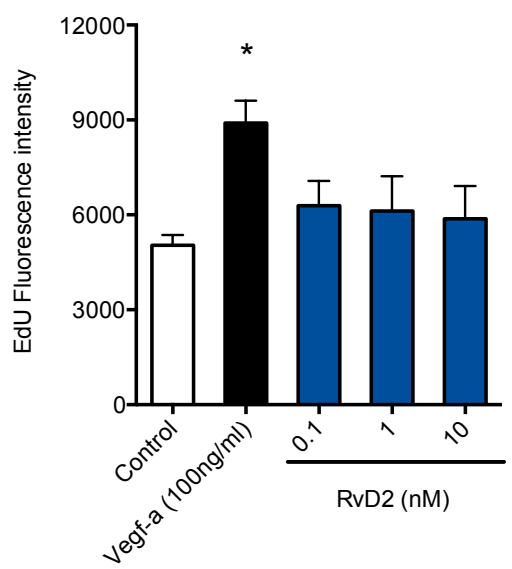

D.

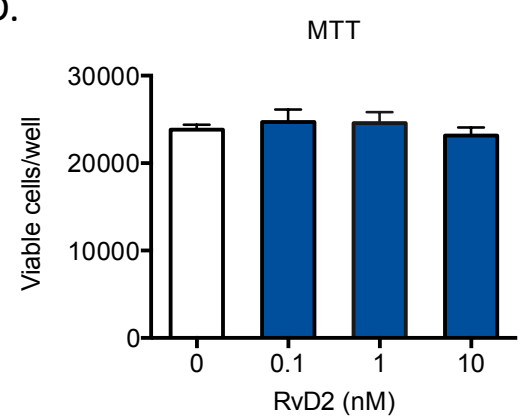

Control

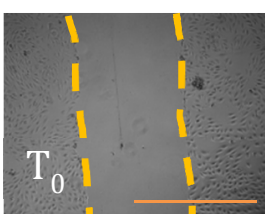

RvD2
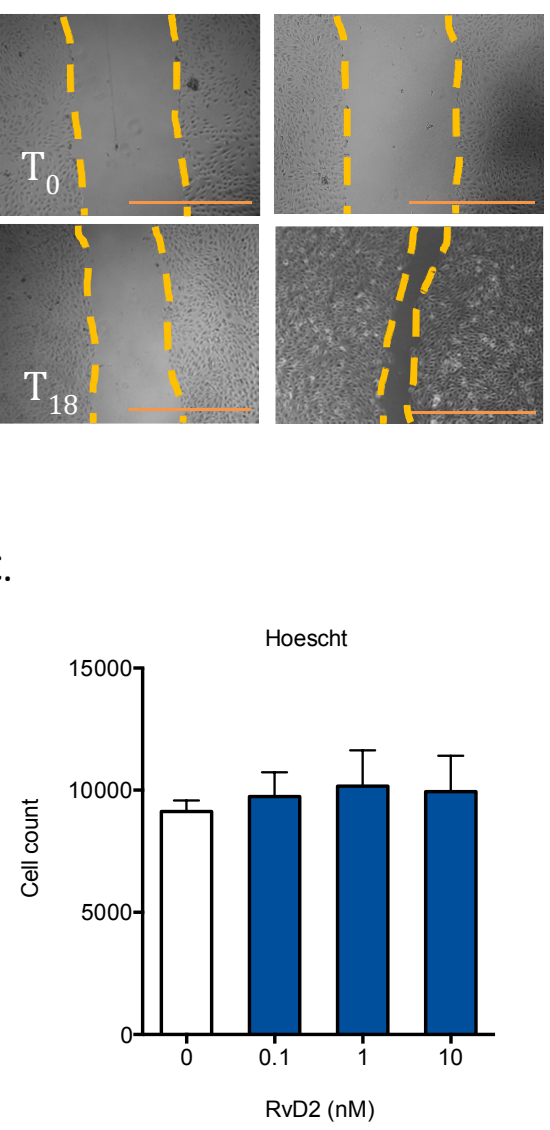
Figure 13: RvD2 does not stimulate angiogenesis. (A) RT-PCR of MS1 endothelial cells stimulated with 10nM of RvD1 for 4 hours. $n=3,{ }^{*} p<0.05$ (B) Representative photos of excised Matrigel plugs 7 days after implantation, and flow cytometry quantitation of plug-infiltrated endothelial cells. $n=$ $3,{ }^{*} p<0.05$ 
Figure 12

A.
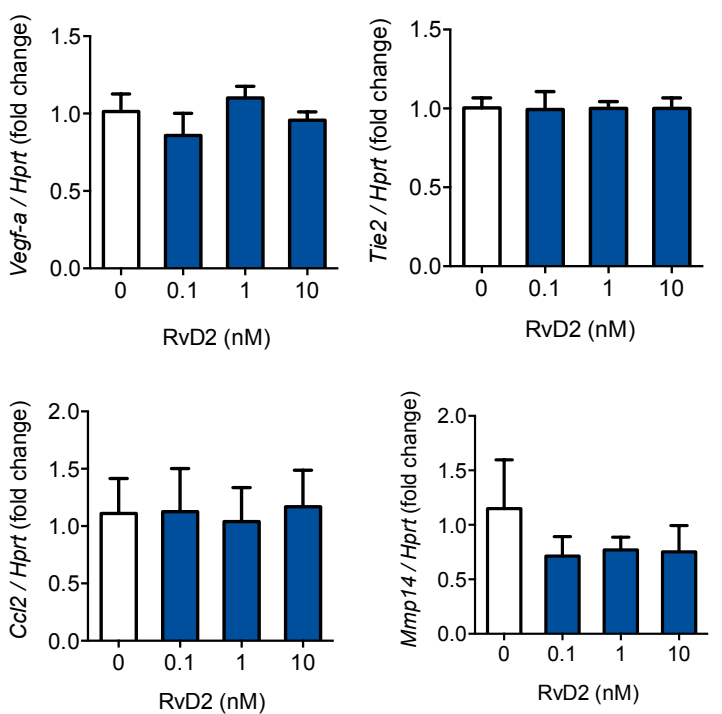

B.

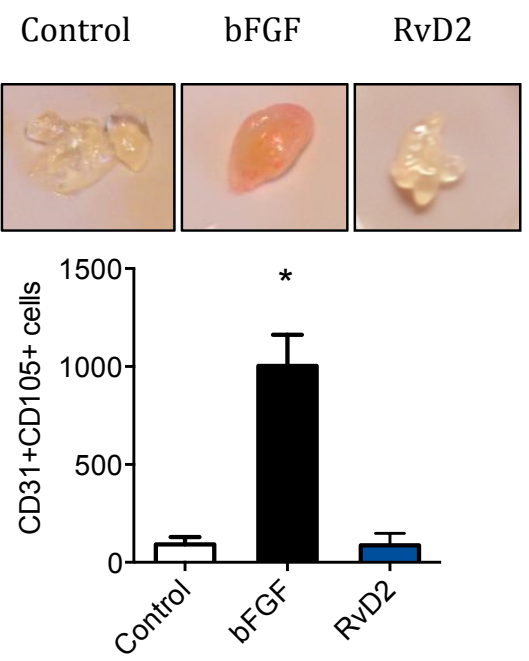


Figure 14: RvD2 stimulates migration through eNOS and Rac1. (A) Inhibition of MS1 endothelial migration by eNOS inhibitor L-NAME and Rac1 inhibitor NSC23766 in scratch assays. $n=6$, ${ }^{*} p<0.05$ (B) Representative blots of Rac1 activation assay. MS1 endothelial cells were stimulated with 10 nM of RvD2 for 30 min. $n=6,{ }^{*} p<0.05$ 
Figure 13

A.

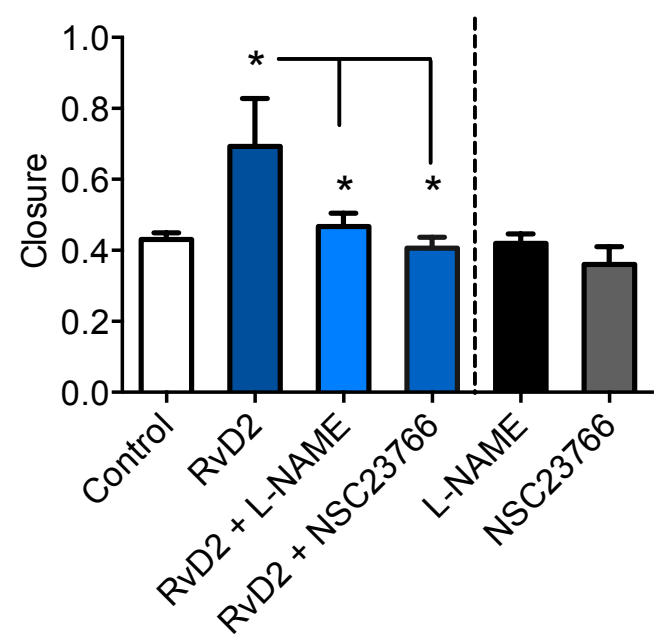

B.
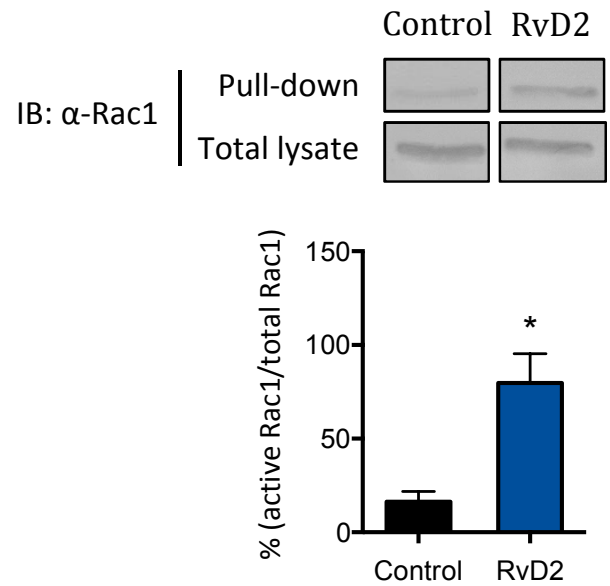


\section{Discussion}

The major findings of this study are that: RvD2 treatment modulates peripheral blood cytokine levels but not muscle leukocyte populations 3 days after HLI induction; RvD2 stimulates modest gene transcription in 3-day ischemic muscle but not gene transcription in isolated macrophages and endothelial cells; and RvD2 stimulates endothelial migration but not proliferation or angiogenesis. Taken together with our previous study demonstrating revascularization enhancement (Chapter 2), we conclude that RvD2 promotes revascularization by stimulating endothelial migration to enhance arteriogenesis, but not by stimulating angiogenesis.

RvD2 enhances arteriogenesis because it stimulated a robust increase in limb perfusion within the first week after femoral artery and vein occlusion. In our LDPI data, RvD2-treated mice showed increased ischemic limb perfusion. This increase was $475 \%$ from $8 \%$ of the non-ischemic $\operatorname{limb}$ to $46 \%$ by day 7 , but in vehicle-treated mice perfusion was increased only by $150 \%$ from $8 \%$ of the non-ischemic limb to $20 \%$. Between day 7 and 14 , RvD2-treated mice gained a further $40 \%$ increase in perfusion, while the gain in vehicle-treated mice was $48 \%$. Therefore the rate of recovery in the second week was very similar compared with the rate of recovery in the first week. This recovery differential is corroborated with our Microfil-microCT data. In the 3-day mild-HLI Microfil-microCT data, the vascular volume differential between RvD2 and vehicle treatments was $23 \%$. In the 7 -day severe-HLI data, the vascular volume differential was only $11 \%$. The potential acute role for RvD2 during revascularization has cascading effects on many of our subsequent cellular and molecular analyses. A robust arteriogenic response can effectively restore perfusion, reduce hypoxic stimulus, and dispense with a robust angiogenic response ${ }^{75}$. This is highlighted in the Microfil-microCT pictures. Despite an obvious focal interruption of blood flow, the immediate downstream femoral artery and vein sections are still perfused with Microfil. Clearly, collateral blood flow compensated for the focal occlusion. As a result, because arteriogenic perfusion 
recovery predominates immediately after occlusion, and our physiologic evidence supported an acute role for RvD2 during revascularization, we focused our subsequent analyses on mechanisms within the first week after HLI.

The 3 day post-HLI muscle cell population and muscle mRNA expression data do not show major changes. RvD2, and other resolvins, robustly decrease neutrophil infiltration in many inflammation models ${ }^{114}$. Although we found a trend towards a decrease in neutrophils with RvD2, the data was not statistically significant. This is most likely due to a lack of statistical power or measurement at a suboptimal time. Other leukocytes, including monocytes and macrophages, did not change either. However, unchanged cell numbers do not preclude stimulation of acute actions. We also interrogated gene expression in both the hamstring muscles and the gastrocnemius. We did not find any differences in the gastrocnemius (data not shown) and only modest changes in Vegf, Tnfa, Tie2 and Arg1. However, these gene transcription experiments were performed with the tissue, which contains a heterogenous mixture of cells. Differential gene transcription changes in different cells could potentially mute overall transcription changes. Also, mRNA expression changes may not directly correlate with acute physiologic and cellular actions; VEGF can be produced a priori and stored in ECM-bound granules ${ }^{155}$. These limitations prompted us to interrogate systemic soluble mediator changes and isolated cell stimulation.

To assess RvD2 and revascularization-induced changes in realtime, we also analyzed $3 \mathrm{~d}$ plasma with a multiplex ELISA of cytokines and chemokines. At this time, we found that there was a decrease to baseline in TNF- $\alpha$ levels and an increase above detection in IL-10 levels. These observations are consistent with the forward shifting of the inflammation and revascularization timecourse $^{43}$. TNF $\alpha$ is the prototypical inflammation-phase cytokine, and IL-10 is a hallmark resolution-phase cytokine ${ }^{14}$. Inflammation is an important initiating process of revascularization because it recruits leukocytes to the occlusion site to clean apoptotic and necrotic cell debris and 
digest extracellular matrix (ECM) to allow tissue remodeling during subsequent arteriogenesis and angiogenesis. The neutrophil is the predominant early-recruited inflammatory leukocyte during revascularization, but because it is also recruited during microbial infections, it is not discriminating like lymphocytes and will generate reactive oxygen species, produce ECM digesting elastases, and secrete inflammatory cytokines regardless of invading pathogens ${ }^{43}$. RvD2 has already been shown to be effective in attenuating neutrophil infiltration and reducing their cytokine secretion in other inflammatory models ${ }^{55}$. This immunomodulation limits collateral inflammation-produced damage and expedites the initiation of resolution, another important process of revascularization. During resolution, monocytes and macrophage secrete growth factors that stimulate arteriogenesis and angiogenesis and recruit mesenchymal cells to regenerate tissue ${ }^{86}$. The decrease in TNF- $\alpha$ and increase in IL-10 suggests that at day 3 postocclusion, RvD2-treated animals were tapering inflammation and initiating resolution earlier than vehicle-treated animals. Furthermore, Tnfa mRNA also decreased in the hamstring muscles at day 3, reaffirming our analysis.

Other significant changes in cytokines and chemokines were also observed. We found that IL-4 increased in RvD2-treated animals; it is the original stimulator of M2 macrophage polarization $^{81}$, and it increases $12 / 15$-lipoxygenase activity in macrophages ${ }^{156} . \mathrm{M} 2$ macrophage polarization is important during revascularization and wound healing, because M2-polarized macrophages secrete growth factors instead of inflammatory cytokines (a la M1 inflammatory macrophages) to promote revascularization and repair by endothelial cells, vascular smooth muscle cells and fibroblasts ${ }^{157}$. We concomitantly measured an increase in the transcription of murine M2 marker, Arg1, in the muscle, but no changes in other M2 markers (Mrc1, Retnla, Ym1, data not shown) or Alox15 (12/15-LOX gene, data not shown). It is important to emphasize that we only examined one time period during revascularization and therefore expression of other M2 
markers are likely time dependent. Additionally, there was also a puzzling lack of RvD2-stimulated change in CCL2. CCL2 is a major arteriogenic and angiogenic chemokine stimulated by arterial stretching and fluid shear stress to recruit monocytes and macrophages for promoting revascularization ${ }^{158}$. This is most likely due to low experiment $n$ and low statistical power as there is a promising trend in CCL2 decrease. Furthermore, this can be consistent with a robust RvD2arteriogenic response that decreases the need for additional revascularization-promoting mediators. This may also explain why GM-CSF, an arteriogenic cytokine, was robustly increased in vehicle-treated HLI mice but not in RvD2-treated mice. A randomized, double-blind, placebo controlled clinical trial has already proved the promotion of collateral growth by $\mathrm{GM}-\mathrm{CSF}^{159}$. Therefore the robust RvD2-stimulated revascularization response was sufficiently adequate to not require GM-CSF. Overall, blood cytokine and chemokine levels may not be entirely indicative of gradients at the actual injury site, and allosteric regulation between these soluble mediators can produce nonlinear effects that are difficult to reconcile given a single point of analysis. Therefore, given the paradoxical enhanced-revascularization dearth of mediator production in our data, we needed additional evidence to evaluate if mechanisms of RvD2 actions included stimulation of angiogenesis.

We evaluated the potential angiogenic mechanism of RvD2 action using an in vivo Matrigel plug angiogenesis assay and direct in vitro stimulation of isolated macrophages and endothelial cells with RvD2. The Matrigel plug assay is a robust method to interrogate angiogenicity: the plug provides an angiogenic environment only; the host mouse mobilizes the cells that ultimately vascularize the plug $^{153}$. Because RvD2-treated mice recover acutely, we may not have been able to assess accurately RvD2's angiogenicity in the HLI model. Using the Matrigel plug assay, which only measures angiogenesis, allowed us to focus our assessment. Furthermore, our utilization of a flow cytometer and a quadruple surface marker panel to enumerate Matrigel 
plug contents is rigorous and objective. That in comparison with the positive control bFGF, RvD2 did not stimulate any endothelial infiltration into the Matrigel plug, provides strong evidence against an angiogenic mode of action of RvD2.

We also performed additional RvD2-stimulated gene transcription analyses in isolated macrophages and endothelial cells. Our results were unremarkable, although there was an increase in Mmp14, or membrane type-1 metalloproteinase, which can promote tumor angiogenesis in a hypoxia independent manner ${ }^{160}$. No other angiogenic genes were increased however. Nevertheless, the in vitro characterization of RvD2 on monocytes and macrophages can be improved in the future. Monocytes and macrophages are highly plastic and therefore very heterogenous. We only examined the effects of RvD2 on one population of macrophages (peritoneal) and with limited forms of stimulation. To characterize the effects of RvD2 on monocytes and macrophages more rigorously work with more distinct populations and more stimulation methods will need to be performed.

Endothelial cell proliferation was also tested, via three different assays, and no mitogenicity was detected. Although endothelial proliferation is the primary component of angiogenesis, it can be stimulated by arteriogenesis, too. The non-mitogenic effect of RvD2, as evidenced by the proliferation studies and the Matrigel plug, suggests a more acute process. The RvD2-stimulated endothelial migration data supports this acute process. Endothelial migration can be stimulated mechanically by fluid shear stress (mechanotaxis), the same force that stimulates arteriogenesis ${ }^{154}$. Shear stress is the component of hemodynamic stress sensed by the luminal endothelium and transmitted into the component cells to activate an intricate network of migration-mediating GTPases. Specifically, shear stress induces microtubule elongation, which then activates Rac to promote actin polymerization and lamellipodia protrusion in the direction of flow. Concomitantly, RhoA becomes restricted to the back of the cell to generate the contraction 
required for rear detachment and migration. Additionally, shear stress can increase membrane fluidity and initiate signaling cascades from caveolae to activate enzymes such as eNOS. Hence, shear stress is a continuous mechanotactic stimulus for endothelial cells and contributes to cell migration during arteriogenesis. Our results, demonstrating activation of Rac1 by RvD2, and inhibition of RvD2-stimulated migration by Rac1 and eNOS inhibitors, further supports a migration-stimulating mechanism. Additionally, in other studies, inhibition of Rac1 or eNOS activity by gene knockout impaired revascularization after hind limb ischemia ${ }^{161-163}$. Therefore these two proteins and their signaling networks are necessary for revascularization, and because RvD2 enhancement of revascularization could result from stimulating these networks. Taken together with the in vivo HLI data, we conclude that RvD2 promotes arteriogenesis through the stimulation of endothelial migration.

Despite all the positive data, we did not provide causative evidence linking endothelial migration-stimulation and arteriogenic revascularization by RvD2 during HLI. This can be established in future interventional experiments. Inhibiting the RvD2 receptor specifically on endothelial cells and reducing revascularization to vehicle-treatment levels, would provide a causative link. Unfortunately the RvD2 receptor is currently unknown. Some evidence suggests it is a $\mathrm{Ga}_{\mathrm{i}}$-linked GPCR, pertussis toxin treatment is sufficient to inhibit HUVEC production of $\mathrm{NO}^{55}$. In the meantime, another endothelial-specific intervention can provide clues. Endothelial nitric oxide synthase is stimulated by fluid shear stress, participates in endothelial migration, and expressed specifically in endothelial cells ${ }^{161}$. Repeating the in vivo HLI studies with eNOS knockout mice would provide valuable causative insights. Additionally, we can attempt to bypass the more arteriogenic phase of revascularization and only begin RvD2 treatment 7 days after HLI surgery. This will produce an additional direct test of RvD2 effects on the more angiogenic phase of revascularization. Finally, even the vehicle-treated mice, at 8 weeks of age, recover robustly after 
HLI. Patients suffering from critical limb ischemia, and coronary artery disease, are proportionally older and possess other comorbidities ${ }^{90}$. Therefore testing RvD2 efficacy in older and diabetic mice would be an important translational investigation.

The isolated cellular experiments can be developed further as well. We did not investigate the role of VSMC in the HLI model or in in vitro assays. Some RvD2 actions on VSMC have been described in another study, but their conclusion was that RvD2 inhibited VSMC proliferation, migration, and inflammatory gene transcription ${ }^{151}$. This is paradoxical when compared with the endothelial cell $(E C)$ data presented in this study. However, the primary in vivo model in the VSMC study was neointimal hyperplasia following vascular injury. This is different from HLI model in this present study and therefore differences in results are likely context dependent. Furthermore, other vascular mediators, such as NO, can have paradoxical effects as well: NO is critical for revascularization, but it also inhibits VSMC proliferation ${ }^{164}$. Therefore additional studies, perhaps interrogating the interplay between VSMC and EC, are needed to reconcile these seemingly opposing actions.

In conclusion, we provide mechanistic evidence supporting the notion that RvD2 stimulates endothelial migration during arteriogenic revascularization after critical limb ischemia. This finding suggests that the endothelium is a primary target of RvD2 during blood perfusion recovery. We also provide evidence showing a proliferative and angiogenic role for RvD2. The causative link between stimulated migration and enhanced revascularization is missing and further experiments are needed to establish this connection more firmly in the future. 


\section{CHAPTER V}

\section{CONCLUDING DISCUSSION}

During the course of their lifetime, all organisms have to protect themselves from exogenous and endogenous insults that threaten their existence. For animals, the most common exogenous insults involve microbial pathogens and traumatic injury leading to physical wounding. Endogenous insults often result from autoimmune responses and genetic derangements, but also chronic diseases such as respiratory disease, cancer and cardiovascular and cerebrovascular disease. Often these diseases are accompanied by tissue ischemia. Collectively, mortality associated with tissue injury accounts for $50 \%$ of total mortality in current human societies ${ }^{117}$. Like other animals, humans have evolved a highly dynamic, but well-orchestrated inflammatory response to neutralize pathogens and heal wounds. An important aspect of this response is tissue revascularization. However, the shift to a more sedentary lifestyle and the increase in disease comorbidities has outpaced evolutionary adaptations and overwhelmed their capacity to recover from ischemic tissue damage. Therefore, therapies to minimize ischemic tissue injury or to promote tissue revascularization are urgently needed to diminish the global burden of disease.

Recent epidemiological studies of human populations suggest a link between reduction of cardiovascular mortality and the consumption of $\omega-3$ polyunsaturated fatty acids, which might be related to the beneficial effects on immunity ${ }^{103}$. Although a major function of the immune response is to kill invading pathogens, and remove dead tissue, recent immunovascular studies show that monocytes and macrophages recruited during the inflammatory response play an indispensable role in the revascularization process ${ }^{165}$. These cells are capable of using- $\omega-3$ fatty 
acids to generate pro-resolving lipid mediators that modulate or resolve the inflammatory response ${ }^{55}$. Taken together this evidence suggests a potential link between cells (monocytes and macrophages) that mediate revascularization- and the PUFAs they metabolize. The central hypothesis of my work was that pro-resolving lipid mediators produced by monocytes and macrophages mediate in part the enhancement of monocyte, macrophage and endothelial cellmediated arteriogenesis and angiogenesis during the revascularization component of ischemic wound healing.

These investigations began with a direct treatment study to determine if the proresolving lipid mediator, RvD2, can enhance revascularization using a mouse model of hind limb ischemia. We induced ischemia in the hind limb of mice by ligating the femoral artery and vein in a severe-HLI model and by ligating the saphenous artery and vein in a mild-HLI model. We treated mice with RvD2 (or saline) daily, starting one day after HLI surgery, and then we monitored the longitudinal recovery of blood perfusion in the severe-HLI model using a laser Doppler perfusion imaging system for 14 days. We also quantitated total limb vascular volume inferior to the ligation site by Microfil-microCT in severe-HLI mice at 7 days after surgery and mild-HLI mice at 3 days. In all three experiments, we measured a significant increase in RvD2treated limb perfusion, compared with the level of perfusion in saline-treated limb. Particularly noteworthy is that we began RvD2 treatment $24 \mathrm{~h}$ after HLI surgery. This protocol was to simulate clinical scenario in which treatment is almost always after injury. From these results we conclude that treatment with RvD2 enhances revascularization of ischemic wounds.

Having established that RvD2 has significant revascularization-enhancing effects in a hind limb ischemia model, we next examined the biosynthesis of RvD2 by monocytes involved in revascularization. To address this aim we first measured metabolites of $12 / 15$-lipoxygenase, the first enzyme in RvD2 biosynthesis in vivo, and found significant activity during revascularization. 
We then measured leukocyte populations in the mouse limbs, at the same revascularization time point, and found an increase in the critical revascularization-mediating Ly $6 \mathrm{C}^{\text {hi }} \mathrm{CCR} 2^{+}$inflammatory monocytes. Finally, we isolated these inflammatory monocytes from intact mouse spleens and determined that they robustly produced RvD2. From this we conclude that revascularizationmediating inflammatory monocytes are capable of generating the pro-resolving lipid mediator RvD2 from the $\omega-3$ fatty acid docosahexaenoic acid.

After confirming that monocytes can produce RvD2, we sought to elucidate the mechanism by which RvD2 promotes revascularization. For this, we measured RvD2-induced changes in ischemic muscle cell populations, mRNA expression, and peripheral blood cytokines and chemokines. We found a shift in the inflammation-resolution-revascularization timecourse but no major changes in cell populations. We also tested whether RvD2 directly modulated arteriogenic genes in isolated macrophages and endothelial cells and found few changes. Additionally, we found no evidence for a direct angiogenic effect of RvD2 in a Matrigel plug assay as well as endothelial proliferation assays. Nevertheless, we observed that RvD2-promoted endothelial migration in a Rac1 and eNOS-dependent manner. Taken together with results from other investigators demonstrating RvD2 stimulation of endothelial NO production, we concluded that our evidence was consistent with the notion that RvD2 stimulates endothelial migration to promote arteriogenesis during revascularization.

There are several notable limitations of our study. Despite the evidence presented, there is a lack of data showing that inflammatory monocytes synthesize RvD2 in vivo. Our data also do not establish a causative link between RvD2 stimulation of endothelial migration and enhanced arteriogenesis. Although we found also that $12 / 15$-LOX is active and inflammatory monocytes are increased during revascularization, and that monocytes can produce RvD2, we did not directly demonstrate the biosynthesis of RvD2 in the ischemic limb specifically by monocytes. Three 
approaches in future studies can address this issue by: 1) establishing a detailed timecourse of 12/15-lipoxygenase activity during the days following HLI in the limb muscle to measure lipid mediator production and identify RvD2 biosynthesis; 2) generating a mouse lacking 12/15-LOX expression specifically in inflammatory monocytes and demonstrating both a decrease in revascularization and ablation of RvD2 generation; and 3) transplanting inflammatory monocytes from 12/15-LOX knockout mice into HLI-operated wild type mice to see whether loss of this enzyme prevents enhancement of revascularization by monocytes. Also, even though we provide rigorous evidence supporting an arteriogenic role and migration-enhancing role for RvD2, we do not have experimental evidence to causally link these two mechanisms. Establishing this link would involve the identification of RvD2 receptor, and selective ablation of this gene in endothelial cells, and see whether this would eliminate RvD2-enhanced revascularization. Alternatively, or in addition, RvD2 stimulated signaling networks, such as eNOS and Rac1 could be inhibited to determine whether the pro-arteriogenic properties of RvD2 are lost. Addressing these two primary limitations of our study will significantly strengthen our hypothesis that that RvD2 promotes tissue revascularization after ischemia.

The primary conclusion of our study is that RvD2, synthesized by monocytes, increases arteriogenic revascularization. Cardiovascular benefits of $\omega-3$ fatty acids have been postulated for years, but there have been few mechanistic studies to uncover the signaling pathways that mediate the beneficial effects of these fatty acids ${ }^{107}$. Herein, we present physiologic and biochemical evidence of a specific mechanism by which endogenous $\omega-3$ fatty acid metabolites can ameliorate a specific cardiovascular injury. These findings address a major unmet clinical need. With the cardiovascular portion of the global burden of disease currently at $50 \%$ and trending upward, discovery of agents that promote revascularization is likely to have wide 
significance. Our identification of RvD2 as a novel revascularization agent is therefore ripe for clinical translation. 


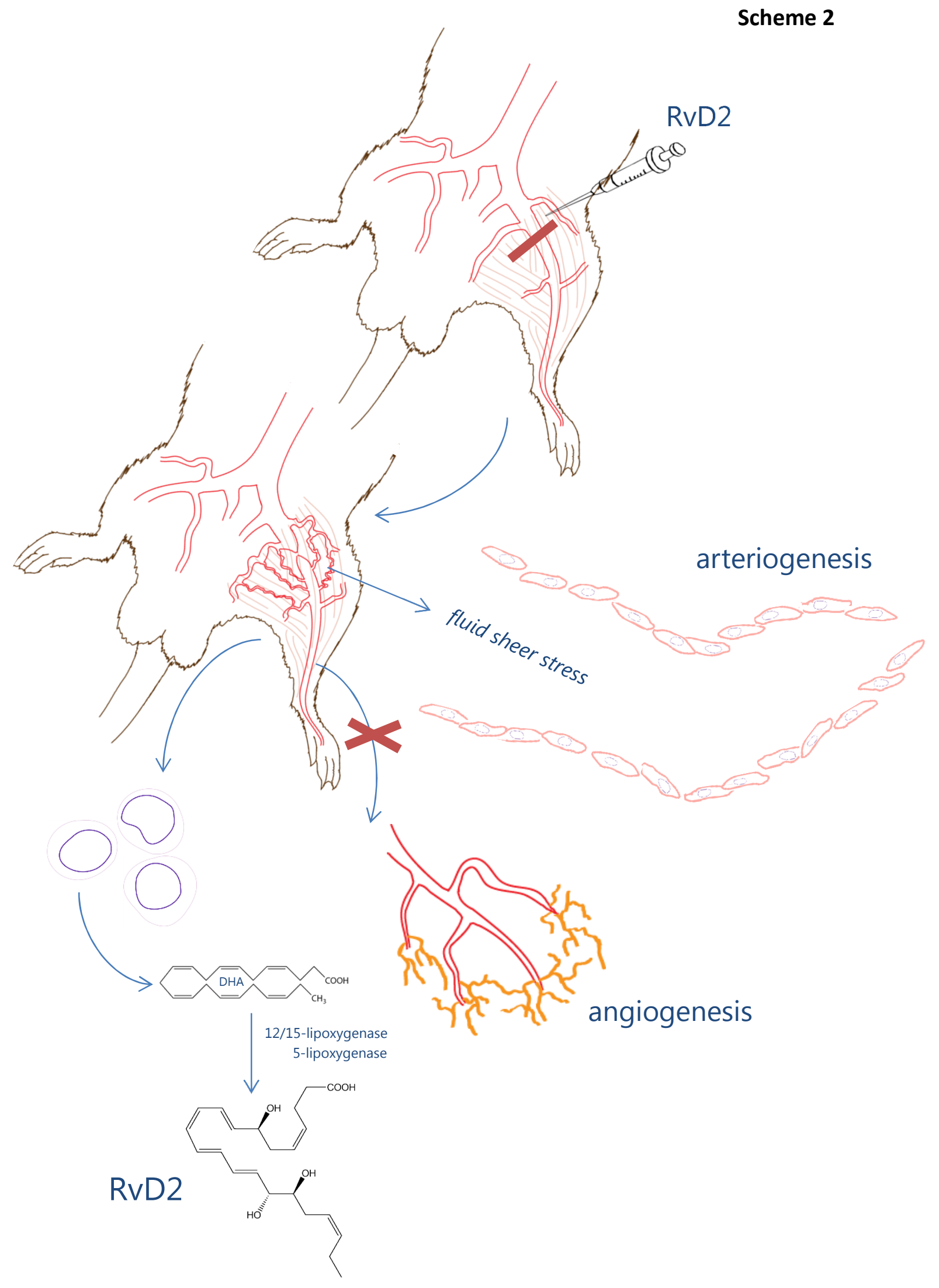




\section{REFERENCES}

1. Lenhart JS, Schroeder JW, Walsh BW, Simmons LA. DNA repair and genome maintenance in bacillus subtilis. Microbiology and molecular biology reviews : MMBR. 2012;76:530-564

2. Pfeifer K, Dorresteijn AW, Frobius AC. Activation of hox genes during caudal regeneration of the polychaete annelid platynereis dumerilii. Development genes and evolution. 2012;222:165-179

3. Laurie APoH, Chadwick, Chadwick LC. The modern nursery. A guide to plant propagation, culture and handling. New York: Macmillan Co.; 1931.

4. Driskell RR, Lichtenberger BM, Hoste E, Kretzschmar K, Simons BD, Charalambous M, Ferron SR, Herault Y, Pavlovic G, Ferguson-Smith AC, Watt FM. Distinct fibroblast lineages determine dermal architecture in skin development and repair. Nature. 2013;504:277-281

5. Jeon KW, Jeon MS. Cytoplasmic filaments and cellular wound healing in amoeba proteus. The Journal of cell biology. 1975;67:243-249

6. Ryan GB, Majno G. Inflammation. Kalamazoo, Mich.: Upjohn Co.; 1977.

7. Kumar V. Robbins and cotran's pathologic basis of disease. Philadelphia, Pa. ; London: Saunders; 2010.

8. Chapman HA, Jr. Role of enzyme receptors and inhibitors in regulating proteolytic activities of macrophages. Annals of the New York Academy of Sciences. 1991;624:87-96

9. Kawakami T, Obara W, Soma Y, Mizoguchi M. Palisading neutrophilic granulomatous dermatitis in a japanese patient with wegener's granulomatosis. The Journal of dermatology. 2005;32:487-492

10. Heidland A, Klassen A, Rutkowski $P$, Bahner $U$. The contribution of rudolf virchow to the concept of inflammation: What is still of importance? Journal of nephrology. 2006;19 Suppl 10:S102-109

11. Murphy K, Travers P, Walport M, Janeway C. Janeway's immunobiology. London: Garland Science ; London : Taylor \& Francis [distributor]; 2012.

12. Hall JE, Guyton ACPctTomp. Pocket companion to guyton \& hall textbook of medical physiology, eleventh edition. Philadelphia, Penn.: Elsevier Saunders; 2006.

13. Lewis SM, Wu GE. The origins of $v(d) j$ recombination. Cell. 1997;88:159-162

14. Serhan CN, Ward PA, Gilroy DW. Fundamentals of inflammation. Cambridge: Cambridge University Press; 2010.

15. Silverman GJ, Goodyear CS. Confounding b-cell defences: Lessons from a staphylococcal superantigen. Nat Rev Immunol. 2006;6:465-475

16. Ulloa L, Messmer D. High-mobility group box 1 (hmgb1) protein: Friend and foe. Cytokine \& growth factor reviews. 2006;17:189-201

17. Rock KL, Kono H. The inflammatory response to cell death. Annual review of pathology. 2008;3:99-126

18. Eltzschig HK, Carmeliet P. Hypoxia and inflammation. N Engl J Med. 2011;364:656-665

19. McEver RP. Rolling back neutrophil adhesion. Nat Immunol. 2010;11:282-284

20. Jack DL, Klein NJ, Turner MW. Mannose-binding lectin: Targeting the microbial world for complement attack and opsonophagocytosis. Immunological reviews. 2001;180:86-99 
21. Canton J, Neculai D, Grinstein S. Scavenger receptors in homeostasis and immunity. Nat Rev Immunol. 2013;13:621-634

22. Mosser DM. Receptors on phagocytic cells involved in microbial recognition. Immunology series. 1994;60:99-114

23. Ravichandran KS. Beginnings of a good apoptotic meal: The find-me and eat-me signaling pathways. Immunity. 2011;35:445-455

24. Beaven MA. Histamine (second of two parts). N Engl J Med. 1976;294:320-325

25. Ryan GB, Majno G. Acute inflammation. A review. Am J Pathol. 1977;86:183-276

26. Anstey ML, Rogers SM, Ott SR, Burrows M, Simpson SJ. Serotonin mediates behavioral gregarization underlying swarm formation in desert locusts. Science. 2009;323:627-630

27. Masters BS. Nitric oxide synthases: Why so complex? Annual review of nutrition. 1994;14:131-145

28. Bragt PC, Bansberg JI, Bonta IL. Antiinflammatory effects of free radical scavengers and antioxidants: Further support for proinflammatory roles of endogenous hydrogen peroxide and lipid peroxides. Inflammation. 1980;4:289-299

29. Serhan CN, Hamberg M, Samuelsson B. Lipoxins: Novel series of biologically active compounds formed from arachidonic acid in human leukocytes. Proc Natl Acad Sci U S A. 1984;81:5335-5339

30. Furuyashiki T, Narumiya S. Stress responses: The contribution of prostaglandin e(2) and its receptors. Nature reviews. Endocrinology. 2011;7:163-175

31. Vance DE, Vance JE. Biochemistry of lipids, lipoproteins and membranes (5th edn.). Amsterdam ; London: Elsevier; 2008.

32. Levy BD, Clish CB, Schmidt B, Gronert K, Serhan CN. Lipid mediator class switching during acute inflammation: Signals in resolution. Nat Immunol. 2001;2:612-619

33. Samuelsson B, Dahlen SE, Lindgren JA, Rouzer CA, Serhan CN. Leukotrienes and lipoxins: Structures, biosynthesis, and biological effects. Science. 1987;237:1171-1176

34. Weaver CT, Hatton RD, Mangan PR, Harrington LE. Il-17 family cytokines and the expanding diversity of effector $t$ cell lineages. Annual review of immunology. 2007;25:821852

35. Koch AE, Polverini PJ, Kunkel SL, Harlow LA, DiPietro LA, Elner VM, Elner SG, Strieter RM. Interleukin-8 as a macrophage-derived mediator of angiogenesis. Science. 1992;258:17981801

36. Mukaida N, Harada A, Matsushima K. Interleukin-8 (il-8) and monocyte chemotactic and activating factor (mcaf/mcp-1), chemokines essentially involved in inflammatory and immune reactions. Cytokine \& growth factor reviews. 1998;9:9-23

37. Salcedo R, Young HA, Ponce ML, Ward JM, Kleinman HK, Murphy WJ, Oppenheim JJ. Eotaxin (ccl11) induces in vivo angiogenic responses by human $\mathrm{ccr} 3+$ endothelial cells. $J$ Immunol. 2001;166:7571-7578

38. Suffee N, Hlawaty $H$, Meddahi-Pelle A, Maillard L, Louedec L, Haddad O, Martin L, Laguillier C, Richard B, Oudar O, Letourneur D, Charnaux N, Sutton A. Rantes/ccl5-induced pro-angiogenic effects depend on ccr1, ccr5 and glycosaminoglycans. Angiogenesis. 2012;15:727-744

39. White GE, Greaves DR. Fractalkine: A survivor's guide: Chemokines as antiapoptotic mediators. Arterioscler Thromb Vasc Biol. 2012;32:589-594

40. Kaplan JL, Porter RS, Szilagyi PG, Beers MH. The merck manual of medical information. New York: Pocket Books; 2007. 
41. Serhan CN, Brain SD, Buckley CD, Gilroy DW, Haslett C, O'Neill LA, Perretti M, Rossi AG, Wallace JL. Resolution of inflammation: State of the art, definitions and terms. FASEB J. 2007;21:325-332

42. Svensson $\mathrm{CI}$, Zattoni $\mathrm{M}$, Serhan $\mathrm{CN}$. Lipoxins and aspirin-triggered lipoxin inhibit inflammatory pain processing. J Exp Med. 2007;204:245-252

43. Serhan CN, Savill J. Resolution of inflammation: The beginning programs the end. Nat Immunol. 2005;6:1191-1197

44. Savill JS, Wyllie AH, Henson JE, Walport MJ, Henson PM, Haslett C. Macrophage phagocytosis of aging neutrophils in inflammation. Programmed cell death in the neutrophil leads to its recognition by macrophages. J Clin Invest. 1989;83:865-875

45. Swirski FK, Nahrendorf $M$, Etzrodt $M$, Wildgruber $M$, Cortez-Retamozo V, Panizzi $P$, Figueiredo JL, Kohler RH, Chudnovskiy A, Waterman P, Aikawa E, Mempel TR, Libby P, Weissleder $R$, Pittet MJ. Identification of splenic reservoir monocytes and their deployment to inflammatory sites. Science. 2009;325:612-616

46. Thorp E, Tabas I. Mechanisms and consequences of efferocytosis in advanced atherosclerosis. Journal of leukocyte biology. 2009;86:1089-1095

47. Savill J. Apoptosis in resolution of inflammation. Journal of leukocyte biology. 1997;61:375380

48. Kearley J, Barker JE, Robinson DS, Lloyd CM. Resolution of airway inflammation and hyperreactivity after in vivo transfer of $\mathrm{cd} 4+\mathrm{cd} 25+$ regulatory $\mathrm{t}$ cells is interleukin 10 dependent. J Exp Med. 2005;202:1539-1547

49. Fine A, Goldstein RH. The effect of transforming growth factor-beta on cell proliferation and collagen formation by lung fibroblasts. J Biol Chem. 1987;262:3897-3902

50. Dalli J, Norling LV, Renshaw D, Cooper D, Leung KY, Perretti M. Annexin 1 mediates the rapid anti-inflammatory effects of neutrophil-derived microparticles. Blood. 2008;112:2512-2519

51. Urbach V, Higgins G, Buchanan P, Ringholz F. The role of lipoxin a4 in cystic fibrosis lung disease. Computational and structural biotechnology journal. 2013;6:e201303018

52. Serhan CN. Resolution phase of inflammation: Novel endogenous anti-inflammatory and proresolving lipid mediators and pathways. Annual review of immunology. 2007;25:101137

53. Serhan CN, Clish CB, Brannon J, Colgan SP, Chiang N, Gronert K. Novel functional sets of lipid-derived mediators with antiinflammatory actions generated from omega-3 fatty acids via cyclooxygenase 2-nonsteroidal antiinflammatory drugs and transcellular processing. J Exp Med. 2000;192:1197-1204

54. Xu ZZ, Zhang L, Liu T, Park JY, Berta T, Yang R, Serhan CN, Ji RR. Resolvins rve1 and rvd1 attenuate inflammatory pain via central and peripheral actions. Nature medicine. 2010;16:592-597, 591p following 597

55. Spite $M$, Norling LV, Summers L, Yang R, Cooper D, Petasis NA, Flower RJ, Perretti M, Serhan $\mathrm{CN}$. Resolvin $\mathrm{d} 2$ is a potent regulator of leukocytes and controls microbial sepsis. Nature. 2009;461:1287-1291

56. Sunderkotter C, Steinbrink K, Goebeler M, Bhardwaj R, Sorg C. Macrophages and angiogenesis. Journal of leukocyte biology. 1994;55:410-422

57. Duffield JS, Lupher M, Thannickal VJ, Wynn TA. Host responses in tissue repair and fibrosis. Annual review of pathology. 2013;8:241-276 
58. Maxson S, Lopez EA, Yoo D, Danilkovitch-Miagkova A, Leroux MA. Concise review: Role of mesenchymal stem cells in wound repair. Stem cells translational medicine. 2012;1:142149

59. Gurtner GC, Werner S, Barrandon Y, Longaker MT. Wound repair and regeneration. Nature. 2008;453:314-321

60. Schurch W, Seemayer TA, Gabbiani G. The myofibroblast: A quarter century after its discovery. The American journal of surgical pathology. 1998;22:141-147

61. Yancopoulos GD, Klagsbrun M, Folkman J. Vasculogenesis, angiogenesis, and growth factors: Ephrins enter the fray at the border. Cell. 1998;93:661-664

62. Welm B, Mott J, Werb Z. Developmental biology: Vasculogenesis is a wreck without reck. Current biology: CB. 2002;12:R209-211

63. Goldie LC, Nix MK, Hirschi KK. Embryonic vasculogenesis and hematopoietic specification. Organogenesis. 2008;4:257-263

64. Murasawa S, Asahara T. Endothelial progenitor cells for vasculogenesis. Physiology (Bethesda). 2005;20:36-42

65. Gridley T. Notch signaling in vascular development and physiology. Development. 2007;134:2709-2718

66. Davis GE, Senger DR. Endothelial extracellular matrix: Biosynthesis, remodeling, and functions during vascular morphogenesis and neovessel stabilization. Circ Res. 2005;97:1093-1107

67. Robinson SD, Hodivala-Dilke KM. The role of beta3-integrins in tumor angiogenesis: Context is everything. Current opinion in cell biology. 2011;23:630-637

68. Carmeliet P, Collen D. Development and disease in proteinase-deficient mice: Role of the plasminogen, matrix metalloproteinase and coagulation system. Thrombosis research. 1998;91:255-285

69. Belperio JA, Keane MP, Arenberg DA, Addison CL, Ehlert JE, Burdick MD, Strieter RM. Cxc chemokines in angiogenesis. Journal of leukocyte biology. 2000;68:1-8

70. Carmeliet P. Angiogenesis in health and disease. Nature medicine. 2003;9:653-660

71. Heil M, Schaper W. Influence of mechanical, cellular, and molecular factors on collateral artery growth (arteriogenesis). Circ Res. 2004;95:449-458

72. Losordo DW, Dimmeler S. Therapeutic angiogenesis and vasculogenesis for ischemic disease: Part ii: Cell-based therapies. Circulation. 2004;109:2692-2697

73. Buschmann I, Schaper W. The pathophysiology of the collateral circulation (arteriogenesis). The Journal of pathology. 2000;190:338-342

74. Wang DL, Wung BS, Shyy YJ, Lin CF, Chao YJ, Usami S, Chien S. Mechanical strain induces monocyte chemotactic protein-1 gene expression in endothelial cells. Effects of mechanical strain on monocyte adhesion to endothelial cells. Circ Res. 1995;77:294-302

75. van Royen N, Piek JJ, Buschmann I, Hoefer I, Voskuil M, Schaper W. Stimulation of arteriogenesis; a new concept for the treatment of arterial occlusive disease. Cardiovascular research. 2001;49:543-553

76. Gordon S, Taylor PR. Monocyte and macrophage heterogeneity. Nat Rev Immunol. 2005;5:953-964

77. Yount L. A to $z$ of biologists. New York: Facts On File ; [London : Eurospan, distributor]; 2003.

78. Arnold L, Henry A, Poron F, Baba-Amer Y, van Rooijen N, Plonquet A, Gherardi RK, Chazaud B. Inflammatory monocytes recruited after skeletal muscle injury switch into antiinflammatory macrophages to support myogenesis. J Exp Med. 2007;204:1057-1069 
79. Geissmann F, Jung S, Littman DR. Blood monocytes consist of two principal subsets with distinct migratory properties. Immunity. 2003;19:71-82

80. Gordon S. Alternative activation of macrophages. Nat Rev Immunol. 2003;3:23-35

81. Stein $M$, Keshav $S$, Harris N, Gordon S. Interleukin 4 potently enhances murine macrophage mannose receptor activity: A marker of alternative immunologic macrophage activation. J Exp Med. 1992;176:287-292

82. Mosser DM, Edwards JP. Exploring the full spectrum of macrophage activation. Nat Rev Immunol. 2008;8:958-969

83. Munder M, Eichmann K, Modolell M. Alternative metabolic states in murine macrophages reflected by the nitric oxide synthase/arginase balance: Competitive regulation by $\mathrm{cd} 4+\mathrm{t}$ cells correlates with th1/th2 phenotype. J Immunol. 1998;160:5347-5354

84. Murray PJ, Wynn TA. Protective and pathogenic functions of macrophage subsets. Nat Rev Immunol. 2011;11:723-737

85. Raval Z, Losordo DW. Cell therapy of peripheral arterial disease: From experimental findings to clinical trials. Circ Res. 2013;112:1288-1302

86. Shireman PK. The chemokine system in arteriogenesis and hind limb ischemia. Journal of vascular surgery : official publication, the Society for Vascular Surgery [and] International Society for Cardiovascular Surgery, North American Chapter. 2007;45 Suppl A:A48-56

87. Powell RJ, Marston WA, Berceli SA, Guzman R, Henry TD, Longcore AT, Stern TP, Watling $S$, Bartel RL. Cellular therapy with ixmyelocel-t to treat critical limb ischemia: The randomized, double-blind, placebo-controlled restore-cli trial. Molecular therapy : the journal of the American Society of Gene Therapy. 2012;20:1280-1286

88. Keyes KT, Ye Y, Lin Y, Zhang C, Perez-Polo JR, Gjorstrup P, Birnbaum Y. Resolvin e1 protects the rat heart against reperfusion injury. Am J Physiol Heart Circ Physiol. 2010;299:H153-164

89. Duffield JS, Hong S, Vaidya VS, Lu Y, Fredman G, Serhan CN, Bonventre JV. Resolvin d series and protectin d1 mitigate acute kidney injury. J Immunol. 2006;177:5902-5911

90. Creager MA, Kaufman JA, Conte MS. Clinical practice. Acute limb ischemia. N Engl J Med. 2012;366:2198-2206

91. Chua RA, Arbiser JL. The role of angiogenesis in the pathogenesis of psoriasis. Autoimmunity. 2009;42:574-579

92. Singh NK, Kundumani-Sridharan V, Rao GN. 12/15-lipoxygenase gene knockout severely impairs ischemia-induced angiogenesis due to lack of rac1 farnesylation. Blood. 2011;118:5701-5712

93. Herxheimer H. Anti-histamine substances. Lancet. 1947;2:597

94. Kuzell WC. Nonsteroid anti-inflammatory agents. Annual review of pharmacology. 1968;8:357-376

95. Montague JW, Cidlowski JA. Glucocorticoid-induced death of immune cells: Mechanisms of action. Current topics in microbiology and immunology. 1995;200:51-65

96. Reichert JM. Marketed therapeutic antibodies compendium. mAbs. 2012;4:413-415

97. Hart FD, Huskisson EC. Non-steroidal anti-inflammatory drugs. Current status and rational therapeutic use. Drugs. 1984;27:232-255

98. Mukherjee D, Nissen SE, Topol EJ. Risk of cardiovascular events associated with selective cox-2 inhibitors. JAMA : the journal of the American Medical Association. 2001;286:954-959

99. Knizley $\mathrm{H}$, Jr., Mengel MC. Anti-inflammatory steroids. A review. JFMA, the Journal of the Florida Medical Association. Florida Medical Association. 1973;60:30-33 
100. Sinclair HM. Deficiency of essential fatty acids and atherosclerosis, etcetera. Lancet. 1956;270:381-383

101. Dyerberg J, Bang HO. Haemostatic function and platelet polyunsaturated fatty acids in eskimos. Lancet. 1979;2:433-435

102. Bang HO, Dyerberg J. Personal reflections on the incidence of ischaemic heart disease in oslo during the second world war. Acta medica Scandinavica. 1981;210:245-248

103. Marmot M, Elliott P. Coronary heart disease epidemiology : From aetiology to public health. Oxford: Oxford University Press; 2005.

104. Burr ML, Fehily AM, Gilbert JF, Rogers S, Holliday RM, Sweetnam PM, Elwood PC, Deadman NM. Effects of changes in fat, fish, and fibre intakes on death and myocardial reinfarction: Diet and reinfarction trial (dart). Lancet. 1989;2:757-761

105. Dietary supplementation with $n-3$ polyunsaturated fatty acids and vitamin e after myocardial infarction: Results of the gissi-prevenzione trial. Gruppo italiano per lo studio della sopravvivenza nell'infarto miocardico. Lancet. 1999;354:447-455

106. Tavazzi L, Maggioni AP, Marchioli R, Barlera S, Franzosi MG, Latini R, Lucci D, Nicolosi GL, Porcu $M$, Tognoni G. Effect of $n-3$ polyunsaturated fatty acids in patients with chronic heart failure (the gissi-hf trial): A randomised, double-blind, placebo-controlled trial. Lancet. 2008;372:1223-1230

107. Chowdhury R, Stevens S, Gorman D, Pan A, Warnakula S, Chowdhury S, Ward H, Johnson $\mathrm{L}$, Crowe $\mathrm{F}, \mathrm{Hu} \mathrm{FB}$, Franco OH. Association between fish consumption, long chain omega 3 fatty acids, and risk of cerebrovascular disease: Systematic review and meta-analysis. BMJ. 2012;345:e6698

108. Strasser T, Fischer S, Weber PC. Leukotriene b5 is formed in human neutrophils after dietary supplementation with icosapentaenoic acid. Proc Natl Acad Sci U S A. 1985;82:1540-1543

109. Wulff BC, Parent AE, Meleski MA, DiPietro LA, Schrementi ME, Wilgus TA. Mast cells contribute to scar formation during fetal wound healing. The Journal of investigative dermatology. 2012;132:458-465

110. Kang JX, Wang J, Wu L, Kang ZB. Transgenic mice: Fat-1 mice convert n-6 to n-3 fatty acids. Nature. 2004;427:504

111. Serhan CN, Hong S, Gronert K, Colgan SP, Devchand PR, Mirick G, Moussignac RL. Resolvins: A family of bioactive products of omega-3 fatty acid transformation circuits initiated by aspirin treatment that counter proinflammation signals. J Exp Med. 2002;196:1025-1037

112. Arita M, Bianchini F, Aliberti J, Sher A, Chiang N, Hong S, Yang R, Petasis NA, Serhan CN. Stereochemical assignment, antiinflammatory properties, and receptor for the omega-3 lipid mediator resolvin e1. J Exp Med. 2005;201:713-722

113. Krishnamoorthy $\mathrm{S}$, Recchiuti A, Chiang N, Yacoubian S, Lee $\mathrm{CH}$, Yang R, Petasis NA, Serhan $\mathrm{CN}$. Resolvin $\mathrm{d} 1$ binds human phagocytes with evidence for proresolving receptors. Proc Natl Acad Sci U S A. 2010;107:1660-1665

114. Zhang MJ, Spite M. Resolvins: Anti-inflammatory and proresolving mediators derived from omega-3 polyunsaturated fatty acids. Annual review of nutrition. 2012;32:203-227

115. Hellmann J, Tang Y, Kosuri M, Bhatnagar A, Spite M. Resolvin d1 decreases adipose tissue macrophage accumulation and improves insulin sensitivity in obese-diabetic mice. FASEB J. 2011;25:2399-2407 
116. Hellmann J, Zhang MJ, Tang Y, Rane M, Bhatnagar A, Spite M. Increased saturated fatty acids in obesity alter resolution of inflammation in part by stimulating prostaglandin production. J Immunol. 2013;191:1383-1392

117. Lozano R, Naghavi M, Foreman K, Lim S, Shibuya K, Aboyans V, Abraham J, Adair T, Aggarwal R, Ahn SY, Alvarado M, Anderson HR, Anderson LM, Andrews KG, Atkinson C, Baddour LM, Barker-Collo S, Bartels DH, Bell ML, Benjamin EJ, Bennett D, Bhalla K, Bikbov B, Bin Abdulhak A, Birbeck G, Blyth F, Bolliger I, Boufous S, Bucello C, Burch M, Burney P, Carapetis J, Chen H, Chou D, Chugh SS, Coffeng LE, Colan SD, Colquhoun S, Colson KE, Condon J, Connor MD, Cooper LT, Corriere M, Cortinovis M, de Vaccaro KC, Couser W, Cowie BC, Criqui MH, Cross M, Dabhadkar KC, Dahodwala N, De Leo D, Degenhardt L, Delossantos A, Denenberg J, Des Jarlais DC, Dharmaratne SD, Dorsey ER, Driscoll T, Duber $H$, Ebel $B$, Erwin PJ, Espindola P, Ezzati M, Feigin V, Flaxman AD, Forouzanfar $M H$, Fowkes FG, Franklin R, Fransen M, Freeman MK, Gabriel SE, Gakidou E, Gaspari F, Gillum RF, Gonzalez-Medina D, Halasa YA, Haring D, Harrison JE, Havmoeller R, Hay RJ, Hoen B, Hotez PJ, Hoy D, Jacobsen KH, James SL, Jasrasaria R, Jayaraman S, Johns N, Karthikeyan G, Kassebaum N, Keren A, Khoo JP, Knowlton LM, Kobusingye O, Koranteng $A$, Krishnamurthi R, Lipnick M, Lipshultz SE, Ohno SL, Mabweijano J, MacIntyre MF, Mallinger L, March L, Marks GB, Marks R, Matsumori A, Matzopoulos R, Mayosi BM, McAnulty JH, McDermott MM, McGrath J, Mensah GA, Merriman TR, Michaud C, Miller M, Miller TR, Mock C, Mocumbi AO, Mokdad AA, Moran A, Mulholland K, Nair MN, Naldi L, Narayan KM, Nasseri K, Norman P, O'Donnell M, Omer SB, Ortblad K, Osborne R, Ozgediz D, Pahari B, Pandian JD, Rivero AP, Padilla RP, Perez-Ruiz F, Perico N, Phillips D, Pierce K, Pope CA, 3rd, Porrini E, Pourmalek F, Raju M, Ranganathan D, Rehm JT, Rein DB, Remuzzi G, Rivara FP, Roberts T, De Leon FR, Rosenfeld LC, Rushton L, Sacco RL, Salomon JA, Sampson U, Sanman E, Schwebel DC, Segui-Gomez M, Shepard DS, Singh D, Singleton J, Sliwa K, Smith E, Steer A, Taylor JA, Thomas B, Tleyjeh IM, Towbin JA, Truelsen T, Undurraga EA, Venketasubramanian N, Vijayakumar L, Vos T, Wagner GR, Wang M, Wang W, Watt $K$, Weinstock MA, Weintraub R, Wilkinson JD, Woolf AD, Wulf $S$, Yeh PH, Yip P, Zabetian A, Zheng ZJ, Lopez AD, Murray CJ, AlMazroa MA, Memish ZA. Global and regional mortality from 235 causes of death for 20 age groups in 1990 and 2010: A systematic analysis for the global burden of disease study 2010. Lancet. 2012;380:2095-2128

118. Wennberg PW. Approach to the patient with peripheral arterial disease. Circulation. 2013;128:2241-2250

119. Limbourg A, Korff T, Napp LC, Schaper W, Drexler H, Limbourg FP. Evaluation of postnatal arteriogenesis and angiogenesis in a mouse model of hind-limb ischemia. Nature protocols. 2009;4:1737-1746

120. $W \backslash K, \backslash \# 551$, rdell, Anderson $C$, Andersson T. Visualization of the microcirculation by laser doppler perfusion imaging. SIGBIO Newsl. 1995;15:9-11

121. Duvall CL, Taylor WR, Weiss D, Guldberg RE. Quantitative microcomputed tomography analysis of collateral vessel development after ischemic injury. Am J Physiol Heart Circ Physiol. 2004;287:H302-310

122. Ciciliot S, Schiaffino S. Regeneration of mammalian skeletal muscle. Basic mechanisms and clinical implications. Current pharmaceutical design. 2010;16:906-914

123. Hur J, Yoon $\mathrm{CH}$, Kim HS, Choi JH, Kang HJ, Hwang KK, Oh BH, Lee MM, Park YB. Characterization of two types of endothelial progenitor cells and their different contributions to neovasculogenesis. Arterioscler Thromb Vasc Biol. 2004;24:288-293 
124. Makarevich P, Tsokolaeva Z, Shevelev A, Rybalkin I, Shevchenko E, Beloglazova I, Vlasik T, Tkachuk V, Parfyonova Y. Combined transfer of human vegf165 and hgf genes renders potent angiogenic effect in ischemic skeletal muscle. PLoS One. 2012;7:e38776

125. He Y, Luo Y, Tang S, Rajantie I, Salven P, Heil M, Zhang R, Luo D, Li X, Chi H, Yu J, Carmeliet P, Schaper W, Sinusas AJ, Sessa WC, Alitalo K, Min W. Critical function of bmx/etk in ischemia-mediated arteriogenesis and angiogenesis. J Clin Invest. 2006;116:2344-2355

126. Bondke A, Hillmeister P, Buschmann IR. Exact assessment of perfusion and collateral vessel proliferation in small animal models. Circ Res. 2007;100:e82-83

127. Zagorchev L, Mulligan-Kehoe MJ. Molecular imaging of vessels in mouse models of disease. European journal of radiology. 2009;70:305-311

128. Kochi T, Imai Y, Takeda A, Watanabe Y, Mori S, Tachi M, Kodama T. Characterization of the arterial anatomy of the murine hindlimb: Functional role in the design and understanding of ischemia models. PLoS One. 2013;8:e84047

129. Entabi F, Albadawi $H$, Stone DH, Sroufe R, Conrad MF, Watkins MT. Hind limb ischemiareperfusion in the leptin receptor deficient $(\mathrm{db} / \mathrm{db})$ mouse. The Journal of surgical research. 2007;139:97-105

130. Tomita-Yamaguchi M, Babich JF, Baker RC, Santoro TJ. Incorporation, distribution, and turnover of arachidonic acid within membrane phospholipids of b220 $+\mathrm{t}$ cells from autoimmune-prone mrl-Ipr/lpr mice. J Exp Med. 1990;171:787-800

131. Murakami M, Taketomi Y, Miki Y, Sato H, Hirabayashi T, Yamamoto K. Recent progress in phospholipase a(2) research: From cells to animals to humans. Progress in lipid research. 2011;50:152-192

132. Ghosh M, Tucker DE, Burchett SA, Leslie CC. Properties of the group iv phospholipase a2 family. Progress in lipid research. 2006;45:487-510

133. Marks F, Fu \rstenberger G. Prostaglandins, leukotrienes, and other eicosanoids : From biogenesis to clinical applications. Weinheim ; Cambridge: Wiley-VCH; 1999.

134. Hamberg M, Samuelsson B. On the mechanism of the biosynthesis of prostaglandins e-1 and f-1-alpha. J Biol Chem. 1967;242:5336-5343

135. McGee JE, Fitzpatrick FA. Erythrocyte-neutrophil interactions: Formation of leukotriene b4 by transcellular biosynthesis. Proc Natl Acad Sci U S A. 1986;83:1349-1353

136. Sun $D$, Funk CD. Disruption of 12/15-lipoxygenase expression in peritoneal macrophages. Enhanced utilization of the 5-lipoxygenase pathway and diminished oxidation of low density lipoprotein. J Biol Chem. 1996;271:24055-24062

137. Dalli J, Serhan CN. Specific lipid mediator signatures of human phagocytes: Microparticles stimulate macrophage efferocytosis and pro-resolving mediators. Blood. 2012;120:e60-72

138. Dobrian AD, Lieb DC, Cole BK, Taylor-Fishwick DA, Chakrabarti SK, Nadler JL. Functional and pathological roles of the 12- and 15-lipoxygenases. Progress in lipid research. 2011;50:115-131

139. Conrad DJ. The arachidonate 12/15 lipoxygenases. A review of tissue expression and biologic function. Clinical reviews in allergy \& immunology. 1999;17:71-89

140. Heil M, Ziegelhoeffer T, Wagner S, Fernandez B, Helisch A, Martin S, Tribulova S, Kuziel WA, Bachmann G, Schaper W. Collateral artery growth (arteriogenesis) after experimental arterial occlusion is impaired in mice lacking cc-chemokine receptor-2. Circ Res. 2004;94:671-677

141. Bergmann CE, Hoefer IE, Meder B, Roth H, van Royen N, Breit SM, Jost MM, Aharinejad S, Hartmann S, Buschmann IR. Arteriogenesis depends on circulating monocytes and 
macrophage accumulation and is severely depressed in op/op mice. Journal of leukocyte biology. 2006;80:59-65

142. Heil M, Ziegelhoeffer T, Pipp F, Kostin S, Martin S, Clauss M, Schaper W. Blood monocyte concentration is critical for enhancement of collateral artery growth. Am J Physiol Heart Circ Physiol. 2002;283:H2411-2419

143. Voskuil M, Hoefer IE, van Royen N, Hua J, de Graaf S, Bode C, Buschmann IR, Piek JJ. Abnormal monocyte recruitment and collateral artery formation in monocyte chemoattractant protein-1 deficient mice. Vasc Med. 2004;9:287-292

144. Capoccia BJ, Gregory AD, Link DC. Recruitment of the inflammatory subset of monocytes to sites of ischemia induces angiogenesis in a monocyte chemoattractant protein-1dependent fashion. Journal of leukocyte biology. 2008;84:760-768

145. Nichols RC, Vanderhoek JY. 5-hydroxyeicosanoids selectively stimulate the human neutrophil 15-lipoxygenase to use endogenous substrate. J Exp Med. 1990;171:367-375

146. Facciabene A, Motz GT, Coukos G. T-regulatory cells: Key players in tumor immune escape and angiogenesis. Cancer research. 2012;72:2162-2171

147. Tongers J, Roncalli JG, Losordo DW. Role of endothelial progenitor cells during ischemiainduced vasculogenesis and collateral formation. Microvascular research. 2010;79:200-206

148. Krenning G, Zeisberg EM, Kalluri R. The origin of fibroblasts and mechanism of cardiac fibrosis. Journal of cellular physiology. 2010;225:631-637

149. Kasuga K, Yang R, Porter TF, Agrawal N, Petasis NA, Irimia D, Toner M, Serhan CN. Rapid appearance of resolvin precursors in inflammatory exudates: Novel mechanisms in resolution. J Immunol. 2008;181:8677-8687

150. Schaper W, Scholz D. Factors regulating arteriogenesis. Arterioscler Thromb Vasc Biol. 2003;23:1143-1151

151. Miyahara T, Runge S, Chatterjee A, Chen M, Mottola G, Fitzgerald JM, Serhan CN, Conte MS. D-series resolvin attenuates vascular smooth muscle cell activation and neointimal hyperplasia following vascular injury. FASEB J. 2013;27:2220-2232

152. Bohr S, Patel SJ, Sarin D, Irimia D, Yarmush ML, Berthiaume F. Resolvin d2 prevents secondary thrombosis and necrosis in a mouse burn wound model. Wound repair and regeneration : official publication of the Wound Healing Society [and] the European Tissue Repair Society. 2013;21:35-43

153. Akhtar N, Dickerson EB, Auerbach R. The sponge/matrigel angiogenesis assay. Angiogenesis. 2002;5:75-80

154. Lamalice L, Le Boeuf F, Huot J. Endothelial cell migration during angiogenesis. Circ Res. 2007;100:782-794

155. Hutchings $\mathrm{H}$, Ortega $\mathrm{N}$, Plouet J. Extracellular matrix-bound vascular endothelial growth factor promotes endothelial cell adhesion, migration, and survival through integrin ligation. FASEB J. 2003;17:1520-1522

156. Heydeck D, Thomas L, Schnurr K, Trebus F, Thierfelder WE, Ihle JN, Kuhn H. Interleukin-4 and -13 induce upregulation of the murine macrophage 12/15-lipoxygenase activity: Evidence for the involvement of transcription factor stat6. Blood. 1998;92:2503-2510

157. Novak ML, Koh TJ. Macrophage phenotypes during tissue repair. Journal of leukocyte biology. 2013;93:875-881

158. Ito WD, Arras M, Winkler B, Scholz D, Schaper J, Schaper W. Monocyte chemotactic protein-1 increases collateral and peripheral conductance after femoral artery occlusion. Circ Res. 1997;80:829-837 
159. Seiler C, Pohl T, Wustmann K, Hutter D, Nicolet PA, Windecker S, Eberli FR, Meier B. Promotion of collateral growth by granulocyte-macrophage colony-stimulating factor in patients with coronary artery disease: A randomized, double-blind, placebo-controlled study. Circulation. 2001;104:2012-2017

160. Sounni NE, Devy L, Hajitou A, Frankenne F, Munaut C, Gilles C, Deroanne C, Thompson EW, Foidart JM, Noel A. Mt1-mmp expression promotes tumor growth and angiogenesis through an up-regulation of vascular endothelial growth factor expression. FASEB J. 2002;16:555-564

161. Sawada N, Salomone S, Kim HH, Kwiatkowski DJ, Liao JK. Regulation of endothelial nitric oxide synthase and postnatal angiogenesis by rac1. Circ Res. 2008;103:360-368

162. MacLauchlan S, Yu J, Parrish M, Asoulin TA, Schleicher M, Krady MM, Zeng J, Huang PL, Sessa WC, Kyriakides TR. Endothelial nitric oxide synthase controls the expression of the angiogenesis inhibitor thrombospondin 2. Proc Natl Acad Sci U S A. 2011;108:E1137-1145

163. Yu J, deMuinck ED, Zhuang Z, Drinane $M$, Kauser K, Rubanyi GM, Qian HS, Murata T, Escalante B, Sessa WC. Endothelial nitric oxide synthase is critical for ischemic remodeling, mural cell recruitment, and blood flow reserve. Proc Natl Acad Sci U S A. 2005;102:1099911004

164. Jeremy JY, Rowe D, Emsley AM, Newby AC. Nitric oxide and the proliferation of vascular smooth muscle cells. Cardiovascular research. 1999;43:580-594

165. Godwin JW, Pinto AR, Rosenthal NA. Macrophages are required for adult salamander limb regeneration. Proc Natl Acad Sci U S A. 2013;110:9415-9420 


\section{Education:}

$2004-2008$

$2009-2016$

\section{Employment:}

$\begin{array}{ll}2006-\text { Present } & \text { Research Assistant - Department of Anesthesiology at UCLA } \\ & \text { Advisor: Thomas Vondriska } \\ & \text { Research Assistant - Pasarow Mass Spectrometry Lab at UCLA } \\ & \text { Advisor: Kym Faull } \\ 2004-2006 & \text { Research Assistant - Department of Physiology at UCLA } \\ & \text { Advisor: Peipei Ping } \\ 2003-2005 & \text { Laboratory Assistant \& IT Technician - GenWay Biotech Inc. } \\ & \text { Supervisor: Robert Gans }\end{array}$

Awards and Honors:

$\begin{array}{ll}2007 & \text { Dean's Prize, Science Poster Day at (UCLA) } \\ 2007-2008 & \text { Undergraduate Research and Teaching Scholarship (UCLA) } \\ 2007 & \text { Undergraduate Research Center - Travel Award 2007 (UCLA) } \\ 2008 & \text { Dept. of Anesthesiology - Excellence in Science Oral Presentation Award (UCLA) } \\ 2009 & \text { Robert E. Brennan Scholarship (UofL) } \\ 2010 & \text { Salome E Kerr Scholarship (UofL) }\end{array}$

\section{Full Length Publications:}

1. Tang Y, Zhang MJ, Hellmann J, Kosuri M, Bhatnagar A, Spite M. Proresolution therapy for the treatment of delayed healing of diabetic wounds. Diabetes. 2013;62:618-627

2. Hellmann J, Zhang MJ, Tang $Y$, Rane $M$, Bhatnagar $A$, Spite $M$. Increased saturated fatty acids in obesity alter resolution of inflammation in part by stimulating prostaglandin production. J Immunol. 2013;191:1383-1392

3. Zhang MJ, Spite M. Resolvins: Anti-inflammatory and proresolving mediators derived from omega-3 polyunsaturated fatty acids. Annual review of nutrition. 2012;32:203-227 
4. Li Y, Franklin S, Zhang MJ, Vondriska TM. Highly efficient purification of protein complexes from mammalian cells using a novel streptavidin-binding peptide and hexahistidine tandem tag system: Application to bruton's tyrosine kinase. Protein science : a publication of the Protein Society. 2011;20:140-149

5. Franklin S, Zhang MJ, Chen H, Paulsson AK, Mitchell-Jordan SA, Li Y, Ping P, Vondriska TM. Specialized compartments of cardiac nuclei exhibit distinct proteomic anatomy. Mol Cell Proteomics. 2011;10:M110 000703

6. Zhang MJ, Franklin S, Li Y, Wang S, Ru X, Mitchell-Jordan SA, Mano H, Stefani E, Ping P, Vondriska TM. Stress signaling by tec tyrosine kinase in the ischemic myocardium. Am J Physiol Heart Circ Physiol. 2010;299:H713-722

7. Mitchell-Jordan SA, Holopainen T, Ren S, Wang S, Warburton S, Zhang MJ, Alitalo K, Wang Y, Vondriska TM. Loss of bmx nonreceptor tyrosine kinase prevents pressure overload-induced cardiac hypertrophy. Circ Res. 2008;103:1359-1362 